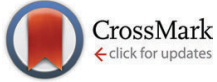

Cite this: Phys. Chem. Chem. Phys., 2015, 17, 29421

\section{Quantum theory of concerted electronic and nuclear fluxes associated with adiabatic intramolecular processes}

\author{
Timm Bredtmann, ${ }^{\text {ab }}$ Dennis J. Diestler, ${ }^{\text {cd }}$ Si-Dian Li, ${ }^{e}$ Jörn Manz, ${ }^{\text {acf }}$ \\ Jhon Fredy Pérez-Torres, ${ }^{c}$ Wen-Juan Tian, ${ }^{e}$ Yan-Bo Wu, ${ }^{e}$ Yonggang Yang ${ }^{\text {af }}$ and \\ Hua-Jin Zhai
}

\begin{abstract}
An elementary molecular process can be characterized by the flow of particles (i.e., electrons and nuclei) that compose the system. The flow, in turn, is quantitatively described by the flux (i.e., the time-sequence of maps of the rate of flow of particles though specified surfaces of observation) or, in more detail, by the flux density. The quantum theory of concerted electronic and nuclear fluxes (CENFs) associated with electronically adiabatic intramolecular processes is presented. In particular, it is emphasized how the electronic continuity equation can be employed to circumvent the failure of the Born-Oppenheimer approximation, which always predicts a vanishing electronic flux density (EFD). It is also shown that all CENFs accompanying coherent tunnelling between equivalent "reactant" and "product" configurations of isolated molecules are synchronous. The theory is applied to three systems of increasing complexity. The first application is to vibrating, aligned $\mathrm{H}_{2}{ }^{+}\left({ }^{2} \Sigma_{\mathrm{g}}{ }^{+}\right.$), or vibrating and dissociating $\mathrm{H}_{2}{ }^{+}\left({ }^{2} \Sigma_{\mathrm{g}}{ }^{+}, \mathrm{J}=0, \mathrm{M}=0\right)$. The EFD maps manifest a rich and surprising structure in this simplest of systems; for example, they show that the EFD is not necessarily synchronous with the nuclear flux density and can alternate in direction several times over the length of the molecule. The second application is to coherent tunnelling isomerization in the model inorganic system $\mathrm{B}_{4}$, in which all CENFs are synchronous. The contributions of core and valence electrons to the EFD are separately computed and it is found that core electrons flow with the nuclei, whereas the valence electrons flow obliquely to the core electrons in distinctive patterns. The third application is to the Cope rearrangement of semibullvalene, which also involves coherent tunnelling. An especially interesting discovery is that the so-called "pericyclic" electrons do not behave in the manner typically portrayed by the traditional Lewis structures with appended arrows. Indeed, it is found that only about 3 pericyclic electrons flow, in contrast to the 6 predicted by the Lewis picture. It is remarkable that the time scales of these three processes vary by 18 orders of magnitude: femtoseconds $\left(\mathrm{H}_{2}{ }^{+}\left({ }^{2} \Sigma_{\mathrm{g}}{ }^{+}\right)\right)$; picoseconds $\left(\mathrm{B}_{4}\right)$; kilosceconds (semibullvalene). It is emphasized that results presented herein are appearing in the literature for the first time.
\end{abstract}

Received 9th July 2015, Accepted 16th September 2015

DOI: $10.1039 / \mathrm{c} 5 \mathrm{cp} 03982 \mathrm{~g}$

www.rsc.org/pccp
The specific pathways of the particles constitute the mechanism of the process. For processes taking place in the electronic ground state, one typically employs the Born-Oppenheimer approximation (see Section 2) and monitors the time-development of the probability densities of the particles $\rho(\mathbf{q}, t)$ (i.e., the rate of change of the probability that the particles are observed in unit (hyper-) volume about the prescribed configuration q). Though $\rho(\mathbf{q}, t)$ tells one where the particles are at a given time, it does not indicate how they get there. More detailed information on the pathways can, in principle, be furnished by the flows of electrons and nuclei that attend the process. The most detailed quantitative description of the flow is provided by time sequences of threedimensional (vector) maps of the particle flux density, $\mathbf{j}(\mathbf{x}, t)$ (i.e., the instantaneous rate of flow of particles (electrons or nuclei) per unit area at points of observation $\mathbf{x}$ at time $t$ ). A less detailed 
description is given by the flux (i.e., the rate of flow of particles through a specified surface of observation).

We focus in this perspective on state-of-the-art theory and calculations of concerted flows of electrons and nuclei that accompany intramolecular processes (such as vibration, dissociation and isomerization) occurring in the electronic ground state (i.e., at such low energy that the coupling to electronic excited states is negligible) of the isolated molecule. On one hand, this field of research is relatively new, in that the first highly accurate quantum-mechanical calculations of electronic and nuclear fluxes were reported only in 2009 for the simplest molecular system, to which we refer subsequently as the "prototype": vibrating, aligned $\mathrm{H}_{2}{ }^{+}\left({ }^{2} \Sigma_{\mathrm{g}}{ }^{+}\right) .{ }^{1}$ On the other hand, the current effort has its roots in Schrödinger's seminal paper of 1926, in which he not only postulated his equation for the time-evolution of the state of a system but also defined the flux density. ${ }^{2}$ However, since the duration of most molecular processes is much shorter than that could be experimentally

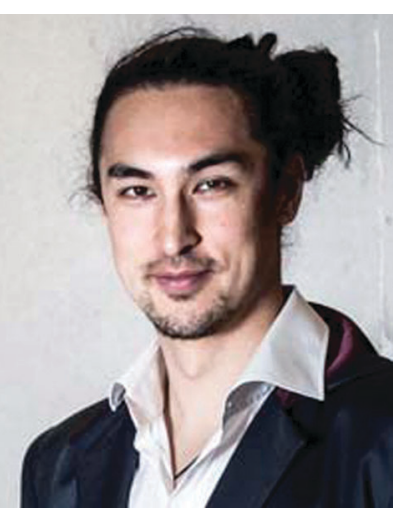

Timm Bredtmann
Timm Bredtmann was born in Essen, Germany in 1981. He studied chemistry at four universities in three different countries, receiving the MSc from the Freie Universität Berlin (FUB) in 2009. Under the guidance of Jörn Manz and Beate Paulus he earned the PhD ("Electron-Nuclear Quantum Fluxes in Adiabatic Chemical Processes") from the FUB in 2013. He is currently a research associate in the Max-BornInstitut Berlin, where he works with Mikhail Ivanov in the area of quantum dynamics in strong laser fields.

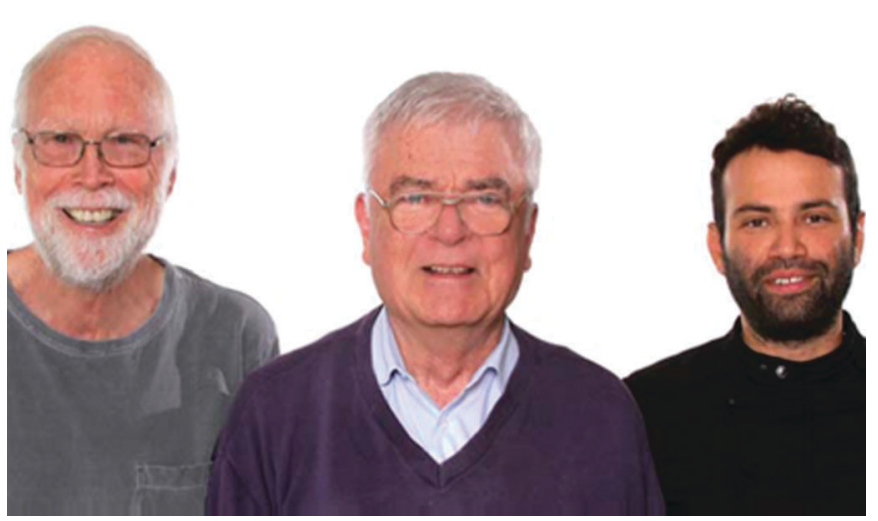

Dennis J. Diestler, Jörn Manz and Jhon Fredy Pérez-Torres resolved in Schrödinger's day, and indeed, for many decades thereafter, the field lay dormant until recently.

It is intuitively obvious that the electrons and nuclei must flow as the system rearranges from the reactant to the product configuration during a chemical reaction. These fluxes are generated spontaneously. However, detailed features of intramolecular fluxes remain unknown and many questions remain unanswered. Do the nuclei and electrons flow synchronously, or sequentially? As the original bonds of the reactant are broken and new bonds of the product form, do the electrons first flow out of the original bonds and then into the new ones, or are these fluxes synchronous? Do all nuclei and all electrons contribute to the fluxes, or only some of them? Do electrons and nuclei flow essentially in the same direction, or in oblique directions? What are the time scales of the fluxes? This perspective provides answers to all of these questions, with exemplary applications to model systems from physical (Section 3), inorganic (Section 4) and organic (Section 5) chemistry.

Fluxes are interesting and important because they provide information that complements that given by electronic and nuclear probability densities, which can be monitored by timeresolved spectroscopy or scattering. The complementarity of the information given by probability densities and fluxes, or flux densities, can be understood through a simple textbook example. Consider a free particle with mass $m$ described by the wave function $\psi(x, t)=N \exp (i k x-i E t / \hbar)$, where $k$ is the wave number and $E$ is the energy. The corresponding momentum and velocity are $p=\hbar k$ and $v=p / m$. The probability density that the particle is observed at point $x$ at time $t$ is $\rho(x, t)=|\psi(x, t)|^{2}=N^{2}$, whereas the flux (or flux density) observed at point $x$ at time $t$ is $j(x, t)=v \rho(x, t)=$ $v N^{2}$. The probability density is evidently the same for all particles, irrespective of their velocity, whereas the flux density depends on the velocity of the particle. Hence, the flux density discriminates among particles with different velocities, all of which have the same probability density. This simple example shows that a complete description of the evolving system requires knowledge of the flux densities, in addition to the probability densities.

Dennis Diestler was born in Ames, IA, USA in 1941. He received the $B S$ in chemistry from Harvey Mudd College in 1964, and the PhD from California Institute of Technology in 1968. He has held professorships at the University of Missouri (1967-1969), Purdue University (1969-1992), and the University of Nebraska (19922009), where he is presently emeritus professor. Jörn Manz was born in Hamburg, Germany in 1947. His autobiography appears in the Jörn Manz Festschrift of the J. Phys. Chem. A, 2012, 116, 11043. Since retiring in 2012, he has been guest professor in the research group of Professor Beate Paulus, his successor in the Freie Universität Berlin (FUB), and since 2013, has been senior guest professor in Shanxi University. Jhon Fredy Pérez-Torres was born in Medellin, Colombia in 1982. He received the BS in chemistry from Universidad de Antioquia in 2004, and the PhD in theoretical chemistry from the Universidad Autónoma de Madrid in 2012.

He was awarded the Colombian science prize Alejandro Angel Escobar in 2012. From 2012 to the present he has been a research associate in the FUB. DD was a teacher of JM and JM a teacher of JFP-T; all three are now colleagues and friends. 
The present, renewed interest in intramolecular fluxes, or flux densities, has been spurred indirectly by relatively recent experimental progress in the measurement of such dynamic properties as nuclear fluxes in chemical reactions in crossed molecular beams. ${ }^{3,4}$ Although these measurements provide valuable information on the mechanism of the reaction, expressed, for example, in terms of cross sections or state-to-state reaction probabilities, they tell one nothing about intramolecular fluxes generated during the reaction. Another indirect stimulus of the present interest in intramolecular fluxes is the measurement of circular electronic fluxes induced by weak magnetic fields in molecules such as benzene. ${ }^{5-7}$ However, even the strongest available magnetic fields (say 10 Tesla) can induce only extremely weak electronic fluxes, say of the order of one hundredth of an electron per period of one cycle. In contrast, the spontaneous fluxes that accompany intramolecular processes of interest here (see Sections 3-5) are one to two orders of magnitude greater, corresponding to transfers of one or more electrons during the process.

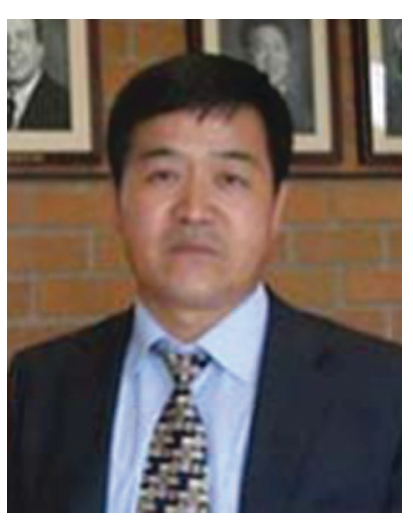

Si-Dian Li

Si-Dian Li was born in Yuncheng, China in 1959. He received the $B S$ from Beijing Normal University, the MS from Shanxi University, and the Doctor of Engineering from Xi'an Jiaotong University. $\mathrm{He}$ is presently professor of chemistry in the Institute of Molecular Science at Shanxi University. Since the 1990s he has collaborated in programs at Sussex University in the UK and Pacific Northwest National Laboratory in the US. He also serves as Vice President of Shanxi University. Professor Li's research interests focus on theoretical and experimental inorganic chemistry, computational chemistry, and materials sciences.
Experimental techniques for monitoring intramolecular fluxes directly are just emerging. Analysis of data from pumpprobe spectroscopy has yielded the first nuclear fluxes only recently. ${ }^{8}$ This fact serves to emphasize how timely a perspective on this topic is. Until recently it has received essentially no attention. Diverse systems remain to be investigated, with the possibility of new and surprising discoveries that may provide deeper insights into the mechanisms of fundamental molecular processes. For example, ref. 8 reports on four new quantum effects, one of which is dubbed the "quantum accordion effect" (which, in essence, refers to multiple alternations in direction of the quantum nuclear flux at a fixed time). This effect can also be observed in the concerted electronic and nuclear fluxes (CENFs) associated with vibration and dissociation in the prototype (see Section 3). Applications of the theory presented in Sections 3-5 reveal many additional, fascinating phenomena.

Theoretical studies related to those of primary concern in this perspective deal with, for example, purely nuclear fluxes during chemical reactions ${ }^{8-22}$ or purely electronic fluxes during

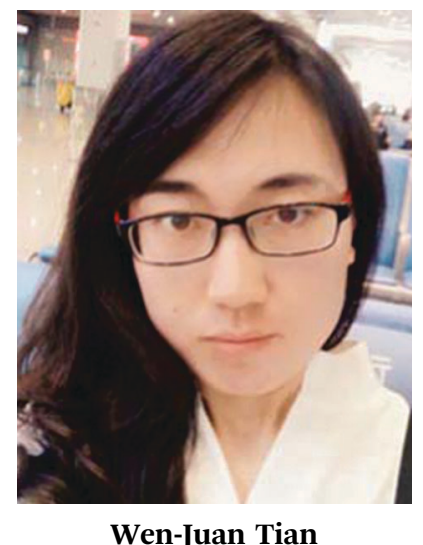

Wen-Juan Tian was born in Xinzhou, China in 1989. She received the $B S$ in chemistry from Taiyuan Normal University in 2011. She is currently pursuing the PhD under Professor Si-Dian $L i$ in Shanxi University. Her research focuses on the structures and bonding of boron and boron oxide clusters.

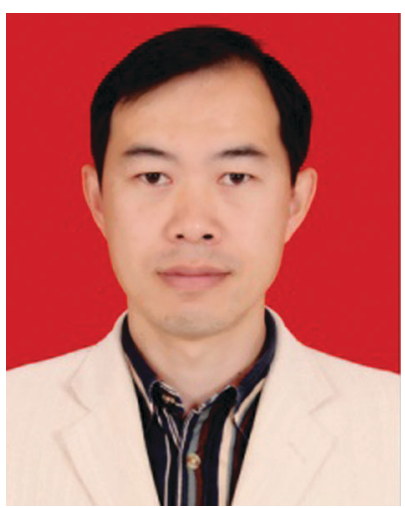

Yan-Bo Wu
Yan-Bo Wu was born in Jiamusi, China in 1978. He received the BS degree in chemical engineering from Dalian Polytechnic University, China in 2000. Upon receiving the $P h D$ in computational chemistry from Shanxi University in 2006, he served there as lecturer and was promoted to associate professor in 2009. He performed postdoctoral research at the University of Chinese Academy of Sciences in Beijing (2008-2010). Since 2013, he has been professor of chemistry at Shanxi University. Dr Wu's research interests are in the computational design and characterization of molecules with unusual bonding.

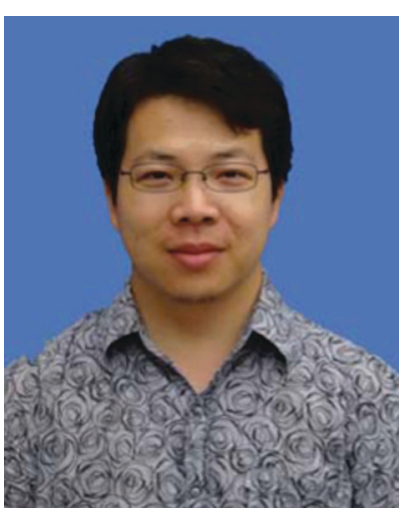

Yonggang Yang
Yonggang Yang was born in 1979, in Hubei, China. He received his MSc in physics in 2005, from the Chinese Academy of Sciences and his $P h D$ in theoretical chemistry from the Freie Universität Berlin in 2008, under adviser Oliver Kühn. Then he was a postdoctoral fellow with Markus Meuwly in the University of Basel. Since 2011, he has been an associate professor in Shanxi University, Taiyuan, China. His research interests include the theory of electronic and nuclear

fluxes during chemical reactions, hydrogen bonding and proton transfer, and vibrational spectra of small molecules via multidimensional quantum simulations. 
adiabatic $^{23-29}$ and diabatic ${ }^{30-38}$ processes (e.g., electronic ring currents in degenerate electronic excited states ${ }^{23-25}$ or electronic fluxes during diabatic reactions; ${ }^{30-38}$ see also ref. 39-41). The theoretical descriptions of purely electronic fluxes associated with these latter processes typically assume either that the nuclei are fixed ${ }^{23-25,30}$ or that classical mechanics can be used to describe nuclear motions. ${ }^{31-38}$ In contrast, ref. 1, which presents for the first time highly accurate simulations of CENFs for the prototype, handles the coupled electronic-nuclear motion completely by quantum mechanics. Subsequently, we have extended the quantum theory and its applications to more demanding systems, as well as to new phenomena of interest in physical, inorganic and organic chemistry, which focus on the relative magnitudes, directions, and synchronicities of CENFs. ${ }^{42-53}$ In addition we have studied interference effects in processes involving non-degenerate electronic states ${ }^{50,54}$ as well as transition current densities. ${ }^{55,56}$

This perspective is organized as follows. In Section 2 we develop the basic theory (see also Appendix A). Sections 3-5 are given to applications to the prototype, as well as to vibrating and dissociating $\mathrm{H}_{2}{ }^{+}\left({ }^{2} \Sigma_{\mathrm{g}}{ }^{+}, J=0, M=0\right.$ ) (which can be regarded as a "quantum bubble"), to isomerization of $\mathrm{B}_{4}$, and to the Cope rearrangement of semibullvalene (SBV) by coherent tunnelling. We note that as the complexity of these systems increases, the numbers of electrons and nuclei increase accordingly: $N_{\mathrm{e}}=1$, $N_{\mathrm{n}}=2$ for $\mathrm{H}_{2}{ }^{+} ; N_{\mathrm{e}}=20, N_{\mathrm{n}}=4$ for $\mathrm{B}_{4} ; N_{\mathrm{e}}=56, N_{\mathrm{n}}=16$ for SBV. It is also noteworthy that the durations of these processes range from femtoseconds $\left(\mathrm{H}_{2}^{+}\right)$over picoseconds $\left(\mathrm{B}_{4}\right)$ to kiloseconds (SBV). By the way, we emphasize that all of the results shown in this perspective are new, even though some of them are closely related to previously reported ones.

\section{Theory}

In this section we develop the fundamental quantum theory behind CENFs in a comprehensive, self-contained manner. The presentation

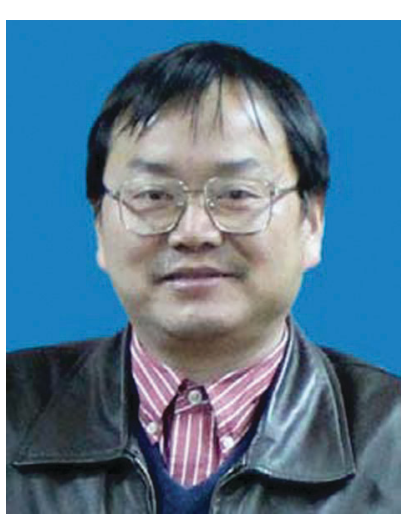

Hua-Jin Zhai
Hua-Jin Zhai was born in Ezhou, China in 1968. He studied physics at Wuhan University (BS, 1990) and the Chinese Academy of Sciences (PhD, 1998), and conducted postdoctoral research in physical chemistry at Washington State University and Pacific Northwest National Laboratory (WSU/PNNL). He was formerly a research scientist at WSU/PNNL and a senior research scientist at Brown University. Since 2013, Dr Zhai has been a distinguished professor in the Institute of Molecular Science, Shanxi University. His research interests are centered in experimental physical chemistry, materials chemistry, computational chemistry, and cluster physics. combines material that has appeared previously ${ }^{1,42-52}$ with important unpublished extensions. Sections 2.1-2.2 describe the system in detail and establish the notation and terminology. In Section 2.3 we introduce Hirschfelder's mobile coordinates, ${ }^{57-59}$ or analogous mobile-type coordinates, which are particularly advantageous because the corresponding nuclear kinetic energy is diagonal. Section 2.4 discusses the reduction of the number of coordinates in order to define one-dimensional models involving only a single one of the mobile-type coordinates. In Sections 2.5 and 2.6 we define population densities and flux densities. We also describe how the continuity equations that relate them can be reduced from three dimensions to one. Section 2.7 relates the flux densities in the nuclear center of mass (NCM) and internal-coordinate frames. Section 2.8 focuses on an important special scenario, namely CENFs during coherent tunnelling. We emphasize that all of the results of Sections 2.1-2.8 are general, depending on no special approximations, in particular the Born-Oppenheimer approximation (BOA). Finally, in Section 2.9 we summarize the BOA, pointing out its virtues for standard applications, but emphasizing a major fault that is critical for our present purpose: the BOA always yields a vanishing electronic flux density. We close Section 2 with a presentation of two fixes of this defect: (1) use of the reduced electronic continuity equation (as described in Section 2.6); (2) the coupled-channels technique. ${ }^{46-49,51}$

\subsection{Description of the system and Hamiltonians}

In the laboratory coordinate representation the complete Hamiltonian of a system comprising $N_{\mathrm{e}}$ electrons and $N_{\mathrm{n}}$ nuclei is expressed in the non-relativistic approximation as

$$
H_{\mathrm{T}}=-\frac{\hbar^{2}}{2 m_{\mathrm{e}}} \sum_{i=1}^{N_{\mathrm{e}}} \nabla_{\mathbf{r}_{i}}{ }^{2}-\sum_{a=1}^{N_{\mathrm{n}}} \frac{\hbar^{2}}{2 M_{a}} \nabla_{\mathbf{R}_{a}}{ }^{2}+V(\mathbf{r}, \mathbf{R})
$$

where $\mathbf{r}$ and $\mathbf{R}$ denote collections of electronic and nuclear Cartesian coordinates and $m_{\mathrm{e}}$ and $M_{a}$ stand for the electronic and nuclear masses. The Coulomb potential energy can be written in detail as

$$
V=V_{\mathrm{ee}}+V_{\mathrm{en}}+V_{\mathrm{nn}}
$$

where

$$
\begin{aligned}
V_{\mathrm{ee}} & =\frac{e^{2}}{8 \pi \varepsilon_{0}} \sum_{i=1}^{N_{\mathrm{e}}} \sum_{j \neq i}^{N_{\mathrm{e}}} \frac{1}{\left|\mathbf{r}_{i}-\mathbf{r}_{j}\right|} \\
V_{\mathrm{en}} & =-\frac{e^{2}}{4 \pi \varepsilon_{0}} \sum_{i=1}^{N_{\mathrm{e}}} \sum_{a=1}^{N_{\mathrm{n}}} \frac{Z_{a}}{\left|\mathbf{r}_{i}-\mathbf{R}_{a}\right|} \\
V_{\mathrm{nn}} & =\frac{e^{2}}{8 \pi \varepsilon_{0}} \sum_{a=1}^{N_{\mathrm{n}}} \sum_{b \neq a}^{N_{\mathrm{n}}} \frac{Z_{a} Z_{b}}{\left|\mathbf{R}_{a}-\mathbf{R}_{b}\right|}
\end{aligned}
$$

$e$ is the magnitude of the charge on the electron, $Z_{a}$ is the atomic number of nucleus $a$, and $\varepsilon_{0}$ is the permittivity of vacuum. The spins of particles are ignored, except insofar as they determine the permutation symmetry of the wave function. 
We transform the Hamiltonian to the total center-of-mass (COM) coordinate system, defined according to

$$
\begin{gathered}
\mathbf{S}_{\mathrm{T}}=M^{-1}\left[m_{\mathrm{e}} \sum_{i=1}^{N_{\mathrm{e}}} \mathbf{r}_{i}+\sum_{a=1}^{N_{\mathrm{n}}} M_{a} \mathbf{R}_{a}\right] \\
\mathbf{q}_{i}=\mathbf{r}_{i}-\mathbf{S} \\
Q_{\alpha}=\sum_{a=1}^{N_{\mathrm{n}}} \sum_{\gamma=x, y, z} W_{\alpha ; a \gamma} R_{a \gamma}, \quad \alpha=1,2, \ldots, 3 N_{\mathrm{n}}-3
\end{gathered}
$$

where

$$
\mathbf{S}=M_{\mathrm{n}}{ }^{-1}\left[\sum_{a=1}^{N_{\mathrm{n}}} M_{a} \mathbf{R}_{a}\right]
$$

and

$$
\begin{gathered}
M=N_{\mathrm{e}} m_{\mathrm{e}}+M_{\mathrm{n}} \\
M_{\mathrm{n}}=\sum_{a=1}^{N_{\mathrm{n}}} M_{a}
\end{gathered}
$$

Here $\mathbf{S}_{\mathrm{T}}$ stands for the COM of the entire system and $\mathbf{S}$ for the NCM. The coordinates of the electrons with respect to the NCM are given by eqn (4b) and the internal nuclear coordinates $Q_{\alpha}$ by eqn (4c), where $W_{\alpha ; a \gamma}$ are real constants. The $R_{a \gamma}$ stand for Cartesian components of the laboratory coordinates of the nuclei: $R_{1 x}=X_{1} ; R_{1 y}=Y_{1} ; R_{1 z}=Z_{1} ; R_{2 x}=X_{2} ; \ldots R_{N_{\mathrm{n}} z}=Z_{N_{\mathrm{n}}}$. The summation on $\gamma$ in eqn (4c) runs over the Cartesian components. We note that the internal coordinates are not unique and may be chosen for convenience. For example, in the case of the diatomic molecule $\mathrm{AB}$, for which $N_{\mathrm{n}}=2$, one typically takes the internal nuclear coordinates to be: $Q_{1}=R_{a x}-R_{b x}=X_{a}-X_{b}$; $Q_{2}=R_{a y}-R_{b y}=Y_{a}-Y_{b} ; Q_{3}=R_{a z}-R_{b z}=Z_{a}-Z_{b}$. We also designate the vector distance from nucleus $b$ to nucleus $a$ by $\mathbf{R}=Q_{1} \mathbf{e}_{x}+Q_{2} \mathbf{e}_{y}+Q_{3} \mathbf{e}_{z}$, where $\mathbf{e}$ denotes the Cartesian unit vector.

A tedious calculation yields

$$
\begin{aligned}
H_{\mathrm{T}}= & -\frac{\hbar^{2}}{2 M} \nabla_{\mathbf{S}_{\mathrm{T}}}{ }^{2}-\frac{\hbar^{2}}{2 \mu_{\mathrm{e}}} \sum_{i=1}^{N_{\mathrm{e}}} \nabla_{\mathbf{q}_{i}}{ }^{2}-\frac{\hbar^{2}}{2 M_{\mathrm{n}}} \sum_{i=1}^{N_{\mathrm{e}}} \sum_{j \neq i}^{N_{\mathrm{e}}} \nabla_{\mathbf{q}_{i}} \cdot \nabla_{\mathbf{q}_{j}} \\
& -\sum_{\alpha=1}^{3 N_{\mathrm{n}}-3} \sum_{\beta=1}^{3 N_{\mathrm{n}}-3} \frac{\hbar^{2}}{2 \mu_{\alpha \beta}} \frac{\partial^{2}}{\partial Q_{\alpha} \partial Q_{\beta}}+V(\mathbf{q}, \mathbf{Q}), \\
= & -\frac{\hbar^{2}}{2 M} \nabla_{\mathbf{S}_{\mathrm{T}}}{ }^{2}+H
\end{aligned}
$$

where $H$ is the “internal” Hamiltonian, the reduced masses are given by

$$
\begin{gathered}
\mu_{\mathrm{e}}=m_{\mathrm{e}} M_{\mathrm{n}} /\left(m_{\mathrm{e}}+M_{\mathrm{n}}\right) \\
\mu_{\alpha \beta}{ }^{-1}=\sum_{a=1}^{N_{\mathrm{n}}} \sum_{\gamma} \frac{W_{\alpha ; a \gamma} W_{\beta ; a \gamma}}{M_{a}}
\end{gathered}
$$

and $\mathbf{q}$ and $\mathbf{Q}$ stand for collections of coordinates.

\subsection{Wave functions}

Since the total COM contribution to $H_{\mathrm{T}}$ separates from the remainder (see eqn (7)), the entire wavefunction is factorable as

$$
\Psi_{\mathrm{T}}\left(\mathbf{S}_{\mathrm{T}}, \mathbf{q}, \mathbf{Q}, t\right)=\xi\left(\mathbf{S}_{\mathrm{T}}, t\right) \Psi(\mathbf{q}, \mathbf{Q}, t)
$$

The "external" COM factor $\xi$ obeys the Schrödinger equation

$$
i \hbar \frac{\partial \xi\left(\mathbf{S}_{\mathrm{T}}, t\right)}{\partial t}=-\frac{\hbar^{2}}{2 M} \nabla_{\mathbf{S}_{\mathrm{T}}}{ }^{2} \xi\left(\mathbf{S}_{\mathrm{T}}, t\right)
$$

and the "internal" factor $\Psi$ satisfies

$$
i \hbar \frac{\partial \Psi(\mathbf{q}, \mathbf{Q}, t)}{\partial t}=H \Psi(\mathbf{q}, \mathbf{Q}, t)
$$

The internal Hamiltonian can be written

$$
H=H_{\mathrm{e}}+T_{\mathrm{n}}
$$

where

$$
\begin{gathered}
H_{\mathrm{e}}=-\frac{\hbar^{2}}{2 \mu_{\mathrm{e}}} \sum_{i=1}^{N_{\mathrm{e}}} \nabla_{\mathbf{q}_{i}}{ }^{2}-\frac{\hbar^{2}}{2 M_{\mathrm{n}}} \sum_{i=1}^{N_{\mathrm{e}}} \sum_{j \neq i}^{N_{\mathrm{e}}} \nabla_{\mathbf{q}_{i}} \cdot \nabla_{\mathbf{q}_{j}}+V(\mathbf{q}, \mathbf{Q}) \\
T_{\mathrm{n}}=-\sum_{\alpha=1}^{3 N_{\mathrm{n}}-3} \sum_{\beta=1}^{3 N_{\mathrm{n}}-3} \frac{\hbar^{2}}{2 \mu_{\alpha \beta}} \frac{\partial^{2}}{\partial Q_{\alpha} \partial Q_{\beta}}
\end{gathered}
$$

The eigenfunctions of the internal Hamiltonian satisfy the eigenvalue equation

$$
H \psi_{n}(\mathbf{q}, \mathbf{Q})=E_{n} \psi_{n}(\mathbf{q}, \mathbf{Q})
$$

where $n$, which generally stands for a set of quantum numbers, specifies the eigenstate. The eigenfunctions comprise a complete, orthonormal set, i.e.,

$$
\int \mathrm{d} \mathbf{q} \int \mathrm{d} \mathbf{Q} \psi_{n}(\mathbf{q}, \mathbf{Q}) \psi_{m}(\mathbf{q}, \mathbf{Q})=\delta_{n m}
$$

(We remark that the spectrum includes continua and therefore the Kronecker delta is replaced by the Dirac delta where necessary.) The eigenfunctions can be taken to be real, but we note that in case of degenerate excited states they can as well be taken to be complex. Any well behaved function $f(\mathbf{q}, \mathbf{Q})$ in the internal $3\left(N_{\mathrm{e}}+N_{\mathrm{n}}-1\right)$-dimensional coordinate space can be expressed as a linear combination of the $\left\{\psi_{n}\right\}$. Hence, if the system is prepared in the state $\Psi(\mathbf{q}, \mathbf{Q}, 0)$ at the initial time $(t=0)$, the wave function at time $t$ can be represented in the basis of eigenfunctions of $H$ by

$$
\Psi(\mathbf{q}, \mathbf{Q}, t)=\sum_{n} c_{n} \exp \left(-i E_{n} t / \hbar\right) \psi_{n}(\mathbf{q}, \mathbf{Q})
$$

where the constant coefficients are

$$
c_{n}=\int \mathrm{d} \mathbf{q} \int \mathrm{d} \mathbf{Q} \psi_{n}(\mathbf{q}, \mathbf{Q}) \Psi(\mathbf{q}, \mathbf{Q}, 0)
$$

That $\Psi$ given by eqn (15) solves the internal Schrödinger equation can be seen by its direct substitution into eqn (10b). We emphasize that, in general, $\Psi$ is complex.

The electronically adiabatic processes with which we are concerned proceed without electronic excitations. In practice, this means that the summation in eqn (15) includes only eigenstates with sufficiently low energies $E_{n}$. For example, in the case of vibrating aligned $\mathrm{H}_{2}^{+}$(see Section 3.1), the summation is restricted to eigenstates having energies below the dissociation threshold. For dissociating aligned $\mathrm{H}_{2}{ }^{+}$, the summation includes 
only eigenfunctions that do not change sign as a function of $\mathbf{q}$ for fixed $\mathbf{Q}$, although they do so as a function of $\mathbf{Q}$ for fixed $\mathbf{q}$. In the cases of coherent tunnelling in $\mathrm{B}_{4}$ and SBV (see Sections 4 and 5) the summations are restricted to just the two states that comprise the lowest tunnelling doublet.

\subsection{Diagonalization of the kinetic energy}

We employ internal nuclear coordinates $Q_{\alpha}$ in terms of which the expression for the nuclear kinetic energy is diagonal (i.e., all cross terms $\alpha \neq \beta$ are absent from the expressions in eqn (7), (8b) and (12b)). Accordingly, we can rewrite eqn (12b) as

$$
T_{\mathrm{n}}=-\sum_{\alpha=1}^{3 N_{\mathrm{n}}-3} \frac{\hbar^{2}}{2 \mu_{\alpha}} \frac{\partial^{2}}{\partial Q_{\alpha}^{2}}
$$

where $\mu_{\alpha}{ }^{-1} \equiv \mu_{\alpha \alpha}{ }^{-1}=\sum_{a=1}^{N_{\mathrm{n}}} \sum_{\gamma} W_{\alpha ; a \gamma}{ }^{2} / M_{a}$. A useful example of such coordinates are the so-called "mobile" coordinates introduced by Hirschfelder, ${ }^{57-59}$ which include the three NCM coordinates as the subset $\left\{S_{\gamma}, \gamma=x, y, z\right\}$ as well as the $3 N_{\mathrm{n}}-3$ internal coordinates $Q_{\alpha}$. Alternative examples are found in the application of "mobiletype" nuclear coordinates in Sections 4 and 5. To simplify the terminology, we henceforward refer to all sets of nuclear coordinates that diagonalize the nuclear kinetic energy as "mobile-type". They can be expressed compactly by the matrix equation

$$
\tilde{\mathbf{Q}}=\tilde{\mathbf{W}} \mathbf{R}
$$

where the $3 N_{\mathrm{n}}$-dimensional column matrix $\mathbf{\mathbf { Q }}$ comprises the $Q_{\alpha}$ coordinates plus the NCM coordinates, the $3 N_{\mathrm{n}} \times 3 N_{\mathrm{n}}$ matrix $\tilde{\mathbf{W}}$ consists of the matrix $\mathbf{W}$ supplemented by an additional three rows at the bottom, and the $3 N_{\mathrm{n}}$-dimensional column matrix $\mathbf{R}$ consists of the Cartesian coordinates of the nuclei. Specifically, the elements of $\tilde{\mathbf{Q}}, \tilde{\mathbf{W}}$ and $\mathbf{R}$ are: $\tilde{Q}_{\alpha}=Q_{\alpha}$ for $1 \leq \alpha \leq 3 N_{\mathrm{n}}-3$; $\tilde{Q}_{3 N_{\mathrm{n}}-2}=S_{x}, \tilde{Q}_{3 N_{\mathrm{n}}-1}=S_{y}$ and $\tilde{Q}_{3 N_{\mathrm{n}}}=S_{z} ; \tilde{W}_{\alpha ; a \gamma}=W_{\alpha ; a \gamma}$ for $1 \leq \alpha \leq$ $3 N_{\mathrm{n}}-3 ; \quad \tilde{W}_{3 N_{\mathrm{n}}-2 ; a \gamma}=\left(M_{a} / M_{\mathrm{n}}\right) \delta_{\gamma x} ; \quad \tilde{W}_{3 N_{\mathrm{n}}-1 ; a \gamma}=\left(M_{a} / M_{\mathrm{n}}\right) \delta_{\gamma y} ;$ $\tilde{W}_{3 N_{\mathrm{n}} ; a \gamma}=\left(M_{a} / M_{\mathrm{n}}\right) \delta_{\gamma z} ;(\mathbf{R})_{1}=R_{1 x}=X_{1},(\mathbf{R})_{2}=R_{1 y}=Y_{1},(\mathbf{R})_{3}=$ $R_{1 z}=Z_{1},(\mathbf{R})_{4}=R_{2 x}=X_{2}, \ldots,(\mathbf{R})_{3 N_{\mathrm{n}}}=R_{N_{\mathrm{n}} z}=Z_{N_{\mathrm{n}}}$. The transformation (eqn (18)) is generally defined so that det $\tilde{\mathbf{W}}=1$.

By the chain rule for differentiation we have

$$
\partial / \partial R_{a \gamma}=\sum_{\alpha=1}^{3 N_{\mathrm{n}}} \tilde{W}_{\alpha ; \alpha \gamma} \partial / \partial \tilde{Q}_{\alpha}
$$

Multiplying eqn (19) by $-i \hbar$, we recognize that the momenta conjugate to the Cartesian laboratory coordinates $\left(P_{a \gamma} \equiv-i \hbar \partial / \partial R_{a \gamma}\right)$ are related to their mobile-type counterparts $\left(\tilde{P}_{\alpha} \equiv-i \hbar \partial / \partial \tilde{Q}_{\alpha}\right)$ by the matrix equation

$$
\mathbf{P}=\tilde{\mathbf{W}}^{\mathrm{T}} \tilde{\mathbf{P}}
$$

where the superscript $\mathrm{T}$ denotes the transpose. Insertion of eqn (19) into the expression for the kinetic energy in laboratory coordinates yields the sum of the internal nuclear kinetic energy and the NCM kinetic energy

$$
\mathrm{KE} \equiv-\sum_{a=1}^{N_{\mathrm{n}}} \sum_{\gamma} \frac{\hbar^{2}}{2 M_{a}} \frac{\partial^{2}}{\partial R_{a \gamma^{2}}{ }^{2}}=-\sum_{\alpha=1}^{3 N_{\mathrm{n}}-3} \frac{\hbar^{2}}{2 \mu_{\alpha}} \frac{\partial^{2}}{\partial Q_{\alpha}^{2}}-\frac{\hbar^{2}}{2 M_{\mathrm{n}}} \sum_{\gamma} \frac{\partial^{2}}{\partial S_{\gamma}{ }^{2}}
$$

where $\mu_{\alpha}$ denotes the reduced mass associated with the $\alpha$ th mobile-type coordinate. In the instance of the diatomic $\mathrm{AB}$, $N_{\mathrm{n}}=2$, the (internal) mobile-type coordinates are $Q_{1}=X_{a}-X_{b}$, $Q_{2}=Y_{a}-Y_{b}$, and $Q_{3}=Z_{a}-Z_{b}$, and the reduced mass is $\mu_{1}=\mu_{2}=$ $\mu_{3}=\mu_{a b}=M_{a} M_{b} / M_{\mathrm{n}}$. The general expression in eqn (21) reduces to

$$
\mathrm{KE}=-\frac{\hbar^{2}}{2 \mu_{a b}} \sum_{\alpha=1}^{3} \frac{\partial^{2}}{\partial Q_{\alpha^{2}}}-\frac{\hbar^{2}}{2 M_{\mathrm{n}}} \sum_{\gamma} \frac{\partial^{2}}{\partial S_{\gamma}^{2}}
$$

where the first term refers to the (internal) kinetic energy of A relative to $\mathrm{B}$ and the second term to that of the NCM. For polyatomic molecules consisting of three or more nuclei the mobile-type (or Jacobi) coordinates may be constructed successively as distances between COMs of subgroups of nuclei. We consider the triatomic molecule $\mathrm{ABC}$, for example. We suppose that nuclei A and B constitute two subgroups and take the first set of mobile-type coordinates to be $Q_{1}=R_{a x}-R_{b x}, Q_{2}=R_{a y}-$ $R_{b y}, Q_{3}=R_{a z}-R_{b z}$ (the Cartesian components of the vector distance between A and B, i.e., the distance between the COMs of the subgroups). The associated reduced masses are $\mu_{1}=\mu_{2}=$ $\mu_{3}=M_{a} M_{b} /\left(M_{a}+M_{b}\right)$. To get the other mobile-type coordinates we form two subgroups: one comprising A and B and the other consisting of just C. The second set of mobile-type coordinates is then $Q_{4}=\left(M_{a} R_{a x}+M_{b} R_{b x}\right) /\left(M_{a}+M_{b}\right)-R_{c x}$, etc., and the reduced masses are $\mu_{4}=\mu_{5}=\mu_{6}=M_{c}\left(M_{a}+M_{b}\right) / M_{\mathrm{n}}$. This procedure is readily extended to larger polyatomic systems (see Sections 4, 5, and Appendix B). It can be shown that $\mu_{j}$ for the $j$ th mobile-type coordinate is just the reduced mass of the subgroups of nuclei connected by $j .^{57-59}$

Subsequently we employ the following more-or-less standard approximations:

(i) The COM is replaced by the NCM. As a consequence, $M$, $\mathbf{S}_{\mathrm{T}}$ and $\nabla_{\mathbf{S}_{\mathrm{T}}}$ in eqn (7), (9) and (10a) are replaced by $M_{\mathrm{n}}, \mathbf{S}$ and $\nabla_{\mathbf{s}}$, respectively;

(ii) the so-called mass-polarization contribution to the electronic kinetic energy (third term in eqn (7) and second term in eqn (12a)) is neglected;

(iii) the reduced mass of the electron $\mu_{\mathrm{e}}$ is replaced by the electron mass $m_{\mathrm{e}}$ in eqn (7), (8a) and (12a). Now with these approximations and a choice of nuclear coordinates that produces a diagonal kinetic energy, the original internal Hamiltonian (see eqn (12)) becomes

$$
H=H_{\mathrm{e}}+T_{\mathrm{n}}
$$

where

$$
\begin{gathered}
H_{\mathrm{e}}=-\frac{\hbar^{2}}{2 m_{\mathrm{e}}} \sum_{i=1}^{N_{\mathrm{e}}} \nabla_{\mathbf{q}_{i}}^{2}+V(\mathbf{q}, \mathbf{Q}) \\
T_{\mathrm{n}}=-\sum_{\alpha=1}^{3 N_{\mathrm{n}}-3} \frac{\hbar^{2}}{2 \mu_{\alpha}} \frac{\partial^{2}}{\partial Q_{\alpha^{2}}}
\end{gathered}
$$

For notational simplicity, we use the same symbols $H, H_{\mathrm{e}}$, and $T_{\mathrm{n}}$ as for the exact quantities. 


\subsection{One-dimensional models}

We occasionally employ models in which the number of nuclear degrees of freedom is reduced to a single special one. For example, in the case of the diatomic $\mathrm{AB}$, the normal threedimensional nuclear kinetic-energy operator is $T_{\mathrm{n}}=-\sum_{\alpha=1}^{3} \frac{\hbar^{2}}{2 \mu_{\alpha}} \frac{\partial^{2}}{\partial Q_{\alpha}^{2}}$. If we assume $\mathrm{AB}$ to be aligned or oriented parallel to the $z$-axis (as can be accomplished by methods for alignment ${ }^{60-65}$ or orientation $^{66-68}$ of molecules), for example, then nuclear motion in the $x$ - and $y$-directions is suppressed (i.e., we set $Q_{1}=X_{a}-X_{b}=0$ and $\left.Q_{2}=Y_{a}-Y_{b}=0\right)$. That is, the dimensionality of the nuclear motion is reduced from three (3D) to one (1D). As a consequence the kinetic-energy operator becomes $T_{\mathrm{n}}=-\frac{\hbar^{2}}{2 \mu_{3}} \frac{\partial^{2}}{\partial Q_{3}^{2}}$ (see Section 3.1).

In general, we define a 1D model by fixing all internal nuclear coordinates, save a special one, say $Q_{\alpha}$, and the $\operatorname{NCM}\left\{S_{x}=S_{y}=S_{z}=0\right\}$. The inversion of eqn (18) then gives

$$
R_{a \gamma}=\left[\tilde{\mathbf{W}}^{-1}\right]_{a \gamma ; \alpha} \tilde{Q}_{\alpha}
$$

which provides a map of the movements of the special internal nuclear coordinate onto those of the Cartesian components of the nuclei in the NCM frame. Eqn (24) becomes

$$
\begin{gathered}
H_{\mathrm{e}}=-\frac{\hbar^{2}}{2 m_{\mathrm{e}}} \sum_{i=1}^{N_{\mathrm{e}}} \nabla_{\mathbf{q}_{i}}{ }^{2}+V\left(\mathbf{q}, Q_{\alpha}\right) \\
T_{\mathrm{n}}=-\frac{\hbar^{2}}{2 \mu_{\alpha}} \frac{\partial^{2}}{\partial Q_{\alpha}{ }^{2}}
\end{gathered}
$$

and the wave function satisfies the Schrödinger equation

$$
i \hbar \partial \Psi\left(\mathbf{q}, Q_{\alpha}, t\right) / \partial t=\left(H_{\mathrm{e}}+T_{\mathrm{n}}\right) \Psi\left(\mathbf{q}, Q_{\alpha}, t\right)
$$

The applications of the $1 \mathrm{D}$ model to the prototype, $\mathrm{B}_{4}$, and semibullvalene in Sections 3-5 provide illustrations.

\subsection{Population densities and flux densities}

The generic classical expressions for the particle population density and particle population flux density are, respectively

$$
\begin{gathered}
\rho(\mathbf{x}, t) \equiv \sum_{i} \delta\left[\mathbf{x}-\mathbf{r}_{i}(t)\right] \\
\mathbf{j}(\mathbf{x}, t) \equiv \sum_{i} \delta\left[\mathbf{x}-\mathbf{r}_{i}(t)\right] \dot{\mathbf{r}}_{i}(t)
\end{gathered}
$$

where $\mathbf{r}_{i}$ and $\dot{\mathbf{r}}_{i}$ are the position and velocity of particle $i$, respectively, and $\mathbf{x}$ is the point of observation. The quantum formulas are given by expectation values of the corresponding operators. However, we normally wish to compute these quantities with respect to "internal" reference points, instead of the laboratory origin. In particular, the NCM serves as the most common reference. (We note that the role of translation of the NCM in the laboratory frame has been previously investigated. ${ }^{69,70}$ ) Thus, the electronic population density (EPD) is

$$
\begin{aligned}
\left\langle\rho_{\mathrm{e}, \mathrm{NCM}}(\mathbf{x}, t)\right\rangle & =\left\langle\Psi(t)\left|\sum_{i=1}^{N_{\mathrm{e}}} \delta\left(\mathbf{x}-\mathbf{q}_{i}\right)\right| \Psi(t)\right\rangle \\
& =\sum_{i=1}^{N_{\mathrm{e}}} \int \mathrm{d} \mathbf{Q} \prod_{j \neq i}^{N_{\mathrm{e}}} \int \mathrm{d} \mathbf{q}_{j}|\Psi(\mathbf{q}, \mathbf{Q}, t)|_{\mathbf{q}_{i}=\mathbf{x}}^{2} \\
& =N_{\mathrm{e}} \int \mathrm{d} \mathbf{Q} \prod_{j \neq 1}^{N_{\mathrm{e}}} \int \mathrm{d} \mathbf{q}_{j}|\Psi(\mathbf{q}, \mathbf{Q}, t)|_{\mathbf{q}_{1}=\mathbf{x}}^{2}
\end{aligned}
$$

where $\mathbf{q}_{i}$ is the distance of the $i$ th electron from the NCM and $\mathbf{x}$ is the distance of the point of observation from the NCM. The third line of eqn (29a) invokes the equivalence (indistinguishability) of the electrons. Likewise the electronic flux density (EFD) can be shown to be ${ }^{49}$

$$
\begin{aligned}
\left\langle\mathbf{j}_{\mathrm{e}, \mathrm{NCM}}(\mathbf{x}, t)\right\rangle & =\operatorname{Re}\left\{\left\langle\Psi(t)\left|\sum_{i=1}^{N_{\mathrm{e}}} \delta\left(\mathbf{x}-\mathbf{q}_{i}\right) \dot{\mathbf{q}}_{i}\right| \Psi(t)\right\rangle\right\} \\
& =N_{\mathrm{e}} \frac{\hbar}{2 i m_{\mathrm{e}}} \int \mathrm{d} \mathbf{Q} \prod_{j \neq 1}^{N_{\mathrm{e}}} \int \mathrm{d} \mathbf{q}_{j}\left[\Psi^{*} \nabla_{\mathbf{q}_{1}} \Psi-\Psi \nabla_{\mathbf{q}_{1}} \Psi^{*}\right]_{\mathbf{q}_{1}=\mathbf{x}}
\end{aligned}
$$

(see also Appendix A). Here the notation $\Pi \int d \mathbf{q}$ should be understood as $\int \prod \mathrm{d} \mathbf{q}$, and the product $\delta(x-q) \dot{q}$ should be interpreted as a symmetrized Hermitian term $[\delta(x-q) p+p \delta(x-q)] / 2 m_{\mathrm{e}}$.

The probability density and flux density for a particular nucleus $a$ in the NCM frame are given respectively by

$$
\begin{gathered}
\left\langle\rho_{a, \mathrm{NCM}}(\mathbf{x}, t)\right\rangle=\left\langle\Psi(t)\left|\delta\left[\mathbf{x}-\left(\mathbf{R}_{a}-\mathbf{S}\right)\right]\right| \Psi(t)\right\rangle \\
\left\langle\mathbf{j}_{a, \mathrm{NCM}}(\mathbf{x}, t)\right\rangle=\operatorname{Re}\left\{\left\langle\Psi(t)\left|\delta\left[\mathbf{x}-\left(\mathbf{R}_{a}-\mathbf{S}\right)\right]\left(\dot{\mathbf{R}}_{a}-\dot{\mathbf{S}}\right)\right| \Psi(t)\right\rangle\right\}
\end{gathered}
$$

\subsection{Continuity equations in three and one dimensions}

The EPD and EFD satisfy the three-dimensional (3D) continuity equation $(\mathrm{CE})^{71}$

$$
\partial\left\langle\rho_{\mathrm{e}, \mathrm{NCM}}(\mathbf{x}, t)\right\rangle / \partial t+\nabla_{\mathbf{x}} \cdot\left\langle\mathbf{j}_{\mathrm{e}, \mathrm{NCM}}(\mathbf{x}, t)\right\rangle=0
$$

which can be written explicitly in Cartesian coordinates as

$$
\partial\langle\rho(\mathbf{x}, t)\rangle / \partial t+\partial\left\langle j_{x}(\mathbf{x}, t)\right\rangle / \partial x+\partial\left\langle j_{y}(\mathbf{x}, t)\right\rangle / \partial y+\partial\left\langle j_{z}(\mathbf{x}, t)\right\rangle / \partial z=0
$$

where we temporarily drop the subscript "e,NCM" to lessen the notational burden. The $3 \mathrm{D} \mathrm{CE}$ is a direct consequence of the Schrödinger equation (eqn (10) or (A.2) of Appendix A). The $z$-component of the EFD, for example, is given by

$$
\left\langle j_{z}(\mathbf{x}, t)\right\rangle=N_{\mathrm{e}} \frac{\hbar}{2 i m_{\mathrm{e}}} \int \mathrm{d} \mathbf{Q} \prod_{j \neq 1}^{N_{\mathrm{e}}} \int \mathrm{d} \mathbf{q}_{j}\left[\Psi^{*} \frac{\partial}{\partial q_{1 z}} \Psi-\Psi \frac{\partial}{\partial q_{1 z}} \Psi^{*}\right]_{\mathbf{q}_{1}=\mathbf{x}}
$$

It is unfortunate that one cannot, in general, solve eqn (31b) to obtain formulas for the components of the EFD in terms of the EPD. Nevertheless, useful expressions for the electronic probability flux can be derived. For example, integrating eqn (31b) 
over the infinite plane normal to the $z$-axis yields

$$
\begin{aligned}
\int_{-\infty}^{\infty} \mathrm{d} x \int_{-\infty}^{\infty} \mathrm{d} y \partial\langle\rho(\mathbf{x}, t)\rangle / \partial t= & -\int_{-\infty}^{\infty} \mathrm{d} x \int_{-\infty}^{\infty} \mathrm{d} y \partial\left\langle j_{x}(\mathbf{x}, t)\right\rangle / \partial x \\
& -\int_{-\infty}^{\infty} \mathrm{d} x \int_{-\infty}^{\infty} \mathrm{d} y \partial\left\langle j_{y}(\mathbf{x}, t)\right\rangle / \partial y \\
& -\int_{-\infty}^{\infty} \mathrm{d} x \int_{-\infty}^{\infty} \mathrm{d} y \partial\left\langle j_{z}(\mathbf{x}, t)\right\rangle / \partial z
\end{aligned}
$$

The first two terms on the right side of eqn (33) vanish. Consider the first term, for which the integration on $x$ can be done in closed form to give

$$
\begin{aligned}
& \int_{-\infty}^{\infty} \mathrm{d} x \int_{-\infty}^{\infty} \mathrm{d} y \partial\left\langle j_{x}(\mathbf{x}, t)\right\rangle / \partial x \\
& \quad=\int_{-\infty}^{\infty} \mathrm{d} y\left[\left.\left\langle j_{x}(\mathbf{x}, t)\right\rangle\right|_{x=\infty}-\left.\left\langle j_{x}(\mathbf{x}, t)\right\rangle\right|_{x=-\infty}\right]=0
\end{aligned}
$$

The second line is a consequence of the localization of the EFD in space (or, equivalently, of the property that the wave function is square integrable). Hence, eqn (31b) reduces to the $1 \mathrm{D} \mathrm{CE}$

$$
\begin{aligned}
\frac{\partial}{\partial t}\left\langle\rho_{1 \mathrm{D}, z}(z, t)\right\rangle & \equiv \int_{-\infty}^{\infty} \mathrm{d} x \int_{-\infty}^{\infty} \mathrm{d} y \partial\langle\rho(\mathbf{x}, t)\rangle / \partial t \\
& =-\int_{-\infty}^{\infty} \mathrm{d} x \int_{-\infty}^{\infty} \mathrm{d} y \partial\left\langle j_{z}(\mathbf{x}, t)\right\rangle / \partial z \\
& \equiv-\frac{\partial}{\partial z}\left\langle j_{1 \mathrm{D}, z}(z, t)\right\rangle
\end{aligned}
$$

In eqn (35) $\left\langle\rho_{1 \mathrm{D}, z}(z, t)\right\rangle$ is the population (probability) density of electrons in the range $\mathrm{d} z$ about $z$, regardless of their position in the plane normal to the $z$-axis and $\left\langle j_{1 \mathrm{D}, z}(z, t)\right\rangle$ is the flux of electrons in the range $\mathrm{d} z$ about $z$. (The subscripts on $\left\langle\rho_{1 \mathrm{D}, z}(z, t)\right\rangle$ and $\left\langle j_{1 \mathrm{D}, z}(z, t)\right\rangle$ are intended to distinguish these $1 \mathrm{D}$ quantities from the $3 \mathrm{D}$ ones, $\langle\rho(\mathbf{x}, t)\rangle$ and $\left\langle j_{z}(\mathbf{x}, t)\right\rangle$, respectively.) Integration of eqn (35) gives

$$
\left\langle j_{\mathrm{e}, \mathrm{NCM} ; 1 \mathrm{D}, z}(z, t)\right\rangle=-\frac{\partial}{\partial t} \int_{-\infty}^{z} \mathrm{~d} z^{\prime}\left\langle\rho_{\mathrm{e}, \mathrm{NCM} ; 1 \mathrm{D}, z}\left(z^{\prime}, t\right)\right\rangle
$$

where we again invoke the localization of the EFD in space and append the subscript "e,NCM" to remind one that the formula pertains to the EFD in the NCM frame. Eqn (36) tells us that the flux of electrons parallel with the $z$-axis at the plane of observation $(z)$ is equal to the rate of loss of electrons from the region $-\infty<z^{\prime} \leq z$ (see ref. 1). Analogous expressions for nuclear fluxes associated with a $1 \mathrm{D}$ reaction coordinate have been derived and applied previously. ${ }^{10-12}$

In Section 3.2 we consider vibrating and dissociating $\mathrm{H}_{2}{ }^{+}$in the state ${ }^{2} \Sigma_{\mathrm{g}}{ }^{+}(J=M=0)$. To handle this system, we start from the electronic continuity equation in spherical coordinates ${ }^{72}$

$$
\begin{gathered}
\frac{\partial\left\langle\rho_{\mathrm{e}}(r, \vartheta, \varphi, t)\right\rangle}{\partial t}+\frac{1}{r^{2}} \frac{\partial}{\partial r}\left(r^{2}\left\langle j_{\mathrm{e}}(r, \vartheta, \varphi, t)\right\rangle\right) \\
+\frac{1}{r \sin \vartheta} \frac{\partial}{\partial \vartheta}\left(\sin \vartheta\left\langle j_{\mathrm{e} \vartheta}(r, \vartheta, \varphi, t)\right\rangle\right) \\
+\frac{1}{r \sin \vartheta} \frac{\partial}{\partial \varphi}\left(\left\langle j_{\mathrm{e} \varphi}(r, \vartheta, \varphi, t)\right\rangle\right)=0
\end{gathered}
$$

where we again momentarily drop the subscript "NCM" for economy of notation. For this special state, the EPD is isotropic (i.e., $\left.\rho_{\mathrm{e}}(r, \vartheta, \varphi, t)=\rho_{\mathrm{e}}(r, t)\right)$ and, as a consequence, so is the EFD. ${ }^{52}$ Therefore, the angular components of the EFD vanish and the continuity equation reduces to the radial $\mathrm{CE}$

$$
\frac{\partial\left\langle\rho_{\mathrm{e}}(r, t)\right\rangle}{\partial t}+\frac{1}{r^{2}} \frac{\partial}{\partial r}\left(r^{2}\left\langle j_{\mathrm{er}}(r, t)\right\rangle\right)=0
$$

Eqn (38) can be solved for the radial component of the EFD:

$$
\left\langle j_{\mathrm{e}, \mathrm{NCM} ; 1 \mathrm{D}, r}(r, t)\right\rangle=-\frac{\partial}{\partial t}\left[r^{-2} \int_{0}^{r} \mathrm{~d} r^{\prime} r^{\prime 2}\left\langle\rho_{\mathrm{e}, \mathrm{NCM}}\left(r^{\prime}, t\right)\right\rangle\right]
$$

The vanishing of $\left\langle j_{\mathrm{e}, \mathrm{NCM} ; 1 \mathrm{D}, r}(r, t)\right\rangle$ in the limit $r \rightarrow 0$ is implicit in this formula. Eqn (39) is the analogue of eqn (36). An analogous expression obtains for the radial component of the nuclear flux density in terms of the isotropic nuclear probability density (NPD):

$$
\left\langle j_{\mathrm{n}, \mathrm{NCM} ; 1 \mathrm{D}, r}(R, t)\right\rangle=-\frac{\partial}{\partial t}\left[R^{-2} \int_{0}^{R} \mathrm{~d} R^{\prime} R^{\prime 2}\left\langle\rho_{\mathrm{n}, \mathrm{NCM}}\left(R^{\prime}, t\right)\right\rangle\right]
$$

For the applications of the theory to isomerization of $\mathrm{B}_{4}$ and SBV in Sections 4 and 5 we employ cylindrical coordinates $(r, \varphi, z)$, where $r$ now signifies the distance from the $z$-axis. The electronic continuity equation is then ${ }^{72}$

$$
\begin{aligned}
& \frac{\partial\left\langle\rho_{\mathrm{e}}(r, z, \varphi, t)\right\rangle}{\partial t}+\frac{1}{r} \frac{\partial\left(r\left\langle j_{\mathrm{e} r}(r, z, \varphi, t)\right\rangle\right)}{\partial r}+\frac{1}{r} \frac{\partial\left\langle j_{\mathrm{e} \varphi}(r, z, \varphi, t)\right\rangle}{\partial \varphi} \\
& +\frac{\partial\left\langle j_{\mathrm{e} z}(r, z, \varphi, t)\right\rangle}{\partial z}=0
\end{aligned}
$$

Operating upon eqn (41) with $\int_{-\infty}^{\infty} \mathrm{d} z \int_{0}^{\infty} \mathrm{d} r r \ldots$ yields

$$
\begin{aligned}
\frac{\partial}{\partial \varphi}\left\langle j_{\mathrm{e}, \mathrm{NCM} ; 1 \mathrm{D}, \varphi}(\varphi, t)\right\rangle & \equiv \frac{\partial}{\partial \varphi} \int_{-\infty}^{\infty} \mathrm{d} z \int_{0}^{\infty} \mathrm{d} r\left\langle j_{\mathrm{e} \varphi}(r, \varphi, z, t)\right\rangle \\
& =-\frac{\partial}{\partial t} \int_{-\infty}^{\infty} \mathrm{d} z \int_{0}^{\infty} \mathrm{d} r r\left\langle\rho_{\mathrm{e}}(r, \varphi, z, t)\right\rangle \\
& \equiv-\frac{\partial}{\partial t}\left\langle\rho_{\mathrm{e}, \mathrm{NCM} ; 1 \mathrm{D}, \varphi}(\varphi, t)\right\rangle
\end{aligned}
$$

which is analogous to eqn (38). Integrating eqn (42) with respect to $\varphi$ gives the $1 \mathrm{D}$ angular $\mathrm{CE}$

$$
\begin{gathered}
\left\langle j_{\mathrm{e}, \mathrm{NCM} ; 1 \mathrm{D}, \varphi}(\varphi, t)\right\rangle-\left\langle j_{\mathrm{e}, \mathrm{NCM} ; 1 \mathrm{D}, \varphi}\left(\varphi_{0}, t\right)\right\rangle \\
=-\frac{\partial}{\partial t} \int_{\varphi_{0}}^{\varphi} \mathrm{d} \varphi^{\prime}\left\langle\rho_{\mathrm{e}, \mathrm{NCM} ; 1 \mathrm{D}, \varphi}\left(\varphi^{\prime}, t\right)\right\rangle \\
=-\frac{\partial}{\partial t}\left\langle P_{\mathrm{e}, \mathrm{NCM} ; 1 \mathrm{D}}\left(\varphi, \varphi_{0}, t\right)\right\rangle
\end{gathered}
$$


where $\left\langle P_{\mathrm{e}, \mathrm{NCM} ; 1 \mathrm{D}}\left(\varphi, \varphi_{0}, t\right)\right\rangle$ signifies the probability of observing an electron (or, equivalently, the population of electrons) in the cylindrical sector bounded by half-planes at $\varphi$ and $\varphi_{0}$ (the planes are separated by $\Delta \varphi=\varphi-\varphi_{0}$ ). Eqn (43) shows that the $1 \mathrm{D}$ angular flux depends not only on the rate of change of the population in the sector but also on the angular flux through the reference plane at $\varphi_{0}$. In the applications to $\mathrm{B}_{4}$ and SBV in Sections 4 and 5 , we invoke molecular symmetry to find a reference plane on which $\left\langle j_{\mathrm{e}, \mathrm{NCM} ; 1 \mathrm{D}, \varphi}\left(\varphi_{0}, t\right)\right\rangle=0 .^{26,44}$

The 1D models described in Section 2.4 obey the following continuity equations for the electrons and for the special internal nuclear coordinate $Q_{\alpha}$ (see Appendix A):

$$
\begin{gathered}
\partial\left\langle\rho_{\mathrm{e}, \mathrm{NCM}}(\mathbf{x}, t)\right\rangle / \partial t+\nabla_{\mathbf{x}} \cdot\left\langle\mathbf{j}_{\mathrm{e}, \mathrm{NCM}}(\mathbf{x}, t)\right\rangle=0 \\
\partial\left\langle\rho_{\alpha}(Q, t)\right\rangle / \partial t+\partial\left\langle j_{\alpha}(Q, t)\right\rangle / \partial Q=0
\end{gathered}
$$

In eqn (44) $\left\langle\rho_{\mathrm{e}, \mathrm{NCM}}(\mathbf{x}, t)\right\rangle$ and $\left\langle\mathbf{j}_{\mathrm{e}, \mathrm{NCM}}(\mathbf{x}, t)\right\rangle$ are given by eqn (29), with $\mathbf{Q}$ replaced by $Q_{\alpha}$; the corresponding quantities for the special internal mode are

$$
\begin{gathered}
\left\langle\rho_{\alpha}(Q, t)\right\rangle=\int \mathrm{d} \mathbf{q}\left|\Psi\left(\mathbf{q}, Q_{\alpha}, t\right)\right|_{Q_{\alpha}=Q}^{2} \\
\left\langle j_{\alpha}(Q, t\rangle=\frac{\hbar}{2 i \mu_{\alpha}} \int \mathrm{d} \mathbf{q}\left[\Psi^{*}\left(\mathbf{q}, Q_{\alpha}, t\right) \frac{\partial}{\partial Q_{\alpha}} \Psi\left(\mathbf{q}, Q_{\alpha}, t\right)\right.\right. \\
\left.-\Psi\left(\mathbf{q}, Q_{\alpha}, t\right) \frac{\partial}{\partial Q_{\alpha}} \Psi^{*}\left(\mathbf{q}, Q_{\alpha}, t\right)\right]_{Q_{\alpha}=Q}
\end{gathered}
$$

From eqn (44b) we deduce the analogue of eqn (43):

$$
\begin{aligned}
\left\langle j_{\alpha}(Q, t)\right\rangle-\left\langle j_{\alpha}\left(Q_{0}, t\right)\right\rangle & =-\frac{\partial}{\partial t}\left\{\int_{Q_{0}}^{Q} \mathrm{~d} Q^{\prime}\left\langle\rho_{\alpha}\left(Q^{\prime}, t\right)\right\rangle\right\} \\
& =-\frac{\partial}{\partial t}\left\langle P_{\alpha}\left(Q, Q_{0}, t\right)\right\rangle
\end{aligned}
$$

We note that the parameter $Q$ means the particular value of the special coordinate $Q_{\alpha}$ at which the observation of the relevant quantity is made. In practice, we choose the reference $Q_{0}$ such that $\left\langle j_{\alpha}\left(Q_{0}, t\right)\right\rangle=0$.

In the applications of Sections 4 and 5 the total EPD $\left\langle\rho_{\mathrm{e}, \mathrm{NCM}}(\mathbf{x}, t)\right\rangle$ is decomposed into contributions from core and valence electrons. Since these are additive, the related fluxes of core and valence electrons are also additive. The individual contributions obey continuity equations analogous to eqn (44).

The units of the various probability densities and flux densities that have been thus far introduced are listed in Table 1. We note that in the limit that the generalized CE is reduced to one dimension, the resulting flux densities $(j)$ are equivalent to fluxes $(F)$ and both have dimensions of reciprocal time.

\subsection{Relation between nuclear flux densities in the NCM and internal coordinate frames}

We often desire to examine the flux densities associated with the Cartesian coordinates of individual nuclei in the NCM frame. Thus, we seek to express the latter in terms of the flux
Table $1 \mathrm{SI}$ and atomic units for probability densities, flux densities and fluxes

\begin{tabular}{lllll}
\hline Property & Eqn number & Dimension & $\mathrm{SI}$ & Atomic units \\
\hline$\rho$ & $(28 \mathrm{a})$ and (58) & $3 \mathrm{D}$ & $\mathrm{m}^{-3}$ & $\mathrm{a}_{0}^{-3}$ \\
$\mathbf{j}_{F}$ & $(28 \mathrm{~b})$ & $3 \mathrm{D}$ & $\mathrm{m}^{-2} \mathrm{~s}^{-1}$ & $\mathrm{a}_{0}{ }^{-2} \mathrm{E}_{\mathrm{h}} \hbar^{-1 a}$ \\
$F^{b}$ & - & $3 \mathrm{D}$ & $\mathrm{s}^{-1}$ & $\mathrm{E}_{\mathrm{h}} \hbar^{-1}$ \\
$\rho_{1 \mathrm{D}, z}$ & $(35)$ & $1 \mathrm{D}$ & $\mathrm{m}^{-1}$ & $\mathrm{a}_{0}{ }^{-1}$ \\
$j_{1 \mathrm{D}, z}$ & $(35)$ & $1 \mathrm{D}$ & $\mathrm{s}^{-1}$ & $\mathrm{E}_{\mathrm{h}} \hbar^{-1}$ \\
$F_{1 \mathrm{D}, z}$ & - & $1 \mathrm{D}$ & $\mathrm{s}^{-1}$ & $\mathrm{E}_{\mathrm{h}} \hbar^{-1}$ \\
$\rho_{1 \mathrm{D}, \varphi}$ & $(42)$ & $1 \mathrm{D}$ & $\mathrm{rad}^{-1}$ & $\mathrm{rad}^{-1}$ \\
$j_{1 \mathrm{D}, \varphi}$ & $(42)$ and (63) & $1 \mathrm{D}$ & $\mathrm{s}^{-1}$ & $\mathrm{E}_{\mathrm{h}} \hbar^{-1}$ \\
$F_{1 \mathrm{D}, \varphi}$ & - & $1 \mathrm{D}$ & $\mathrm{s}^{-1}$ & $\mathrm{E}_{\mathrm{h}} \hbar^{-1}$ \\
$\rho$ & $($ A.3) and (55) & $3 N \mathrm{D}$ & $\mathrm{m}^{-3 N}$ & $\mathrm{a}_{0}{ }^{-3 N}$ \\
$J_{i}$ & $($ A.7a) & $3 N \mathrm{D}$ & $\mathrm{m}^{-3 N+1} \mathrm{~s}^{-1}$ & $\mathrm{a}_{0}{ }^{-3 N+1} \mathrm{E}_{\mathrm{h}} \hbar^{-1}$ \\
$J_{\alpha}$ & $($ A.7b) & $3 N \mathrm{D}$ & $\mathrm{m}^{-3 N+1} \mathrm{~s}^{-1}$ & $\mathrm{a}_{0}{ }^{-3 N+1} \mathrm{E}_{\mathrm{h}} \hbar^{-1}$ \\
$F$ & - & $3 N \mathrm{D}$ & $\mathrm{s}^{-1}$ & $\mathrm{E}_{\mathrm{h}} \hbar^{-1}$
\end{tabular}

${ }^{a} \mathrm{E}_{\mathrm{h}}$ stands for twice the (absolute value of the) energy of electronic ground state of the hydrogen atom ("hartree"). ${ }^{b} F$ stands for the flux, which is generally expressed as a surface integral, $F=\int_{S} \mathrm{~d} S \mathbf{n} \cdot \mathbf{j}$. The dimension of the surface is one less than the dimension of space. ${ }^{c} N=3\left(N_{\mathrm{e}}+N_{\mathrm{n}}\right)$ or $N=3\left(N_{\mathrm{e}}+N_{\mathrm{n}}-1\right)$ with or without, respectively, the coordinates of the NCM.

densities associated with the special internal nuclear coordinate, which is more conveniently computed using eqn (46). For the sake of convenience we take the NCM to be at the origin of the laboratory frame (i.e., we set $S_{x}=S_{y}=S_{z}=0$ ).

We begin with the classical formula for flux density associated with the $\gamma$ Cartesian component of nucleus $a$

$$
j_{a \gamma}=\delta\left(R-R_{a \gamma}\right) \dot{R}_{a \gamma}
$$

where $R$, a parameter in the $1 \mathrm{D}$ space of the Cartesian component $R_{a \gamma}$, signifies the point at which the flux is observed, $R_{a \gamma}=$ $R$. The corresponding quantum flux density is

$$
\begin{aligned}
\left\langle j_{a \gamma}(R, t)\right\rangle & =\operatorname{Re}\left\{\left\langle\Psi(t)\left|\delta\left(R-R_{a \gamma}\right) \dot{R}_{a \gamma}\right| \Psi(t)\right\rangle\right\} \\
& =\frac{\hbar}{2 i M_{a}} \int \mathrm{d} \mathbf{q} \int \mathrm{d} Q_{\alpha} \delta\left(R-R_{a \gamma}\right)\left[\Psi^{*} \frac{\partial \Psi}{\partial R_{a \gamma}}-\Psi \frac{\partial \Psi^{*}}{\partial R_{a \gamma}}\right]
\end{aligned}
$$

where we consider the 1D model with the special coordinate $Q_{\alpha}$. The second line of eqn (48) relies on the relation $M_{a} \dot{R}_{a \gamma}=-i \hbar \partial / \partial R_{a \gamma}$. According to eqn (18) and (19), we have

$$
\begin{gathered}
R_{a \gamma}=\left[\tilde{\mathbf{W}}^{-1}\right]_{a \gamma ; \alpha} Q_{\alpha} \\
\frac{\partial}{\partial R_{a \gamma}}=\left[\tilde{\mathbf{W}}^{\mathrm{T}}\right]_{a \gamma ; \alpha} \frac{\partial}{\partial Q_{\alpha}}
\end{gathered}
$$

Substitution of these expressions into eqn (48) gives

$$
\begin{aligned}
\left\langle j_{a \gamma}(R, t)\right\rangle= & \frac{\hbar}{2 i M_{a}} \int \mathrm{d} \mathbf{q} \int \mathrm{d} Q_{\alpha} \delta\left[R-\left(\tilde{\mathbf{W}}^{-1}\right)_{a \gamma ; \alpha} Q_{\alpha}\right] \\
& \times\left\{\Psi^{*}\left(\mathbf{q}, Q_{\alpha}, t\right)\left[\tilde{\mathbf{W}}^{\mathrm{T}}\right]_{a \gamma ; \alpha} \frac{\partial \Psi\left(\mathbf{q}, Q_{\alpha}, t\right)}{\partial Q_{\alpha}}\right. \\
& \left.-\Psi\left(\mathbf{q}, Q_{\alpha}, t\right)\left[\tilde{\mathbf{W}}^{\mathrm{T}}\right]_{a \gamma ; \alpha} \frac{\partial \Psi^{*}\left(\mathbf{q}, Q_{\alpha}, t\right)}{\partial Q_{\alpha}}\right\}
\end{aligned}
$$

Using the property of the Dirac distribution that $\delta(a x)=$ $|a|^{-1} \delta(x)$, we rewrite eqn (50) as 


$$
\begin{aligned}
\left\langle j_{a \gamma}(R, t)\right\rangle= & \frac{\hbar}{2 i M_{a}} \int \mathrm{d} \mathbf{q} \int \mathrm{d} Q_{\alpha} \delta\left[-\left(\tilde{\mathbf{W}}^{-1}\right)_{a \gamma ; \alpha}\left[Q_{\alpha}-R /\left(\tilde{\mathbf{W}}^{-1}\right)_{a \gamma ; \alpha}\right]\right. \\
& \times\left\{\Psi^{*}\left[\tilde{\mathbf{W}}^{\mathrm{T}}\right]_{a \gamma ; \alpha} \frac{\partial \Psi}{\partial Q_{\alpha}}-\Psi\left[\tilde{\mathbf{W}}^{\mathrm{T}}\right]_{a \gamma ; \alpha} \frac{\partial \Psi^{*}}{\partial Q_{\alpha}}\right\} \\
= & \frac{\hbar}{2 i M_{a}\left|\left[\tilde{\mathbf{W}}^{-1}\right]_{a \gamma ; \alpha}\right|} \int \mathrm{d} \mathbf{q}\left[\Psi^{*} \frac{\partial \Psi}{\partial Q_{\alpha}}-\Psi \frac{\partial \Psi^{*}}{\partial Q_{\alpha}}\right]_{Q_{\alpha}=R /\left[\tilde{\mathbf{W}}^{-1}\right]_{a \gamma ; \alpha}} \\
= & \frac{\mu_{\alpha}}{M_{a}} \frac{\left[\tilde{\mathbf{W}}^{\mathrm{T}}\right]_{a \gamma ; \alpha}\left|\left[\tilde{\mathbf{W}}^{-1}\right]_{a \gamma ; \alpha}\right|}{}\left\langle j_{\alpha}\left(R /\left[\tilde{\mathbf{W}}^{-1}\right]_{a \gamma ; \alpha}, t\right)\right\rangle
\end{aligned}
$$

where the third line depends on eqn (45b). Setting $R=R_{a \gamma}$ and using eqn (49a), we get

$$
\left\langle j_{a \gamma}\left(R_{a \gamma}, t\right)\right\rangle=\frac{\mu_{\alpha}}{M_{a}} \frac{\left[\tilde{\mathbf{W}}^{\mathrm{T}}\right]_{a \gamma ; \alpha}}{\left|\left[\tilde{\mathbf{W}}^{-1}\right]_{a \gamma ; \alpha}\right|}\left\langle j_{\alpha}\left(Q_{\alpha}, t\right)\right\rangle
$$

Thus, the fluxes of the individual nuclei $a$ along Cartesian coordinates $R_{a \gamma}$ are proportional to the fluxes associated with the internal nuclear coordinate $\alpha$. In Section 4, we show how to determine the factors of proportionality without a knowledge of $\tilde{\mathbf{W}}^{-1}$ (see also Appendices C and D).

\subsection{Concerted electronic and nuclear fluxes during coherent tunnelling}

Coherent tunnelling can be generally described by a wave function that consists of a coherent superposition of just the two lowest eigenstates $\psi_{0}$ and $\psi_{1}$ of $H$ (see eqn (13)). These form the tunnelling doublet with a splitting of energy levels of

$$
\Delta E=E_{1}-E_{0}
$$

and a related tunneling time

$$
\tau=h / \Delta E
$$

and tunneling rate

$$
k=1 / \tau
$$

Following the treatment by Hund ${ }^{73}$ of coherent nuclear tunnelling during isomerization reactions, we define reactant $(\mathrm{R})$ and product (P) states by

$$
\begin{gathered}
\psi_{\mathrm{R}}(\mathbf{q}, \mathbf{Q})=N \psi_{0}(\mathbf{q}, \mathbf{Q})+N \psi_{1}(\mathbf{q}, \mathbf{Q}) \\
\psi_{\mathrm{P}}(\mathbf{q}, \mathbf{Q})=-N \psi_{0}(\mathbf{q}, \mathbf{Q})+N \psi_{1}(\mathbf{q}, \mathbf{Q})
\end{gathered}
$$

where the normalization constant is $N=1 / \sqrt{2}$. The wave functions at time $t$ corresponding to the initial $\mathrm{R}$ and $\mathrm{P}$ states are given, respectively, by

$$
\psi_{\mathrm{R}}(\mathbf{q}, \mathbf{Q}, t)=N \psi_{0}(\mathbf{q}, \mathbf{Q}) \exp \left(-i E_{0} t / \hbar\right)+N \psi_{1}(\mathbf{q}, \mathbf{Q}) \exp \left(-i E_{1} t / \hbar\right)
$$

$\psi_{\mathrm{P}}(\mathbf{q}, \mathbf{Q}, t)=-N \psi_{0}(\mathbf{q}, \mathbf{Q}) \exp \left(-i E_{0} t / \hbar\right)+N \psi_{1}(\mathbf{q}, \mathbf{Q}) \exp \left(-i E_{1} t / \hbar\right)$
The corresponding $3 N=3\left(N_{\mathrm{e}}+N_{\mathrm{n}}-1\right)$-dimensional probability densities are

$$
\rho_{\mathrm{R}}(\mathbf{q}, \mathbf{Q}, t)=\left|\psi_{\mathrm{R}}(\mathbf{q}, \mathbf{Q}, t)\right|^{2}=\rho_{\mathrm{R}}(\mathbf{q}, \mathbf{Q})+\Delta \rho(\mathbf{q}, \mathbf{Q}) \sin ^{2}(\pi t / \tau)
$$

$$
\rho_{\mathrm{P}}(\mathbf{q}, \mathbf{Q}, t)=\left|\psi_{\mathrm{P}}(\mathbf{q}, \mathbf{Q}, t)\right|^{2}=\rho_{\mathrm{P}}(\mathbf{q}, \mathbf{Q})-\Delta \rho(\mathbf{q}, \mathbf{Q}) \sin ^{2}(\pi t / \tau)
$$

where

$$
\begin{aligned}
& \rho_{\mathrm{R}}(\mathbf{q}, \mathbf{Q})=\left|\psi_{\mathrm{R}}(\mathbf{q}, \mathbf{Q})\right|^{2} \\
& \rho_{\mathrm{P}}(\mathbf{q}, \mathbf{Q})=\left|\psi_{\mathrm{P}}(\mathbf{q}, \mathbf{Q})\right|^{2}
\end{aligned}
$$

and

$$
\Delta \rho(\mathbf{q}, \mathbf{Q}) \equiv \rho_{\mathrm{P}}(\mathbf{q}, \mathbf{Q})-\rho_{\mathrm{R}}(\mathbf{q}, \mathbf{Q})
$$

is the difference between product and reactant probability densities. These expressions are entirely analogous to those derived for coherent nuclear tunnelling. ${ }^{18,19}$ The corresponding 3D EPD difference is given by

$$
\left\langle\Delta \rho_{\mathrm{e}, \mathrm{NCM}}(\mathbf{x})\right\rangle=\left.N_{\mathrm{e}} \int \mathrm{d} \mathbf{Q} \prod_{j \neq 1}^{N_{\mathrm{e}}} \int \mathrm{d} \mathbf{q}_{j} \Delta \rho(\mathbf{q}, \mathbf{Q})\right|_{\mathbf{q}_{1}=\mathbf{x}}
$$

and the $3\left(N_{\mathrm{n}}-1\right) \mathrm{D}$ NPD difference by

$$
\left\langle\Delta \rho_{\mathrm{n}}(\mathbf{Q})\right\rangle=\int \mathrm{d} \mathbf{q} \Delta \rho(\mathbf{q}, \mathbf{Q})
$$

Likewise the 3D EPDs evolve as

$$
\begin{aligned}
\left\langle\rho_{\mathrm{e}, \mathrm{NCM}}(\mathbf{x}, t)\right\rangle_{\mathrm{R}} & =\left\langle\rho_{\mathrm{e}, \mathrm{NCM}}(\mathbf{x})\right\rangle_{\mathrm{R}}+\left\langle\Delta \rho_{\mathrm{e}, \mathrm{NCM}}(\mathbf{x})\right\rangle \sin ^{2}(\pi t / \tau) \\
\left\langle\rho_{\mathrm{e}, \mathrm{NCM}}(\mathbf{x}, t)\right\rangle_{\mathrm{P}} & =\left\langle\rho_{\mathrm{e}, \mathrm{NCM}}(\mathbf{x})\right\rangle_{\mathrm{P}}-\left\langle\Delta \rho_{\mathrm{e}, \mathrm{NCM}}(\mathbf{x})\right\rangle \sin ^{2}(\pi t / \tau)
\end{aligned}
$$

and the NPDs as

$$
\begin{aligned}
& \left\langle\rho_{\mathrm{n}}(\mathbf{Q}, t)\right\rangle_{\mathrm{R}}=\left\langle\rho_{\mathrm{n}}(\mathbf{Q})\right\rangle_{\mathrm{R}}+\left\langle\Delta \rho_{\mathrm{n}}(\mathbf{Q})\right\rangle \sin ^{2}(\pi t / \tau) \\
& \left\langle\rho_{\mathrm{n}}(\mathbf{Q}, t)\right\rangle_{\mathrm{P}}=\left\langle\rho_{\mathrm{n}}(\mathbf{Q})\right\rangle_{\mathrm{P}}-\left\langle\Delta \rho_{\mathrm{n}}(\mathbf{Q})\right\rangle \sin ^{2}(\pi t / \tau)
\end{aligned}
$$

It is evident that the expressions for the total probability densities, as well as those for the electronic and nuclear densities, have the same form: a superposition of the densities of reactant $(\mathrm{R})$ or product $(\mathrm{P})$ and a sinusoidal time dependence. Thus, as time increases from $t=0$ to $t=\tau / 2$, half the tunneling time, P increases as $\sin ^{2}(\pi t / \tau)$ and $\mathrm{R}$ decreases as $\cos ^{2}(\pi t / \tau)$. At time $t=\tau / 2$ only product is present. Accordingly, negative and positive values of the difference $\Delta \rho(\mathbf{q}, \mathbf{Q}) \equiv \rho_{\mathrm{P}}(\mathbf{q}, \mathbf{Q})-\rho_{\mathrm{R}}(\mathbf{q}, \mathbf{Q})$ over the $3 N \mathrm{D}$ configuration space indicate the disappearance of reactants and the appearance of products during tunneling from $\mathrm{R}$ to $\mathrm{P}$. This observation suggests that a reasonable definition of the main direction of the flux is from the centers of the negative domains of $\Delta \rho(\mathbf{q}, \mathbf{Q})$ to the centers of positive ones. Analogous arguments hold for the main directions of the electronic and nuclear fluxes (i.e., they flow from negative to positive domains of $\left\langle\Delta \rho_{\mathrm{e}, \mathrm{NCM}}(\mathbf{x})\right\rangle$ and $\left\langle\Delta \rho_{\mathrm{n}}(\mathbf{Q})\right\rangle$, respectively). We anticipate that different patterns of the electronic $\left(\left\langle\Delta \rho_{\mathrm{e}, \mathrm{NCM}}(\mathbf{x})\right\rangle\right)$ and the nuclear $\left(\left\langle\Delta \rho_{\mathrm{n}}(\mathbf{Q})\right\rangle\right)$ flux densities imply 
different main directions of the fluxes of the electrons and the nuclei.

Concerning the main directions of the nuclear fluxes, we suggest an approximation based on the rather large nuclear masses (compared with the electron). The more massive the nuclei, the more localized are their probability densities. In the limit that the masses tend to infinity, the difference of the NPDs of $\mathrm{P}$ and $\mathrm{R}$ can be approximated by the expression $\delta\left(R-R_{a, \mathrm{P}}\right)-\delta\left(R-R_{a, \mathrm{R}}\right)$, where $R_{a, \mathrm{P}}$ and $R_{a, \mathrm{R}}$ are the classical positions of nucleus $a$ in the product and reactant. Hence the main directions of the fluxes of the individual nuclei (with the NCM fixed at the origin) are from their classical positions for $\mathrm{R}$ to those for $\mathrm{P}$. It is reasonable to define one of the nuclear mobile-type coordinates, say $Q_{1}$, to correspond to the Cartesian component of the main directions of the fluxes, with the corresponding first row of the transformation matrix $\tilde{\mathbf{W}}$ (see eqn (18)). The nuclear flux associated with $Q_{1}$ then lies approximately along the nuclear main direction. Hence, for models in reduced $1 \mathrm{D}$ dimensionality, the special coordinate $Q_{1}$ should satisfy this criterion. Examples are presented in Sections 4 and 5 on coherent tunnelling in $\mathrm{B}_{4}$ and SBV. In these examples, the main directions of the fluxes are illustrated by arrows. Symmetric molecules such as $\mathrm{B}_{4}$ have symmetry-adapted sets of arrows for the main directions. We remark that the small electron mass implies delocalized electronic probability densities that do not lend themselves to the sort of classical analysis of the main directions for the nuclei. Instead, one has to compute the $\left\langle\Delta \rho_{\mathrm{e}, \mathrm{NCM}}(\mathbf{x})\right\rangle$, from which the main directions of electronic fluxes are seen to be from the (symmetry-adapted) centers of the negative domains to the positive ones.

In the applications of Sections 4 and 5, the total EPD is a sum of core plus valence electron densities. The additivity of the individual contributions implies the additivity of corresponding fluxes of core and valence electrons, all of which satisfy expressions analogous to eqn (58).

In the remainder of this section, we focus on this type of $1 \mathrm{D}$ model, with the single nuclear coordinate $Q_{1}$ representing the coordinate for the main directions of the (symmetry-adapted set of) nuclear fluxes. Explicitly, the 1D NPD difference is

$$
\left\langle\Delta \rho_{1}(Q)\right\rangle=\left.\int \mathrm{d} \mathbf{q} \Delta \rho\left(\mathbf{q}, Q_{1}\right)\right|_{Q_{1}=Q}
$$

and the corresponding NPDs are

$$
\begin{array}{r}
\left\langle\rho_{1}\left(Q_{1}, t\right)\right\rangle_{\mathrm{R}}=\left\langle\rho_{1}\left(Q_{1}\right)\right\rangle_{\mathrm{R}}+\left\langle\Delta \rho_{1}\left(Q_{1}\right)\right\rangle \sin ^{2}(\pi t / \tau) \\
\left\langle\rho_{1}\left(Q_{1}, t\right)\right\rangle_{\mathrm{P}}=\left\langle\rho_{1}\left(Q_{1}\right)\right\rangle_{\mathrm{P}}-\left\langle\Delta \rho_{1}\left(Q_{1}\right)\right\rangle \sin ^{2}(\pi t / \tau)
\end{array}
$$

Expressions analogous to those in eqn (61) are derived for the core electrons of $\mathrm{B}_{4}$ in Section 4 .

For the applications to $\mathrm{B}_{4}$ and SVB in Sections 4 and 5 we choose cylindrical coordinates and evaluate the angular valence EFDs according to eqn (43) as

$$
\begin{aligned}
& \left\langle j_{\mathrm{e}, \mathrm{NCM} ; 1 \mathrm{D}, \varphi}(\varphi, t)\right\rangle_{\mathrm{R}}=\left\langle j_{\mathrm{e}, \mathrm{NCM} ; 1 \mathrm{D}, \varphi}\left(\varphi_{0}, t\right)\right\rangle_{\mathrm{R}} \\
& -\frac{\partial\left\langle P_{\mathrm{e}, \mathrm{NCM} ; 1 \mathrm{D}}\left(\varphi, \varphi_{0}, t\right)\right\rangle_{\mathrm{R}}}{\partial t}
\end{aligned}
$$

$$
\begin{aligned}
& \left\langle j_{\mathrm{e}, \mathrm{NCM} ; 1 \mathrm{D}, \varphi}(\varphi, t)\right\rangle_{\mathrm{P}}=\left\langle j_{\mathrm{e}, \mathrm{NCM} ; 1 \mathrm{D}, \varphi}\left(\varphi_{0}, t\right)\right\rangle_{\mathrm{P}} \\
& -\frac{\partial\left\langle P_{\mathrm{e}, \mathrm{NCM} ; 1 \mathrm{D}}\left(\varphi, \varphi_{0}, t\right)\right\rangle_{\mathrm{P}}}{\partial t}
\end{aligned}
$$

where $\left\langle P_{\mathrm{e}, \mathrm{NCM} ; 1 \mathrm{D}}\left(\varphi, \varphi_{0}, t\right)\right\rangle_{\mathrm{R}}$ and $\left\langle P_{\mathrm{e}, \mathrm{NCM} ; 1 \mathrm{D}}\left(\varphi, \varphi_{0}, t\right)\right\rangle_{\mathrm{P}}$ stand for the populations of valence electrons in the cylindrical sector bounded by half planes at $\varphi_{0}$ and $\varphi$ that result from the initial reactant and product states. We exploit the molecular symmetry, which allows us to choose the reference angle $\varphi_{0}$ so that $\left\langle j_{\mathrm{e}, \mathrm{NCM} ; 1 \mathrm{D}, \varphi}\left(\varphi_{0}, t\right)\right\rangle_{\mathrm{R}}=0$ or $\left\langle j_{\mathrm{e}, \mathrm{NCM} ; 1 \mathrm{D}, \varphi}\left(\varphi_{0}, t\right)\right\rangle_{\mathrm{P}}=0$. Inserting the results for the corresponding reduced densities into eqn (62), we obtain

$$
\begin{aligned}
& \left\langle j_{\mathrm{e}, \mathrm{NCM} ; 1 \mathrm{D}, \varphi}(\varphi, t)\right\rangle_{\mathrm{R}} \\
& \quad=-\int_{\varphi_{0}}^{\varphi} \mathrm{d} \varphi^{\prime} \int_{0}^{\infty} \mathrm{d} r r \int_{-\infty}^{\infty} \mathrm{d} z\left\langle\Delta \rho_{\mathrm{e}}\left(r, \varphi^{\prime}, z\right)\right\rangle(\pi / \tau) \sin (2 \pi t / \tau)
\end{aligned}
$$

$$
\begin{aligned}
& \left\langle j_{\mathrm{e}, \mathrm{NCM} ; 1 \mathrm{D}, \varphi}(\varphi, t)\right\rangle_{\mathrm{P}} \\
& \quad=\int_{\varphi_{0}}^{\varphi} \mathrm{d} \varphi^{\prime} \int_{0}^{\infty} \mathrm{d} r r \int_{-\infty}^{\infty} \mathrm{d} z\left\langle\Delta \rho_{\mathrm{e}}\left(r, \varphi^{\prime}, z\right)\right\rangle(\pi / \tau) \sin (2 \pi t / \tau)
\end{aligned}
$$

The angular flux is thus a product of an angular factor times a periodic function of the time, with period $\tau$. Likewise, from eqn (46) and (61) we get

$$
\begin{gathered}
\left\langle j_{1}\left(Q_{1}, t\right)\right\rangle_{\mathrm{R}}=-\int_{Q_{10}}^{Q_{1}} \mathrm{~d} Q_{1}{ }^{\prime}\left\langle\Delta \rho_{1}\left(Q_{1}{ }^{\prime}\right)\right\rangle(\pi / \tau) \sin (2 \pi t / \tau) \\
\left\langle j_{1}\left(Q_{1}, t\right)\right\rangle_{\mathrm{P}}=\int_{Q_{10}}^{Q_{1}} \mathrm{~d} Q_{1}{ }^{\prime}\left\langle\Delta \rho_{1}\left(Q_{1}{ }^{\prime}\right)\right\rangle(\pi / \tau) \sin (2 \pi t / \tau)
\end{gathered}
$$

where the reference value $Q_{10}$ is chosen such that $\left\langle j_{1}\left(Q_{10}, t\right)\right\rangle_{\mathrm{R}}=0$ or $\left\langle j_{1}\left(Q_{10}, t\right)\right\rangle_{\mathrm{P}}=0$. The analogues of eqn (64) are derived in Section 4 for the core electrons of $\mathrm{B}_{4}$. In summary, all of the reduced electronic and nuclear fluxes associated with the special internal nuclear coordinate $Q_{1}$ consist of a simple product of a function of the coordinates and the same periodic temporal factor. Analogous expressions hold for the reduced fluxes associated with any other internal coordinate $Q_{\alpha}$ (see Appendix A). Thus, in the lowest tunnelling doublet the electronic and nuclear fluxes are always synchronous.

We note that our definition of the main directions of the nuclear fluxes is similar to that of Makri and Miller's choice of the tunnelling path as the "straight line that connects the initial and final state in the shortest possible way". ${ }^{74}$ In fact, in the limit of very massive nuclei, the two definitions agree. Nevertheless, we emphasize that the "main directions of the fluxes during tunneling" and the "tunneling path" are different concepts and serve different purposes. Thus, "tunneling paths" are often defined as paths that allow efficient semiclassical evaluations of tunnelling splittings, times and rates (see eqn (52)). ${ }^{74-77}$ For that purpose, curvilinear tunnelling paths may be more appropriate than straight ones. ${ }^{75-79}$ In contrast, the present "main directions" are properties of the fluxes. The applications below reveal different main directions for nuclear and electronic fluxes. 
These differences confirm that one cannot necessarily identify the main directions of the fluxes with the tunnelling path.

\subsection{The triumphs and the defeat of the Born-Oppenheimer approximation}

The development of the theory is thus far based upon the formally exact solution of the Schrödinger equation (eqn (10b)), except where the customary approximations (i)-(iii) summarized at the end of Section 2.3 are invoked. We emphasize that all the results which are derived until this juncture (e.g., the synchronicity of electronic and nuclear fluxes during coherent tunnelling) are rigorous analytical results. In particular, they do not depend on the Born-Oppenheimer approximation (BOA). ${ }^{80,81}$ We would rather not refer to such results as "non-Born-Oppenheimer", since this latter phrase assigns the BOA the role of a reference, which it does not merit. Instead, we call them "without BornOppenheimer" results. The dogma that synchronicity of the CENFs during tunnelling is a consequence of the $\mathrm{BOA}^{82}$ is simply wrong. Nevertheless, in the treatment of electronically adiabatic processes, it is common practice to invoke the BOA, in which the internal wave function is taken to be a simple product

$$
\Psi(\mathbf{q}, \mathbf{Q}, t) \simeq \Psi_{\mathrm{BOA}}(\mathbf{q}, \mathbf{Q}, t)=\Phi(\mathbf{q} ; \mathbf{Q}) \chi(\mathbf{Q}, t)
$$

where $\Phi(\mathbf{q} ; \mathbf{Q})$ is an eigenfunction of the electronic Hamiltonian

$$
H_{\mathrm{e}} \Phi(\mathbf{q}, \mathbf{Q})=E(\mathbf{Q}) \Phi(\mathbf{q} ; \mathbf{Q})
$$

and $\chi(\mathbf{Q}, t)$ satisfies the nuclear Schrödinger equation

$$
i \hbar \frac{\partial \chi(\mathbf{Q}, t)}{\partial t}=\left[T_{\mathrm{n}}+V(\mathbf{Q})\right] \chi(\mathbf{Q}, t)
$$

where $V(\mathbf{Q}) \equiv E(\mathbf{Q})$ is the effective potential energy for nuclear motion. For linear and nonlinear molecules, two or three of the internal nuclear coordinates, say the last ones $\left(\boldsymbol{Q}_{3 N_{\mathrm{n}}-5}\right), \boldsymbol{Q}_{3 N_{\mathrm{n}}-4}$, $\boldsymbol{Q}_{3 N_{\mathrm{n}}-3}$ describe rotations, or orientations. Though the potential energy does not depend on these coordinates, we nevertheless keep the general notation $V(\mathbf{Q})$ to facilitate descriptions of molecules in external fields, where $V(\mathbf{Q})$ includes interactions with those fields. The electronic eigenfunction $\Phi(\mathbf{q} ; \mathbf{Q})$ depends parametrically on the internal nuclear coordinates $\mathbf{Q}$. For non-degenerate electronic states, in particular the electronic ground state, $\Phi(\mathbf{q} ; \mathbf{Q})$ is real. (We note that for systems having degenerate electronic excited states, one can prepare the system in a complex superposition of such states and therefore compute a non-zero EFD. ${ }^{23-25}$ )

The stationary nuclear energy states satisfy the eigenvalue equation

$$
\left[T_{\mathrm{n}}+V(\mathbf{Q})\right] \chi_{\nu}(\mathbf{Q})=E_{\nu} \chi_{\nu}(\mathbf{Q})
$$

whose solution yields the eigenfunctions $\chi_{\nu}(\mathbf{Q})$ and eigenenergies $E_{\nu}$ labeled by nuclear quantum numbers $\nu$. The nuclear wavefunction can be expressed in terms of these nuclear eigenfunctions by

$$
\chi(\mathbf{Q}, t)=\sum_{\nu} c_{\nu} \exp \left(-i E_{\nu} t / \hbar\right) \chi_{\nu}(\mathbf{Q})
$$

where the constant coefficients are

$$
c_{\nu}=\int \mathrm{d} \mathbf{Q} \chi_{\nu}(\mathbf{Q}) \chi(\mathbf{Q}, 0)
$$

Eqn (68) and (69) are analogous to eqn (15) and (16). We note that the nuclear wavefunction is generally complex.

Triumphs of the BOA. On one hand the BOA possesses virtues that are documented in myriads of applications, many of which are ensconced in the standard textbooks on quantum chemistry. In many instances the BOA provides excellent approximations to time-independent properties, which can be calculated from the BOA energy eigenvalues $E_{\nu}$ (see eqn (67)) and the BOA eigenfunctions $\Phi(\mathbf{q} ; \mathbf{Q}) \chi_{\nu}(\mathbf{Q})$ (i.e., the product of the electronic energy eigenfunction and a nuclear eigenfunction, usually that for the rotational-vibrational ground state $\left.\chi_{0}(\mathbf{Q})\right)$. For example, in the BOA the splitting of the lowest tunnelling doublet is $\Delta E=E_{1}-E_{0} \simeq$ $\Delta E_{\mathrm{BOA}}=E_{\nu=1}-E_{\nu=0}$; the related tunnelling time in the $\mathrm{BOA}$ is $\tau_{\mathrm{BOA}}=$ $h / \Delta E_{\mathrm{BOA}}$; the mean energy of the doublet is $\bar{E}=\left(E_{\nu=1}+E_{\nu=0}\right) / 2$.

Furthermore, for many systems, the nuclear Schrödinger equation (eqn (66b)) yields excellent approximations to time-dependent nuclear properties, which are expressed as expectation values of the relevant nuclear observables with respect to $\chi(\mathbf{Q}, t)$. In particular, the probability density for observing the internal nuclear coordinate $Q_{\alpha}$ in the range $\mathrm{d} Q$ about the point $Q$ is given by

$$
\begin{aligned}
& \left\langle\rho_{\alpha}(Q, t)\right\rangle_{\mathrm{BOA}} \\
& \quad=\int \mathrm{d} \mathbf{q} \prod_{\beta \neq \alpha} \int \mathrm{d} Q_{\beta} \Phi(\mathbf{q} ; \mathbf{Q}) \chi^{*}(\mathbf{Q}, t) \delta\left(Q-Q_{\alpha}\right) \Phi(\mathbf{q} ; \mathbf{Q}) \chi(\mathbf{Q}, t) \\
& \quad=\prod_{\beta \neq \alpha} \int \mathrm{d} Q_{\beta}|\chi(\mathbf{Q}, t)|_{Q_{\alpha}=Q}^{2}
\end{aligned}
$$

and the corresponding flux density

$$
\begin{aligned}
& \left\langle j_{\alpha}(Q, t)\right\rangle_{\mathrm{BOA}}=\int \mathrm{d} \mathbf{q} \prod_{\beta \neq \alpha} \int \mathrm{d} Q_{\beta} \frac{\hbar}{2 i \mu_{\alpha}}\left[\Phi(\mathbf{q} ; \mathbf{Q}) \chi^{*}(\mathbf{Q}, t)\left(\partial / \partial Q_{\alpha}\right)\right. \\
& \left.\left.\times \Phi(\mathbf{q} ; \mathbf{Q}) \chi(\mathbf{Q}, t)-\Phi(\mathbf{q} ; \mathbf{Q}) \chi(\mathbf{Q}, t) \partial / \partial Q_{\alpha}\right) \Phi(\mathbf{q} ; \mathbf{Q}) \chi^{*}(\mathbf{Q}, t)\right]_{Q_{\alpha}=Q} \\
& =\frac{\hbar}{2 i \mu_{\alpha}} \prod_{\beta \neq \alpha} \int \mathrm{d} Q_{\beta}\left[\chi^{*}(\mathbf{Q}, t) \frac{\partial \chi(\mathbf{Q}, t)}{\partial Q_{\alpha}}-\chi(\mathbf{Q}, t) \frac{\partial \chi^{*}(\mathbf{Q}, t)}{\partial Q_{\alpha}}\right]_{Q_{\alpha}=Q}
\end{aligned}
$$

Defeat of the BOA. On the other hand, the BOA fails if one attempts to compute the EFD for the electronic ground state using the BOA wave function (eqn (65)). Direct substitution of $\Psi_{\text {BOA }}(\mathbf{q}, \mathbf{Q}, t)$ into eqn $(29 b)$ yields

$$
\begin{aligned}
& \left\langle\mathbf{j}_{\mathrm{e}, \mathrm{NCM}}(\mathbf{x}, t)\right\rangle_{\mathrm{BOA}} \\
& \quad=N_{\mathrm{e}} \frac{\hbar}{2 i m_{\mathrm{e}}} \int \mathrm{d} \mathbf{Q}|\chi(\mathbf{Q}, t)|^{2} \prod_{j \neq 1}^{N_{\mathrm{e}}} \int \mathrm{d} \mathbf{q}_{j}\left[\Phi_{0} \nabla_{\mathbf{q}_{1}} \Phi_{0}-\Phi_{0} \nabla_{\mathbf{q}_{1}} \Phi_{0}\right]_{\mathbf{q}_{1}=\mathbf{x}}=0
\end{aligned}
$$

The right member of eqn (71) vanishes because the electronic ground-state energy eigenfunction $\Phi=\Phi_{0}$ is real. A consequence of eqn (71) is that the electronic continuity equation (eqn (31a)) becomes, in the BOA,

$$
\partial\left\langle\rho_{\mathrm{e}, \mathrm{NCM}}(\mathbf{x}, t)\right\rangle_{\mathrm{BOA}} / \partial t=0
$$


where

$$
\left\langle\rho_{\mathrm{e}, \mathrm{NCM}}(\mathbf{x}, t)\right\rangle_{\mathrm{BOA}}=N_{\mathrm{e}} \int \mathrm{d} \mathbf{Q}|\chi(\mathbf{Q}, t)|^{2} \prod_{j \neq 1}^{N_{\mathrm{e}}} \int \mathrm{d} \mathbf{q}_{j}\left[\Phi_{0}(\mathbf{q}, \mathbf{Q})\right]_{\mathbf{q}_{1}=\mathbf{x}}^{2}
$$

Eqn (72) is obviously inconsistent with eqn (73) and, to state the matter bluntly, indicates a massive defect in the BOA. In order to bypass eqn (72) and yet exploit the vast infrastructure of quantum chemistry and dynamics that is founded on the BOA, we follow two pathways.

Beyond the BOA. On the first we simply postulate that eqn (31a), which follows rigorously from the Schrödinger equation (see Appendix A), continues to hold. Hence, we define a "beyond BOA" (bBOA) flux density such that

$$
\partial\left\langle\rho_{\mathrm{e}, \mathrm{NCM}}(\mathbf{x}, t)\right\rangle_{\mathrm{BOA}} / \partial t+\nabla_{\mathbf{x}} \cdot\left\langle\mathbf{j}_{\mathrm{e}, \mathrm{NCM}}(\mathbf{x}, t)\right\rangle_{\mathrm{bBOA}}=0
$$

By reducing this $3 \mathrm{D}$ continuity equation to one dimension we can utilize such expressions as given by eqn (36), (39), (43), (62) and (63) with the exact EPD $\rho_{\mathrm{e}}$ replaced by its BOA counterpart, which we know to be quite accurate. In this fashion we are able to compute the reduced electronic fluxes beyond BOA, while using the BOA results for the electronic densities.

The bBOA scheme yields CENFs in excellent agreement with those produced by highly accurate simulations of the prototype. ${ }^{1}$ At present this simple system is the only one that allows comparison of bBOA and numerically "exact" fluxes. Extended comparisons are presented in Section 3.

Coupled-channels theory. The second pathway is rather more general than the first, in that it aims for the 3D EFD itself. It is based on the idea that the principal contribution to the EFD is due to the quasi-static movement of electrons that are associated with the nuclei. Here we follow a "quasi-classical" route $^{48}$ to the general expressions for the EFD. Thus, rather than start from the strictly classical expression for the EFD (eqn (28b)), we begin by supposing that effective EPDs can be assigned to the nuclei and define a quasi-classical EFD in the laboratory frame by

$$
\mathbf{j}_{\mathrm{e}, \mathrm{L}}(\mathbf{x}, t)=\sum_{a=1}^{N_{\mathrm{n}}} \rho_{a}(\mathbf{x} ; \mathbf{R}(t)) \dot{\mathbf{R}}_{a}(t)
$$

where $\mathbf{R}(t)$ stands for the nuclear configuration and $\dot{\mathbf{R}}_{a}(t)$ for the velocity of nucleus $a$. The EPDs associated with the nuclei, determined by the LCAO-MO method, are given by

$$
\rho_{a}(\mathbf{x} ; \mathbf{R})=\sum_{i} n_{i} \psi_{a}^{(i)}(\mathbf{x} ; \mathbf{R}) \phi_{i}(\mathbf{x} ; \mathbf{R})
$$

where the summation on $i$ runs over the occupied MOs, $n_{i}$ is the number of electrons in the $i$ th $\mathrm{MO}$, and

$$
\begin{gathered}
\psi_{a}^{(i)}(\mathbf{x} ; \mathbf{R})=\sum_{l_{\alpha}} c_{a, l_{\alpha}}^{(i)} \varphi_{l_{\alpha}}^{(a)}(\mathbf{x} ; \mathbf{R}) \\
\phi_{i}(\mathbf{x} ; \mathbf{R})=\sum_{a} \psi_{a}^{(i)}(\mathbf{x} ; \mathbf{R})
\end{gathered}
$$

That is, $\psi_{a}^{(i)}$ is the component of the $i$ th MO attributed to atomic orbitals $\varphi_{l_{\alpha}}^{(a)}$ centered on nucleus $a$. We refer to the formula in eqn (75) as "quasi-classical” because the quantum dynamical character of the electrons in the BOA is implicit in the EPDs. Only the explicit classical dynamics of the nuclei remains. The EFD, which entails the quantum nature of the nuclei, is then given by the expectation value of the operator corresponding to $\mathbf{j}_{\mathrm{e}, \mathrm{L}}(\mathbf{x}, t)$, which can be computed from the BOA nuclear wave packet $\chi(\mathbf{Q}, t)$ alone. (Note that the meaning of the term "quasi-classical" here differs from that of the same term used in other contexts to mean "semi-classical".)

In the case of the one-electron diatomic molecule $\mathrm{AB}$, the coupled-channels theory gives ${ }^{46,48}$

$$
\begin{aligned}
\left\langle\mathbf{j}_{\mathrm{e}, \mathrm{NCM}}(\mathbf{x}, t)\right\rangle_{\mathrm{CC}}= & \int \mathrm{d} \mathbf{Q}_{1}\left[M_{b} \Delta_{a}\left(\mathbf{x} ; \mathbf{Q}_{1}\right) / M_{\mathrm{n}}\right. \\
& \left.-M_{a} \Delta_{b}\left(\mathbf{x} ; \mathbf{Q}_{1}\right) / M_{\mathrm{n}}\right]\left\langle\mathbf{j}_{a, b}\left(\mathbf{Q}_{1}, t\right)\right\rangle_{\mathrm{BOA}}
\end{aligned}
$$

where

$$
\begin{aligned}
& \left\langle\mathbf{j}_{a, b}\left(\mathbf{Q}_{1}, t\right)\right\rangle_{\mathrm{BOA}} \\
& =\frac{\hbar}{2 \mu_{a b} i}\left[\chi^{*}\left(\mathbf{Q}_{1}, t\right) \nabla_{\mathbf{Q}_{1}} \chi\left(\mathbf{Q}_{1}, t\right)-\chi\left(\mathbf{Q}_{1}, t\right) \nabla_{\mathbf{Q}_{1}} \chi^{*}\left(\mathbf{Q}_{1}, t\right)\right]
\end{aligned}
$$

is the flux density of nucleus $a$ with respect to nucleus $b$, which is determined accurately in the BOA (see eqn (70)). [Note that the term "coupled-channels" in the present context refers simply to the form of the EFD, i.e., the difference between contributions of channel $a$ (defined as "internal" atom $a$ "colliding" with nucleus $b$ ) and channel $b$ (internal atom $b$ colliding with nucleus $a$ ) to the EFD, as seen in eqn (78); this coupled-channels theory of the EFD is an approximation to the traditional quantum-dynamical description of collisions.] Recall that $\mathbf{Q}_{1}=\mathbf{R}_{a}-\mathbf{R}_{b}$ (see discussion below eqn (6)). The EPDs corresponding to the singly occupied lowest-energy MO $\phi_{1}$ are

$$
\begin{aligned}
& \Delta_{a}\left(\mathbf{x} ; \mathbf{Q}_{1}\right) \equiv \phi_{1}\left(\mathbf{x} ; \mathbf{Q}_{1}\right) \psi_{a}^{(1)}\left(\mathbf{x} ; \mathbf{Q}_{1}\right) \\
& \Delta_{b}\left(\mathbf{x} ; \mathbf{Q}_{1}\right) \equiv \phi_{1}\left(\mathbf{x} ; \mathbf{Q}_{1}\right) \psi_{b}^{(1)}\left(\mathbf{x} ; \mathbf{Q}_{1}\right)
\end{aligned}
$$

where

$$
\begin{aligned}
\phi_{1}\left(\mathbf{x} ; \mathbf{Q}_{1}\right)=\psi_{a}^{(1)}\left(\mathbf{x} ; \mathbf{Q}_{1}\right)+\psi_{b}^{(1)}\left(\mathbf{x} ; \mathbf{Q}_{1}\right) \\
\psi_{a}^{(1)}\left(\mathbf{x} ; \mathbf{Q}_{1}\right)=\sum_{l_{\alpha}} c_{a, l_{\alpha}}^{(1)} \varphi_{l_{\alpha}}^{(a)}\left(\mathbf{x} ; \mathbf{Q}_{1}\right) ; \psi_{b}^{(1)}\left(\mathbf{x} ; \mathbf{Q}_{1}\right) \\
=\sum_{l_{\alpha}} c_{b, l_{\alpha}}^{(1)} \varphi_{l_{\alpha}}^{(b)}\left(\mathbf{x} ; \mathbf{Q}_{1}\right)
\end{aligned}
$$

For the particular instance of the homonuclear diatomic, eqn (78) becomes

$$
\left\langle\mathbf{j}_{\mathrm{e}, \mathrm{NCM}}(\mathbf{x}, t)\right\rangle_{\mathrm{CC}}=\frac{1}{2} \int \mathrm{d} \mathbf{Q}_{1}\left[\Delta_{a}\left(\mathbf{x} ; \mathbf{Q}_{1}\right)-\Delta_{b}\left(\mathbf{x} ; \mathbf{Q}_{1}\right)\right]\left\langle\mathbf{j}_{a, b}\left(\mathbf{Q}_{1}, t\right)\right\rangle_{\mathrm{BOA}}
$$

The application in Section 3.1 to vibrating oriented $\mathrm{H}_{2}^{+}$utilizes the coupled-channels EFD, along with the electronic continuity equation and a scaling procedure, ${ }^{51}$ to obtain good agreement with the benchmark calculation based on the B-spline spectral method. ${ }^{50}$ 


\section{Application of the theory to the hydrogen-molecular ion}

3.1 The prototype: vibrating aligned $\mathrm{H}_{2}{ }^{+}$in the electronic ground state ${ }^{2} \Sigma_{\mathrm{g}}{ }^{+}$

This section serves two purposes: (1) to establish a highly accurate standard (to which we henceforward refer simply as "benchmark") for the testing of approximate methods, in particular "beyond BOA" and the scaled coupled-channels technique; (2) to reveal new phenomena exhibited by CENFs in the prototype.

Since alignment of the nuclei corresponds theoretically to the constraint that the nuclei move on a straight line, our model is $1 \mathrm{D}$, insofar as nuclear motion is concerned. The electron is not, of course, so constrained. In this special case eqn (26) reduces to

$$
\begin{gathered}
H_{\mathrm{e}}=-\frac{\hbar^{2}}{2 m_{\mathrm{e}}} \nabla_{\mathbf{r}}^{2}+\frac{e^{2}}{4 \pi \varepsilon_{0}}\left[\frac{1}{R}-\frac{1}{\left|\mathbf{r}-R \mathbf{e}_{z} / 2\right|}-\frac{1}{\left|\mathbf{r}+R \mathbf{e}_{z} / 2\right|}\right] \\
T_{\mathrm{n}}=-\frac{\hbar^{2}}{2 \mu} \frac{\partial^{2}}{\partial R^{2}}
\end{gathered}
$$

where $\mathbf{r} \equiv \mathbf{q}, R \equiv Q=Z_{a}-Z_{b}, \mu=M_{\mathrm{p}} / 2$, and $M_{\mathrm{p}}$ is the mass of the proton. According to eqn (15) the exact wave function is

$$
\Psi(\mathbf{r}, R, t)=\sum_{n} c_{n} \exp \left(-i E_{n} t / \hbar\right) \psi_{n}(\mathbf{r}, R)
$$

where the vibronic energy eigenfunctions satisfy

$$
\left(H_{\mathrm{e}}+T_{\mathrm{n}}\right) \psi_{n}(\mathbf{r}, R)=E_{n} \psi_{n}(\mathbf{r}, R)
$$

We utilize the B-spline spectral method ${ }^{50}$ to determine the eigenstates. For this purpose it is convenient to employ a spherical coordinate system whose origin coincides with the NCM (Fig. 1). The polar angle $\theta$ is measured from the positive $z$-axis. Nucleus $a$ lies at $R \mathbf{e}_{z} / 2$ and nucleus $b$ at $-R \mathbf{e}_{z} / 2$. Because of the cylindrical symmetry of the state ${ }^{2} \Sigma_{\mathrm{g}}{ }^{+}$the vibronic eigenfunctions depend only on $r, \theta$, and $R$. The initial states can be prepared by exposure of $\mathrm{H}_{2}$ in the electronic ground state to short, intense, near-infrared laser pulses. ${ }^{83}$ For simplicity it is taken here to be the vibronic ground-state eigenfunction with the nuclear coordinate $R$ displaced arbitrarily by $\bar{R}$ (i.e., $\left.\Psi(r, \theta, R, 0)=\psi_{0}(r, \theta, R-\bar{R})\right)$. For the results to be presented here, we set $\bar{R}=2.0 \mathrm{a}_{0}$.

The general expression in eqn (29b) for the EFD becomes

$$
\left\langle\mathbf{j}_{\mathrm{e}, \mathrm{NCM}}(\mathbf{x}, t)\right\rangle=\frac{\hbar}{2 i m_{\mathrm{e}}} \int_{0}^{\infty} \mathrm{d} R\left[\Psi^{*} \nabla_{\mathbf{r}} \Psi-\Psi \nabla_{\mathbf{r}} \Psi^{*}\right]_{\mathbf{r}=\mathbf{x}}
$$

Exploiting the symmetry of the wave function, we can rewrite eqn (86a) as ${ }^{72}$

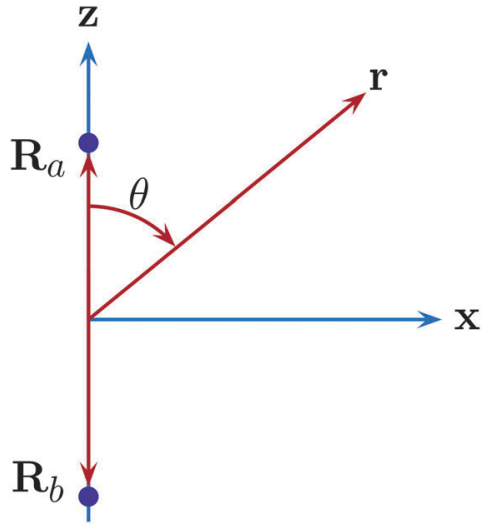

Fig. 1 Schematic view of $\mathrm{H}_{2}{ }^{+}$in direction of $y$ axis with nuclei on $z$ axis at positions $\mathbf{R}_{a}$ and $\mathbf{R}_{b}$ with respect to $\mathrm{NCM}$ at origin. Spherical coordinates of electron with respect to NCM are $r, \theta$, and $\varphi$ (not shown)

where the second line of eqn (86b) defines the radial ( $\mathbf{e}_{\perp}$ stands for the unit vector perpendicular to the $z$ axis) and $z$-components of the electronic flux density in cylindrical coordinates. The flux density of nucleus $a$ at observation point $R^{\prime}$ relative to the NCM is

$$
\begin{aligned}
\left\langle j_{a, \mathrm{NCM}}\left(R^{\prime}, t\right)\right\rangle= & \frac{\hbar}{2 i \mu} \int_{0}^{2 \pi} \mathrm{d} \phi \int_{0}^{\pi} \mathrm{d} \theta \sin \theta \int_{0}^{\infty} \mathrm{d} r r^{2} \\
& \times\left[\Psi^{*}(r, \theta, R, t) \frac{\partial}{\partial R} \Psi(r, \theta, R, t)-\text { c.c. }\right]_{R=2 R^{\prime}}
\end{aligned}
$$

Henceforth we refer to $\left\langle j_{a, \mathrm{NCM}}\left(R^{\prime}, t\right)\right\rangle$ as the NFD, or simply nuclear flux, recalling that for the $1 \mathrm{D}$ model flux density and flux are the same.

Fig. 2 exhibits plots of the NFD versus time at several points of observation on the positive $z$-axis. Note that at $z=0.5 \mathrm{a}_{0}$ and $z=2.5 \mathrm{a}_{0}$ the NFD is negligible, since the wave function itself is, for this particular state, negligible outside the range $0.5 \mathrm{a}_{0} \leq$ $z \leq 2.5 \mathrm{a}_{0}$. Negative and positive fluxes correspond to contraction and expansion of the bond. Fig. 2 indicates that the bond undergoes one expansion-contraction cycle during the first $22.3 \mathrm{fs}$. This cyclic process repeats itself until $t \simeq 70 \mathrm{fs}$, though scrutiny reveals that the relative magnitudes and phases of the fluxes at the three points shift slightly with increasing time. Beyond $t \simeq 70$ fs a chaotic period ensues, during which the magnitudes and phases appear to get thoroughly shuffled, presumably as a consequence of the spreading of the wave packet and its consequent self-interference. After about $t \simeq 225 \mathrm{fs}$ the desultory character of the NFDs declines relatively quickly,

$$
\begin{aligned}
\left\langle\mathbf{j}_{\mathrm{e}, \mathrm{NCM}}(\mathbf{x}, t)\right\rangle= & \frac{\hbar}{2 i m_{\mathrm{e}}} \int_{0}^{\infty} \mathrm{d} R\left[\Psi^{*}(r, \theta, R, t)\left(\sin \theta \frac{\partial}{\partial r}+\frac{\cos \theta}{r} \frac{\partial}{\partial r}\right) \Psi(r, \theta, R, t)-\text { c.c. }\right]_{\mathbf{r}=\mathbf{x}} \mathbf{e}_{\perp} \\
& \quad+\frac{\hbar}{2 i m_{\mathrm{e}}} \int_{0}^{\infty} \mathrm{d} R\left[\Psi^{*}(r, \theta, R, t)\left(\cos \theta \frac{\partial}{\partial r}-\frac{\sin \theta}{r} \frac{\partial}{\partial r}\right) \Psi(r, \theta, R, t)-\text { c.c. }\right]_{\mathbf{r}=\mathbf{x}} \mathbf{e}_{z} \\
= & \left\langle j_{\mathrm{e}, \mathrm{NCM}, \perp}(\mathbf{x}, t)\right\rangle \mathbf{e}_{\perp}+\left\langle j_{\mathrm{e}, \mathrm{NCM}, z}(\mathbf{x}, t)\right\rangle \mathbf{e}_{z}
\end{aligned}
$$



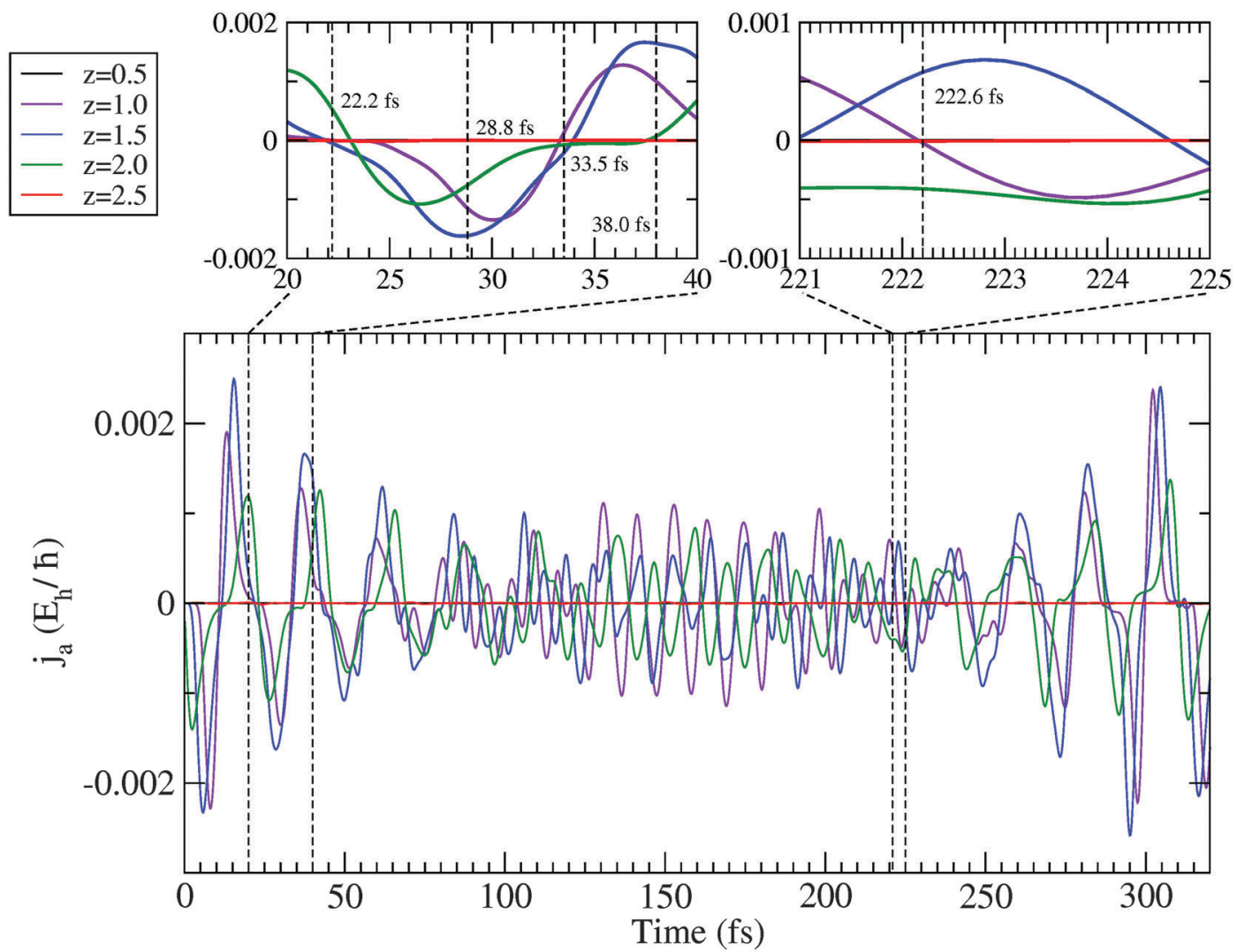

until at $t \simeq 300$ fs the pattern of the fluxes essentially matches that observed at $t \simeq 0$. The wave packet appears to have recovered its initial compact form (or global synchronicity). Scrutinous examination shows that, though the wave packet has been completely reconstructed, it is out of phase with the initial packet by $\pi$. Since the time at which the packet comes into phase with the initial packet (i.e., the revival time $T_{\text {rev }}$ ) is about $600 \mathrm{fs}$, the observed reconstruction at $300 \mathrm{fs}$ actually corresponds to the half-revival time $T_{\text {rev }} / 2$, which is also observed experimentally. ${ }^{84}$ Indeed, it can be shown that reconstructions of the wave packet generally occur at times $t=p T_{\text {rev }} / q$, where $p$ and $q$ are integers and $p / q$ is irreducible. ${ }^{85}$ Such fractional revivals are also seen experimentally for $\mathrm{D}_{2}{ }^{+86}$ and for $\mathrm{Br}_{2} \cdot{ }^{87}$

The left inset in Fig. 2, which displays the plots on a finer scale in the range from 20 to $40 \mathrm{fs}$, shows that the NFDs evolve nearly in phase. In contrast, the right inset shows that the nuclear fluxes are out of phase in the range from 221 to 225 fs. Such strong shifts in the relative phases of the NFDs have been reported recently for $\mathrm{D}_{2}{ }^{+}$and $\mathrm{Na}_{2} \cdot{ }^{8}$

Fig. 3 exhibits plots of the $z$-component of the EFD versus time at the same points of observation used for the NFDs. Because of the symmetry of the ${ }^{2} \Sigma_{\mathrm{g}}{ }^{+}$state, the EFDs observed at $-0.5 \mathrm{a}_{0}$, etc., are the negatives of those observed at $0.5 \mathrm{a}_{0}$, etc. The same is true of the flux of nucleus $b$ with respect to the NCM. We note that for the first $70 \mathrm{fs}$, the pattern of the EFDs mirrors that of their nuclear counterparts (i.e., as one expects intuitively, the EFDs are synchronous with the NFDs). However, in the period from 70 to 225 fs the EFDs remain in phase, whereas the phases of the NFDs are jumbled. The absence of strong dephasing of the EFDs compared with the NFDs is a consequence of the relatively broad spread of the EPD compared with the nuclear density.

The right inset in Fig. 3, which displays the EFDs in the range from 221-225 fs, is especially interesting, as it reveals for the first time multiple reversals in the direction of the EFD. The phenomenon is reminiscent of the previous discovery of multiple reversals in the direction of the NFD, an effect which has been coined "quantum accordion". ${ }^{8}$ We interpret this observation to indicate that, shortly before the half-revival time, the electron reacts to the highly inhomogeneous distribution of the nuclear density.

Fig. 4 shows vector plots of $\left\langle j_{\mathrm{e}, \mathrm{NCM}, \rho}(\mathbf{x}, t)\right\rangle \mathbf{e}_{\perp}+\left\langle j_{\mathrm{e}, \mathrm{NCM}, z}(\mathbf{x}, t)\right\rangle \mathbf{e}_{z}$ at several times within the short-time range shown in the left insets in Fig. 2 and 3. The results of the scaled coupled-channels (SCC) technique, ${ }^{51}$ which is based on the BOA, are compared with the highly accurate results (benchmark) obtained by the B-spline spectral procedure. ${ }^{50}$ We observe that when the EFDs take extreme values (i.e., at 22.2 and 38.0 fs) the SCC accords very well with the benchmark. However, to put it charitably for the SCC, we remark that when the EFDs are small, then the agreement in not so good. The disagreement is due not only to 


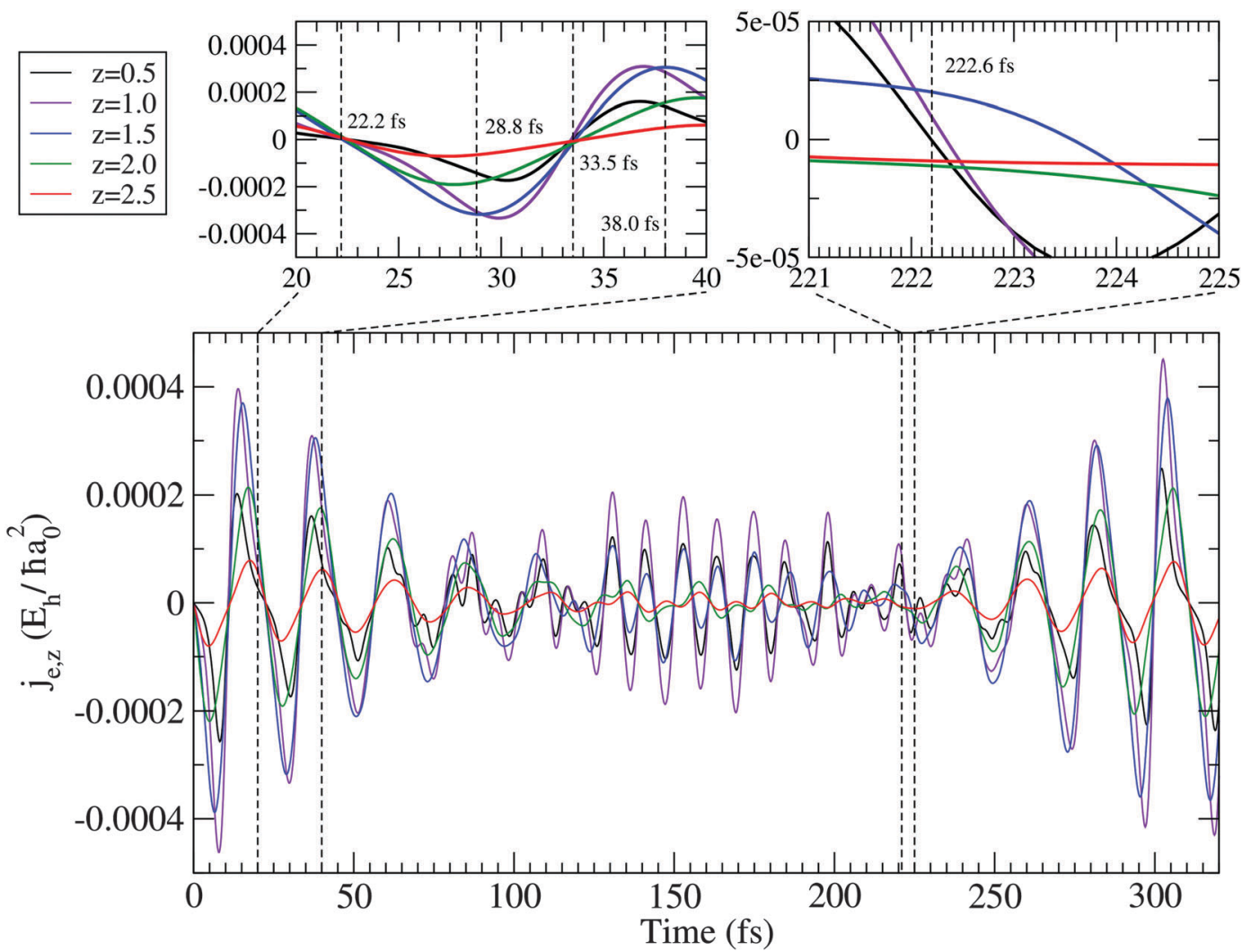

Fig. 3 Z-Component of electronic flux density versus time for prototype (vibrating, aligned $\mathrm{H}_{2}{ }^{+}$in the electronic ground state ${ }^{2} \Sigma_{\mathrm{g}}{ }^{+}$). Five curves correspond to same points of observation specified in Fig. 2. Insets focus on detail over special domains, as in Fig. 2.
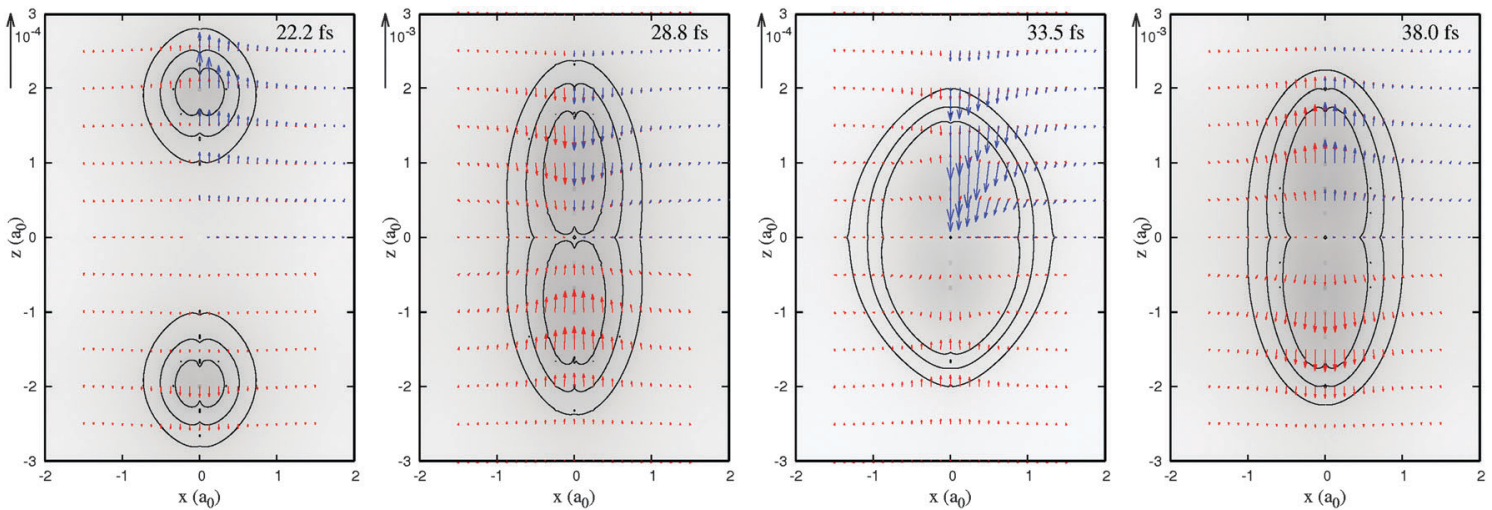

Fig. 4 Vector plots of electronic flux density for prototype (vibrating, aligned $\mathrm{H}_{2}{ }^{+}$in electronic ground state ${ }^{2} \Sigma_{\mathrm{g}}^{+}$) in $x-z$ plane at several times within interval encompassed by left insets of Fig. 2 and 3. Each plot compares scaled coupled channels result (blue) with numerically exact benchmark (red). Contour plots of benchmark electronic probability density are also shown (contour lines: $0.025,0.030$, and $0.035 \mathrm{a}_{0}{ }^{-3}$ ).

approximations inherent in the SCC, but also to differences between BOA and benchmark nuclear dynamics, which are, in turn, traceable to disparities between BOA and benchmark vibronic eigenstates. Finally in Fig. 5 we exhibit the vector plot showing several reversals in the direction of the EFD at $222.6 \mathrm{fs}$ (See the right insets in Fig. 2 and 3), which are characteristic of the "quantum accordion" effect. ${ }^{8}$

It is instructive to compare the performance "beyond BOA" (see Section 2.9, eqn (74)) with that of the benchmark in the calculation of the electronic flux through a plane normal to the internuclear $(z-)$ axis. Hence, operating upon eqn (41) with $\int_{-\infty}^{z} \mathrm{~d} z^{\prime} \int_{0}^{2 \pi} \mathrm{d} \varphi \int_{0}^{\infty} \mathrm{d} r r \ldots$, we obtain the formally exact relation $\left\langle j_{\mathrm{e}, \mathrm{NCM} ; \mathrm{ID}, z}(z, t)\right\rangle=-\partial \int_{-\infty}^{z} \mathrm{~d} z^{\prime}\left\langle\rho_{\mathrm{e}, \mathrm{NCM} ; 1 \mathrm{D}, z}\left(z^{\prime}, t\right)\right\rangle / \partial t$, which is analogous to eqn (42). If we use the BOA to compute the $\mathrm{EPD}$, then the exact relation is approximated by

$$
\left\langle j_{\mathrm{e}, \mathrm{NCM} ; 1 \mathrm{D}, z}(z, t)\right\rangle_{\mathrm{bBOA}}=-\partial \int_{-\infty}^{z} \mathrm{~d} z^{\prime}\left\langle\rho_{\mathrm{e}, \mathrm{NCM} ; \mathrm{ID}, z}\left(z^{\prime}, t\right)\right\rangle_{\mathrm{BOA}} / \partial t
$$




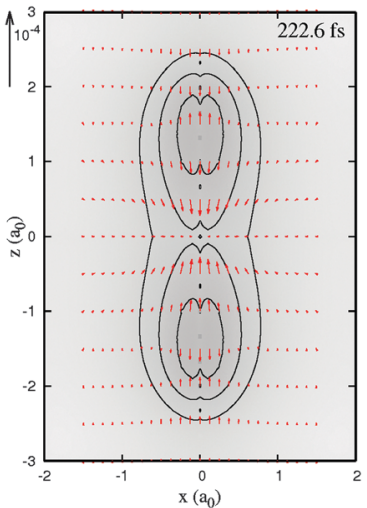

Fig. 5 Vector plot of benchmark electronic flux density for prototype (vibrating, aligned $\mathrm{H}_{2}{ }^{+}$in the electronic ground state ${ }^{2} \Sigma_{\mathrm{g}}{ }^{+}$) in $x-z$ plane at time 222.6 fs within interval encompassed by right insets of Fig. 2 and 3 . Contour plots of electronic probability density are also shown (contour lines: $0.025,0.030,0.035 \mathrm{a}_{0}{ }^{-3}$ ).

where $\left\langle\rho_{\mathrm{e}, \mathrm{NCM} ; \mathrm{ID}, z}(z, t)\right\rangle_{\mathrm{BOA}}=2 \pi \int_{0}^{\infty} \mathrm{d} r r \int_{0}^{\infty} \mathrm{d} R|\chi(R, t)|^{2}\left[\Phi_{0}(r, z ; R)\right]^{2}$ (see eqn (73)). Though the exact relation can as well be used to compute the benchmark flux, it is somewhat more convenient in practice to employ the relation $\left\langle j_{\mathrm{e}, \mathrm{NCM} ; 1 \mathrm{D}, z}(z, t)\right\rangle=$ $\int_{S} \mathbf{n d} S \cdot\left\langle\mathbf{j}_{\mathrm{e}, \mathrm{NCM}}(r, z, t)\right\rangle$, where the surface $S$ is the plane normal to the internuclear axis at $z$ and $\mathbf{n}=\mathbf{e}_{z}$ is the normal to the element $\mathrm{d} S$. From eqn (86b) we get $\left\langle j_{\mathrm{e}, \mathrm{NCM} ; 1 \mathrm{D}, z}(z, t)\right\rangle=\int_{0}^{2 \pi} \mathrm{d} \varphi \int_{0}^{\infty} \mathrm{d} r \mathbf{e}_{z}$. $\left\langle\mathbf{j}_{\mathrm{e}, \mathrm{NCM}}(r, z, t)\right\rangle=2 \pi \int_{0}^{\infty} \mathrm{d} r r\left\langle j_{\mathrm{e}, \mathrm{NCM}, z}(r, z, t)\right\rangle$. Using the bBOA and benchmark formulas derived here, we generate the contour plots displayed in Fig. 6, which indicate superb overall agreement (compare panels (a), (b) and (d)). The "beyond BOA" technique accords semi-quantitatively with the benchmark even at times when the wave packet is concentrated near a classical turning point and the flux is very small (see panel (c)). These new results confirm quantitatively the CENFs reported for the prototype in ref. 1. We are aware of no other reports of CENFs for the prototype, or any other system, by the bBOA or any other benchmark method.

\subsection{Vibrating, dissociating $\mathrm{H}_{2}{ }^{+}$as a "quantum bubble" in the} spherically symmetric electronic ground state ${ }^{2} \Sigma_{g}^{+}(J=0, M=0)$

The symmetry of this special state dictates that both the EPD and EFD are spherically symmetric. ${ }^{52}$ Therefore, according to
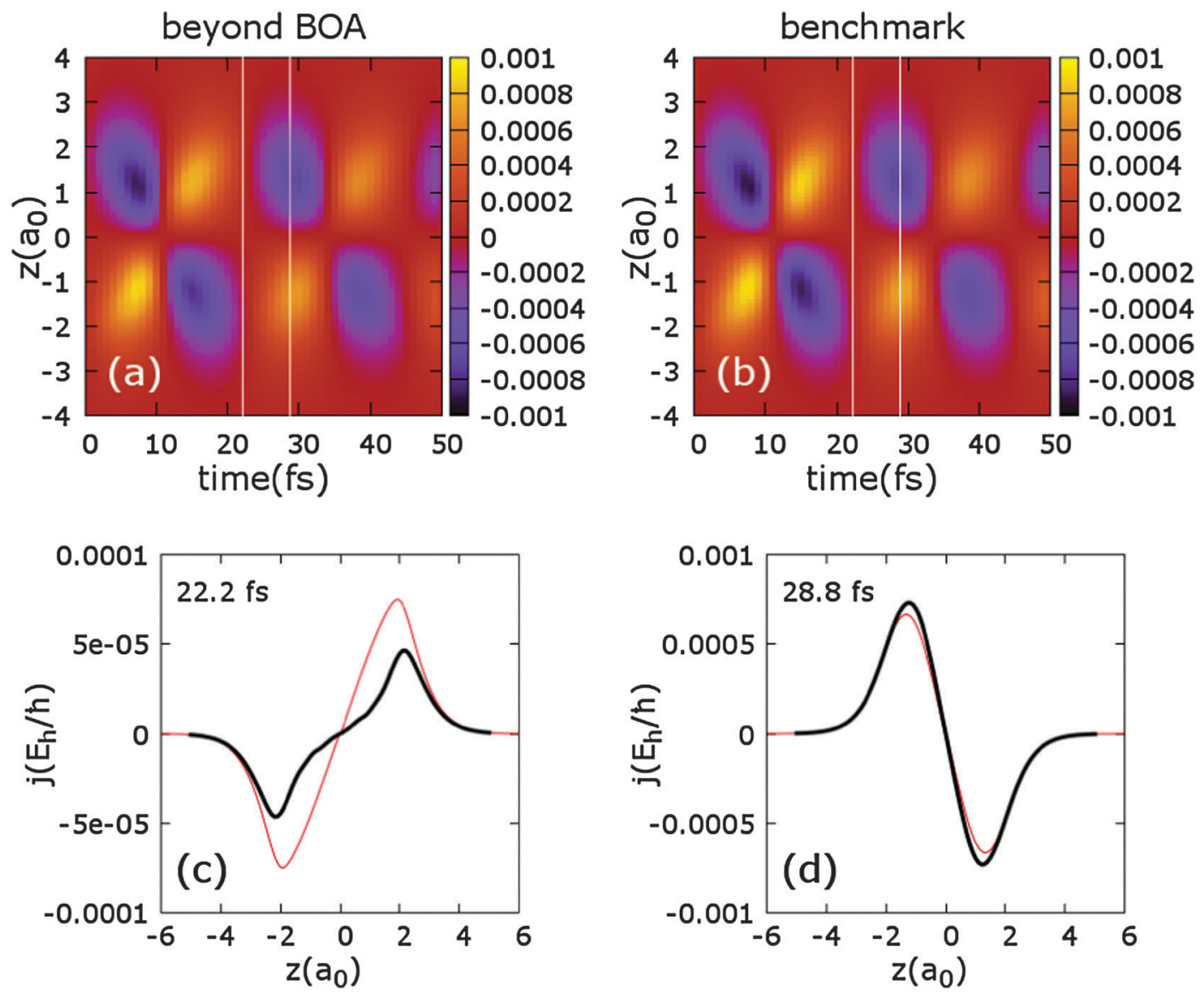

Fig. 6 Comparison of electronic flux densities (EFDs) obtained by "beyond BOA" approximation with results of benchmark for prototype (vibrating, aligned $\mathrm{H}_{2}{ }^{+}$in electronic ground state ${ }^{2} \Sigma_{\mathrm{g}}{ }^{+}$). (a and b) Contour plots showing spatial-temporal evolution of EFDs in time interval that overlaps left insets in Fig. 2 and 3. ( $c$ and d) Spatial distributions at two times [indicated by vertical lines in panels (a) and (b); beyond BOA (red line), benchmark (black line)]: $t=22.2$ fs which corresponds to a classical turning point (c); $t=28.8$ fs halfway between classical turning points (d). Note the different scales in (c) and (d). 
eqn (39), we have

$$
\left\langle j_{\mathrm{e}, \mathrm{NCM} ; 1 \mathrm{D}, r}(r, t)\right\rangle_{\mathrm{bBOA}}=-\frac{\partial}{\partial t}\left[r^{-2} \int_{0}^{r} \mathrm{~d} r^{\prime} r^{\prime 2}\left\langle\rho_{\mathrm{e}, \mathrm{NCM}}\left(r^{\prime}, t\right)\right\rangle_{\mathrm{BOA}}\right]
$$

where

$$
\begin{aligned}
\left\langle\rho_{\mathrm{e}, \mathrm{NCM}}(r, t)\right\rangle_{\mathrm{BOA}}= & (4 \pi)^{-1} \int_{0}^{\infty} \mathrm{d} R|\psi(R, t)|^{2} \\
& \times \int_{0}^{2 \pi} \mathrm{d} \Phi \int_{0}^{\pi} \mathrm{d} \theta \sin \theta\left[\Phi_{0}(r, \cos \theta ; R)\right]^{2}
\end{aligned}
$$

$\Phi_{0}$ is the ground-state eigenfunction of the electronic Hamiltonian (see eqn (66a)) and $\psi(R, t)$ is the nuclear radial wave function, which is related to the nuclear wave packet by

$$
\psi(R, t)=(4 \pi)^{1 / 2} R \chi(R, t)
$$

In eqn (90) we take the polar axis ( $z$-axis) to lie on the vector $\mathbf{r}$ from the NCM to the point of observation. The BOA flux density of nucleus $a$ with respect to the NCM, which satisfies eqn (40), can be written explicitly as

$$
\left\langle j_{a, \mathrm{NCM} ; 1 \mathrm{D}, R}(R, t)\right\rangle_{\mathrm{BOA}}=-\frac{\partial}{\partial t}\left[R^{-2} \int_{0}^{R} \mathrm{~d} R^{\prime} R^{\prime 2}\left\langle\rho_{a, \mathrm{NCM}}\left(R^{\prime}, t\right)\right\rangle_{\mathrm{BOA}}\right]
$$

where the NPD is

$$
\left\langle\rho_{a, \mathrm{NCM}}(R, t)\right\rangle_{\mathrm{BOA}}=2(4 \pi)^{-1} R^{-2}|\psi(2 R, t)|^{2}
$$

Identical formulas (with $a$ replaced by $b$ ) obtain for the nucleus $b$.

The initial wave packet is prepared through photo-ionization of $\mathrm{H}_{2}$ in the electronic ground state ${ }^{1} \Sigma_{\mathrm{g}}{ }^{+}(J=0, M=0)$, which produces $\mathrm{H}_{2}{ }^{+}$in the electronic ground state ${ }^{2} \Sigma_{\mathrm{g}}{ }^{+}(J=0, M=0)$ with the relative nuclear motion highly excited. ${ }^{53}$ Hence, the nuclear radial wave function can be written as

$$
\begin{aligned}
\psi(R, t)= & \sum_{\nu=0}^{N_{\mathrm{v}}} c_{\nu} \exp \left(-i E_{\nu} t / \hbar\right) \xi_{\nu}(R) \\
& +\sum_{\nu>N_{\mathrm{v}}} c_{\nu} \exp \left(-i E_{\nu} t / \hbar\right) \xi_{\nu}(R)=\psi_{\mathrm{v}}(R, t)+\psi_{\mathrm{d}}(R, t)
\end{aligned}
$$

where $N_{\mathrm{v}}$ is the number of vibrational bound states and the summation over states $\nu>N_{\mathrm{v}}$ constitutes a discrete representation of the continuum in terms of box-normalized eigenfunctions. We determine the radial eigenfunctions by solving the radial eigenvalue equation $\left[\left(-\hbar^{2} / 2 \mu\right) \partial^{2} / \partial R^{2}+V(R)\right] \xi_{\nu}(R)=E_{\nu} \xi_{\nu}(R)$, using the B-spline spectral procedure on the interval $0 \leq R \leq 100 \mathrm{a}_{0} .^{50}$ The constant coefficients in eqn (94) are determined by the initial wave packet: $c_{\nu}=\int_{0}^{\infty} \mathrm{d} R \xi_{\nu}(R) \psi(R, 0)$. Making use of the partitioning of the radial wave function into "vibrational" (v) and "dissociative" (d) contributions (see eqn (94)), we can express the radial probability density as

$$
|\psi(R, t)|^{2}=\left|\psi_{\mathrm{v}}(R, t)\right|^{2}+\left|\psi_{\mathrm{d}}(R, t)\right|^{2}+2 \operatorname{Re}\left[\psi_{\mathrm{v}}{ }^{*}(R, t) \psi_{\mathrm{d}}(R, t)\right]
$$

Substitution of this expression into eqn (90) and (93) gives

$$
\begin{aligned}
\left\langle\rho_{\mathrm{e}, \mathrm{NCM}}(r, t)\right\rangle_{\mathrm{BOA}} & =\left\langle\rho_{\mathrm{e}, \mathrm{NCM} ; \mathrm{v}}(r, t)\right\rangle_{\mathrm{BOA}}+\left\langle\rho_{\mathrm{e}, \mathrm{NCM} ; \mathrm{d}}(r, t)\right\rangle_{\mathrm{BOA}} \\
& +\left\langle\rho_{\mathrm{e}, \mathrm{NCM} ; \mathrm{int}}(r, t)\right\rangle_{\mathrm{BOA}}
\end{aligned}
$$

$$
\begin{aligned}
\left\langle\rho_{a, \mathrm{NCM}}(R, t)\right\rangle_{\mathrm{BOA}} & =\left\langle\rho_{a, \mathrm{NCM} ; \mathrm{v}}(R, t)\right\rangle_{\mathrm{BOA}}+\left\langle\rho_{a, \mathrm{NCM} ; \mathrm{d}}(R, t)\right\rangle_{\mathrm{BOA}} \\
& +\left\langle\rho_{a, \mathrm{NCM} ; \text { int }}(R, t)\right\rangle_{\mathrm{BOA}}
\end{aligned}
$$

where the vibrational, dissociative and "interference" (int) contributions to the probability densities are separated. Plugging these formulas into eqn (89) and (92) yields a similar decomposition of the flux densities:

$$
\begin{aligned}
\left\langle j_{\mathrm{e}, \mathrm{NCM} ; 1 \mathrm{D}, r}(r, t)\right\rangle_{\mathrm{bBOA}} & =\left\langle j_{\mathrm{e}, \mathrm{NCM} ; 1 \mathrm{D}, r, \mathrm{v}}(r, t)\right\rangle_{\mathrm{bBOA}}+\left\langle j_{\mathrm{e}, \mathrm{NCM} ; 1 \mathrm{D}, r, \mathrm{~d}}(r, t)\right\rangle_{\mathrm{bBOA}} \\
& +\left\langle j_{\mathrm{e}, \mathrm{NCM} ; 1 \mathrm{D}, r, \mathrm{int}}(r, t)\right\rangle_{\mathrm{bBOA}}
\end{aligned}
$$

$$
\begin{aligned}
\left\langle j_{a, \mathrm{NCM} ; 1 \mathrm{D}, R}(R, t)\right\rangle_{\mathrm{BOA}} & =\left\langle j_{a, \mathrm{NCM} ; 1 \mathrm{D}, R, \mathrm{v}}(R, t)\right\rangle_{\mathrm{BOA}}+\left\langle j_{a, \mathrm{NCM} ; 1 \mathrm{D}, R, \mathrm{~d}}(R, t)\right\rangle_{\mathrm{BOA}} \\
& +\left\langle j_{a, \mathrm{NCM} ; 1 \mathrm{D}, R, \mathrm{int}}(R, t)\right\rangle_{\mathrm{BOA}}
\end{aligned}
$$

Note that the EFD, as expressed by eqn (97a), must go beyond the BOA ("bBOA") in the manner described in Section 2, because in the BOA the electronic flux vanishes identically (see eqn (71)).

Fig. 7 displays contour plots of the various contributions to the EFD and NFD at two times. At the shorter time of $8 \mathrm{fs}$ the electron and the nuclei are essentially confined to a sphere of radius $6 \mathrm{a}_{0}$. One might conceive of this condition as the interference between a pulsating "bubble" and an exploding one. In other words, the bound vibrational states (pulsating) interfere destructively with the continuum states (exploding). At 80 fs multiple reversals of direction of the NFD indicate strong dephasing of the radial wave packet. In contrast, however, the EFD displays no such reversal. These observations are consistent with our findings for the aligned $\mathrm{H}_{2}{ }^{+}$(see Section 3.1).

We note that at the longer time ( $80 \mathrm{fs}$ ) the dissociative contributions to the CENFs are delocalized over the shell from $5 \mathrm{a}_{0}$ to $20 \mathrm{a}_{0}$, while the vibrational contributions remain confined to a sphere of radius $4 \mathrm{a}_{0}$. The interference contribution (also confined to a sphere of radius $4 \mathrm{a}_{0}$ ) is essentially ignorable (compare the magnitude of the interference component at $8 \mathrm{fs}$ and $80 \mathrm{fs}$ ), which suggests that the vibrational and dissociative contributions are no longer overlapping (i.e., the pulsating and exploding bubbles are well separated). Hence, at $80 \mathrm{fs}$, the dissociative part already represents two protons traveling in opposite directions, each carrying the electron with 0.5 probability. This analysis suggests that the flux density is a valuable tool for separating the vibrational and dissociative components. For example, one can judge either whether these components overlap, or, in an equivalent way, whether the interference is sufficiently small to indicate a proper separation of the components. This analysis is also consistent with the "virtual detector" method, ${ }^{88}$ which computes the nuclear momentum distribution from the NFD. 
$8 \mathrm{fs}$
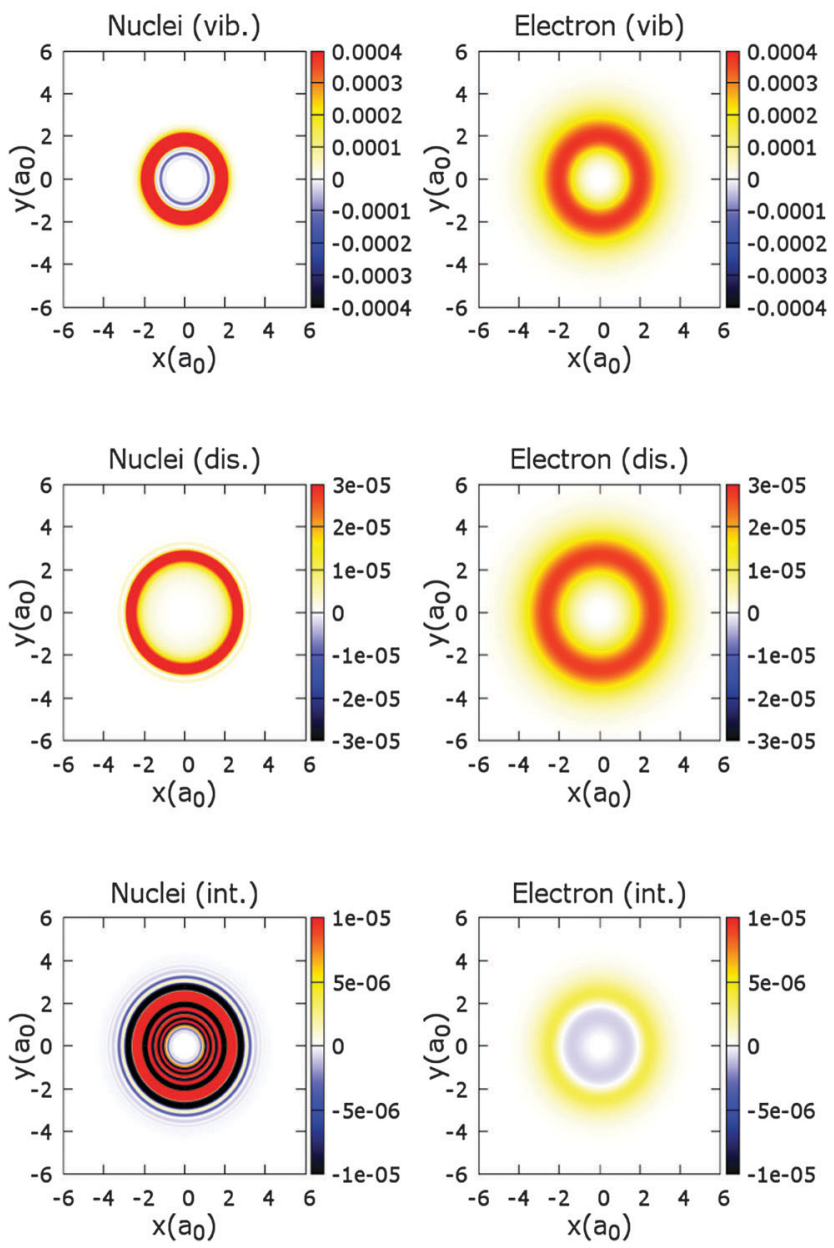

$80 \mathrm{fs}$
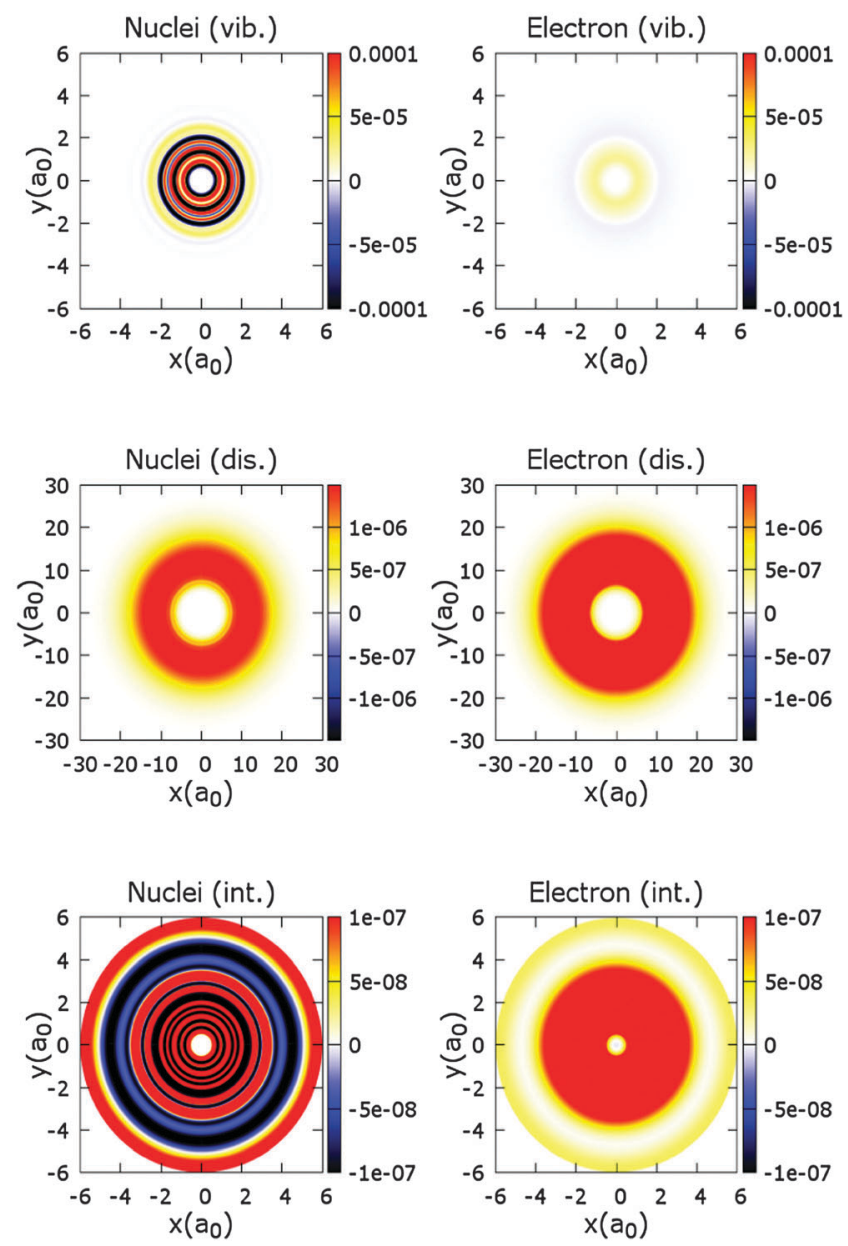

Fig. 7 Contour plots in the $x-y$ plane of vibrational (vib) dissociative (dis) and interfering (int) contributions to radial nuclear flux density of nucleus a (NFD) and electronic flux density (EFD) with respect to NCM for "quantum bubble" (isotropic $\mathrm{H}_{2}{ }^{+}$in electronic and rotational ground state ${ }^{2} \Sigma_{\mathrm{g}}{ }^{+}(\mathrm{J}=0$, $M=0))$. To improve visibility of dissociative component at large distance $\operatorname{EFD}\left(j_{\mathrm{e}}(r, t)\right)$ and $\operatorname{NFD}\left(j_{a}(R, t)\right)$ are multiplied by $4 \pi r^{2}$ and $4 \pi R^{2}$, respectively. The units are $E_{\mathrm{h}} \hbar^{-1}$.

\section{Concerted electronic and nuclear fluxes in tunnelling isomerization of $B_{4}$}

This section serves two purposes: (1) to present the first quantum results on CENFs that accompany the isomerization of a small inorganic molecule in the electronic ground state; (2) to exemplify the general quantum-theoretical approach (Section 2) applied to systems more demanding than the prototype (see Section 3). Tunnelling isomerization, first investigated by Hund in $1927,{ }^{73}$ remains an important topic in experimental and theoretical reaction dynamics. ${ }^{89,90}$ Specifically, we consider tunnelling isomerization of the boron cluster $\mathrm{B}_{4}$ in the electronic ground state from its $D_{2 \mathrm{~h}}$ rhombic structure ${ }^{91-93}$ ("reactant", R) through the $D_{4 \mathrm{~h}}$ square transition state (TS) to another, equivalent $D_{2 \mathrm{~h}}$ rhombic structure ("product", P), as illustrated in Fig. 8.

The rhombic $\mathrm{B}_{4}$ unit (also the triangular $\mathrm{B}_{3}$ unit) is a key structural block for building boron clusters $\mathrm{B}_{n}{ }^{-/ 0 /+}$, which have been confirmed experimentally to be planar, quasi-planar, or tubular in a wide range of sizes up to $\mathrm{B}_{40}{ }^{-/ 0}, \mathrm{~B}_{41}{ }^{+}$, and $\mathrm{B}_{42}{ }^{2+}$, the

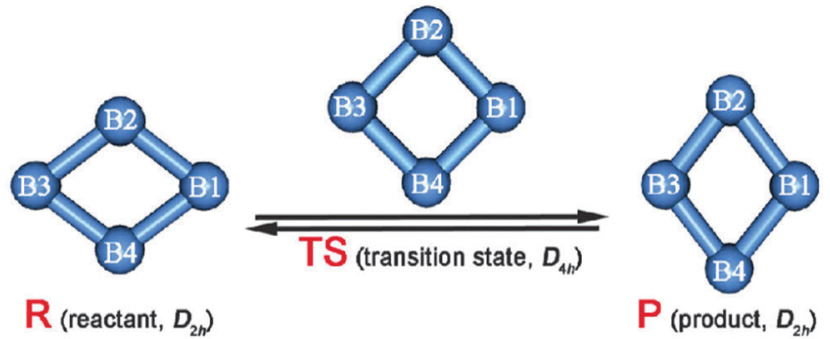

Fig. 8 Tunnelling isomerization of $\mathrm{B}_{4}$.

first cage-like all-boron fullerenes (or borospherenes). ${ }^{94-104}$ Doublechain boron ribbons ${ }^{105-107}$ and atomically thin $2 \mathrm{D}$ boron sheets and nanotubes ${ }^{108-111}$ also appear to consist of rhombic $\mathrm{B}_{4}$ building blocks. Owing to the electron deficiency of boron $\left[1 \mathrm{~s}^{2} 2 \mathrm{~s}^{2} 2 \mathrm{p}^{1}\right]$ compared to carbon $\left[1 \mathrm{~s}^{2} 2 \mathrm{~s}^{2} 2 \mathrm{p}^{2}\right],{ }^{105-107}$ the role of $\mathrm{B}_{4}$ in building larger $\mathrm{B}_{n}$ species is analogous to that of the $\mathrm{C}_{2}$ unit in building carbon fullerenes and hydrocarbons. 
Isomerizations of $\mathrm{B}_{4}$ fragments also contribute to the molecular rearrangements of the larger boron clusters. ${ }^{112}$

The importance of $\mathrm{B}_{4}$ calls for in-depth investigations of its properties. Its geometry, the coplanarity of $\mathrm{R}, \mathrm{P}$ and the transition state TS, the distortion of the square TS $\left(D_{4 \mathrm{~h}}\right)$ to the $D_{2 \mathrm{~h}}$ structures of $\mathrm{R}$ and $\mathrm{P}$ due to the pseudo-Jahn-Teller effect, the conservation of the $D_{2 \mathrm{~h}}$ symmetry in the transition from R to $\mathrm{P}$, as well as the nature of the chemical bonding in $\mathrm{B}_{4}$, which features both $\pi$ and $\sigma$ aromaticity, have already been elucidated. ${ }^{91-93}$ Moreover, since the energies of the first excited electronic triplet and singlet states at 1.1 and $3.1 \mathrm{eV}$, respectively, are much higher than the rather low value $V_{\mathrm{TS}}(\sim 0.031-$ $0.035 \mathrm{eV}$ ) at the $\mathrm{TS},{ }^{92,93}$ it is safe to assume that the tunnelling isomerization of $\mathrm{B}_{4}$ proceeds in the electronic ground state. The favorable properties of $\mathrm{B}_{4}$ make it an ideal candidate for discovering the first CENFs during tunnelling isomerization of a small inorganic molecule.

In the development of the theory in Section 2 we distinguish among several levels of approximation as "without BOA", "with BOA," and "beyond BOA". These are exemplified in the present treatment of tunnelling isomerization of $\mathrm{B}_{4}$. We now summarize the results of our study that are acquired through 11 steps detailed below. (1) We emphasize that synchronicity of the CENFs (see eqn (63) and (64)) is an analytical consequence of the Schrödinger equation (eqn (10) and (A.2)) "without the BOA." Complementary results "with BOA" include the following: (2) the structures of $\mathrm{R}$ and $\mathrm{P}$ at the minima of the symmetric double-well potential in the electronic ground state; (3) the symmetry-adapted set of main directions of the NFDs from R to $\mathrm{P}$; (4) the construction of the internal mobile-type coordinates $Q_{1}, \ldots, Q_{9}$ (see eqn (18)) for the nuclear motions; (5) the construction of a $1 \mathrm{D}$ model; (6) evaluations of the tunnelling splitting $\Delta E_{\mathrm{BOA}}$ and the related tunnelling time $\tau_{\mathrm{BOA}}=h / \Delta E_{\mathrm{BOA}}$; (7) computation of the NPDs of R and P, their difference and (8) the resulting NFDs in the main directions from $R$ to $P$; (9) computation of the EPDs of R and P and their difference; (10) determination of the symmetry-adapted set of the main directions of the EFDs. (11) In contrast with steps (2)-(10), the EFDs in the main directions from $\mathrm{R}$ to $\mathrm{P}$ are evaluated "beyond the BOA", because the BOA would yield zero EFDs (see eqn (71)). Steps (9)-(11) also involve an extension beyond the general theory of Section 2 and its application to the oneelectron prototype in Section 3, i.e., we adopt a common practice of quantum chemistry, which is valid for $\mathrm{B}_{4}$ and many other molecules, and express the EPD as a sum of contributions from electrons in core and valence orbitals. These are related to corresponding fluxes of core and valence electrons. $^{44}$

\section{Step 1}

From the outset all EPDs and NPDs during coherent tunnelling of $\mathrm{B}_{4}$ evolve as superpositions of the densities of $\mathrm{R}$ and $\mathrm{P}$ [see eqn (55)-(61)]. All of them are periodic (i.e., starting from $\mathrm{R}$ at $t=0$, arriving at $\mathrm{P}$ at $t=\tau / 2$, and returning to $\mathrm{R}$ at $t=\tau$, etc.) with the same period $\tau$. Likewise, all electronic and nuclear fluxes in any direction, in particular in the main directions, evolve periodically with the same period $\tau$. This means perfectly synchronous CENFs during coherent tunnelling isomerizations. All CENFs can be expressed as products of spatial factors and the same temporal factor, namely $(\pi / \tau) \sin (2 \pi t / \tau)$. Thus, for tunnelling isomerizations starting from $\mathrm{R}$ at $t=0$, all CENFs vanish at $t=0, \tau / 2, \tau$, etc. After pointing "forward" from $\mathrm{R}$ to $\mathrm{P}$ during the period $0 \leq t \leq \tau / 2$, all CENFs change sign and point "backward" from $\mathrm{P}$ to $\mathrm{R}$ during the period $\tau / 2 \leq t \leq \tau$, etc. Maximum amplitudes of all CENFs are observed halfway between $\mathrm{R}$ and $\mathrm{P}$ or (with opposite directions) halfway between $\mathrm{P}$ and $\mathrm{R}$, at the times $t=\tau / 4$ and $t=3 \tau / 4$, when $\sin (2 \pi t / \tau)=1$ or -1 , respectively.

\section{Step 2}

For the purpose of determining the structures of $\mathrm{R}$ and $\mathrm{P}$ at the two minima of the double-well potential, the electronic energy eigenvalue problem [see eqn (66a)] is solved by means of the coupled-cluster technique with single, double and perturbative triple excitations $(\operatorname{CCSD}(\mathrm{T}))^{113}$ combined with Dunning's augmented triple-zeta correlation-consistent basis set (aug-ccpVTZ). ${ }^{114}$ The corresponding canonical molecular orbitals (CMOs) are pictured in Fig. 9. It is convenient to set the values of the potential energy surface (PES) at the potential minima equal to zero. The result for $\mathrm{R}$ is the rhombic structure shown in Fig. 8. For convenience we assume that the NCM is at the origin of the laboratory-fixed coordinate system, and that the long and short molecular principal axes of $\mathrm{R}$ are aligned parallel with the laboratory $x$ - and $y$-axes (horizontal and vertical directions in Fig. 8), respectively (e.g., by means of the methods of ref. 60-65). Furthermore, we assume that the molecule does not

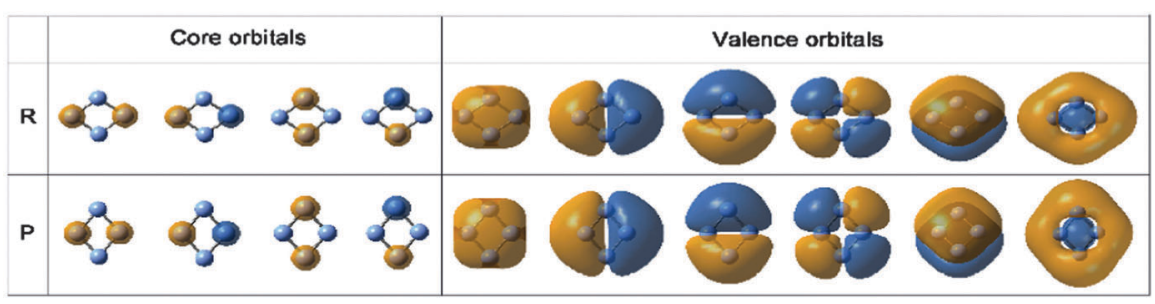

Fig. 9 Symmetry-adapted canonical molecular orbitals (CMOs) of $\mathrm{B}_{4}$. Yellow and blue colors correspond to positive and negative lobes, respectively. Top row: CMOs of rhombic reactant (R), in order of increasing orbital energies, from four core CMOs $(k=1,2,3,4)$ to six valence $C M O s(k=5,6,7,8$, $9,10)$. Nuclear configuration is fixed at potential minimum for R. Bottom row: CMOs of rhombic product $(P)$ with nuclear configuration fixed at potential minimum for $\mathrm{P}$, in order which correlates with CMOs of $\mathrm{R}$. 
rotate and that its $D_{2 \mathrm{~h}}$ symmetry is preserved in the transition from $\mathrm{R}$ to $\mathrm{P} .{ }^{91-93}$ This implies that the structures of $\mathrm{R}$ and $\mathrm{P}$ are equivalent and coplanar, but with interchanged alignments of the long and short axes (see Fig. 8). Accordingly, on the way from $\mathrm{R}$ to $\mathrm{P}$ the pair of nuclei labeled 1 (right) and 3 (left), as well as the pair labelled 2 (top) and 4 (bottom), move in opposite directions parallel with the $x$ - and $y$-axes, respectively. The nuclei of the 1-3 pair are equidistant from the NCM; the same is true of the $2-4$ pair.

We note in passing that conservation of $D_{2 \mathrm{~h}}$ symmetry ${ }^{91-93}$ implies conservation of the ring structures (see Fig. 8) (i.e., nonring geometries are not feasible during coherent tunnelling from $\mathrm{R}$ to $\mathrm{P}$ ). As a consequence, the relevant molecular symmetry group is a very small subgroup of the complete nuclear permutation inversion group, namely $\mathrm{G}\left(\mathrm{B}_{4}\right.$, tunnelling) = $\left\{E,(14)(23)^{*}\right\}$, which is isomorphic with $C_{\mathrm{s}}(M)$. The eigenfunctions $\psi_{0}$ and $\psi_{1}$ of the lowest tunnelling doublet transform according to its irreducible representations $\mathrm{A}^{\prime}$ and $\mathrm{A}^{\prime \prime}$, respectively.

\section{Step 3}

The symmetry-adapted set of the main directions of the nuclear fluxes from $\mathrm{R}$ to $\mathrm{P}$ are illustrated in Fig. 10a by arrows pointing from the positions of the nuclei $1,2,3$ and 4 in $\mathrm{R}$ to their positions in P. Fig. 10a also depicts the difference between the NPDs of P and R (see Section 2).

\section{Step 4}

The $3 N_{\mathrm{n}}=12$ nuclear coordinates, which consist of nine internal nuclear mobile-type coordinates $Q_{1}, Q_{2}, \ldots, Q_{9}$ in the NCM frame, supplemented by the three coordinates $S_{x}, S_{y}, S_{z}$ of the NCM (see eqn (18)), are determined as follows. The general theory of Section 2 suggests that the first coordinate $Q_{1}$ corresponds to the main direction of the fluxes of the nuclei during coherent tunnelling isomerization from $\mathrm{R}$ to $\mathrm{P}$. According to the results of steps 2 and 3 (summarized in Fig. 8 and 10a), the nuclei of the 1-3 pair and the 2-4 pair move in opposition. Hence we define the internal nuclear coordinate as

$$
Q_{1}=-X_{1}+Y_{2}+X_{3}-Y_{4}=\left(Y_{2}-Y_{4}\right)-\left(X_{1}-X_{3}\right)
$$

Note that by definition $Q_{1}$ is symmetry-adapted (i.e., motion along $Q_{1}$ conserves the rhombic shape of $\left.\mathrm{B}_{4}\left(D_{2 \mathrm{~h}}\right)\right)$. The second equality of eqn (98) shows that $Q_{1}$ is the difference between the $y$-coordinates of nuclei 2 and 4 and the $x$-coordinates of nuclei 1 and 3. The values $Q_{1 \mathrm{R}}$ and $Q_{1 \mathrm{P}}$ for the $\mathrm{R}$ and $\mathrm{P}$ configurations are negative and positive, respectively, with the same absolute values (i.e., $\left|Q_{1 \mathrm{R}}\right|=Q_{1 \mathrm{P}}$ ). Halfway between $\mathrm{R}$ and $\mathrm{P}$, the value of $Q_{1}$ is $Q_{1 \mathrm{~b}}=\left(Q_{1 \mathrm{R}}+Q_{1 \mathrm{P}}\right) / 2=0$, corresponding to square geometry of $\mathrm{B}_{4}$ at the potential barrier. Using the diagonal version of the general expression for the reduced mass (given just below eqn (17)), we obtain the reduced mass associated with $Q_{1}: 1 / \mu_{1}=\left(1 / M_{\mathrm{B}}\right)\left[(-1)^{2}+1^{2}+1^{2}+(-1)^{2}\right]=4 / M_{\mathrm{B}}$.

The construction of $Q_{1}$ as a mobile-type coordinate is also apparent in eqn (98). Thus, the pair of nuclei 1 and 3 and the pair 2 and 4 may be regarded as diatomic subgroups oriented perpendicularly to each other, with corresponding internuclear distances $\left(X_{1}-X_{3}\right)$ and $\left(Y_{2}-Y_{4}\right)$, respectively, and with reduced masses $\mu=M_{\mathrm{B}} M_{\mathrm{B}} /\left(M_{\mathrm{B}}+M_{\mathrm{B}}\right)=M_{\mathrm{B}} / 2$. The mobile-type coordinate $Q_{1}$ (eqn (98)) may then be interpreted as the "distance" from one quasi-particle (the 1-3 pair) with reduced mass $\mu$ and "coordinate" $\left(X_{1}-X_{3}\right)$ to the other quasi-particle (the 2-4 pair) with reduced mass $\mu$ and "coordinate" $\left(Y_{2}-Y_{4}\right)$. The reduced mass of the subsystem comprising the diatomic subgroups is then $\mu_{1}=\mu \cdot \mu /(\mu+\mu)=\mu / 2=M_{\mathrm{B}} / 4$.

Another way to rationalize the reduced mass associated with $Q_{1}$ is based on an Ehrenfest-type picture, according to which the kinetic energy of motion along $Q_{1}$ with associated mass $\mu_{1}$ from $\mathrm{R}$ to $\mathrm{P}$ should account for the separate kinetic energies of the boron atoms, all having mass $M_{\mathrm{B}}$. That is, we should require that $\mu_{1}\left\langle\dot{Q}_{1}\right\rangle^{2} / 2=M_{\mathrm{B}}\left(\left\langle\dot{X}_{1}\right\rangle^{2}+\left\langle\dot{Y}_{2}\right\rangle^{2}+\left\langle\dot{X}_{3}\right\rangle^{2}+\left\langle\dot{Y}_{4}\right\rangle^{2}\right) / 2$. The synchronicity of the nuclear motions implies equivalent time evolutions of the form $\left\langle Q_{1}(t)\right\rangle=\left\langle Q_{1}\right\rangle_{\mathrm{R}}+\left\langle\Delta Q_{1}\right\rangle \lambda(t),\left\langle X_{i}(t)\right\rangle=\left\langle X_{i}\right\rangle_{\mathrm{R}}+\left\langle\Delta X_{i}\right\rangle \lambda(t)$,

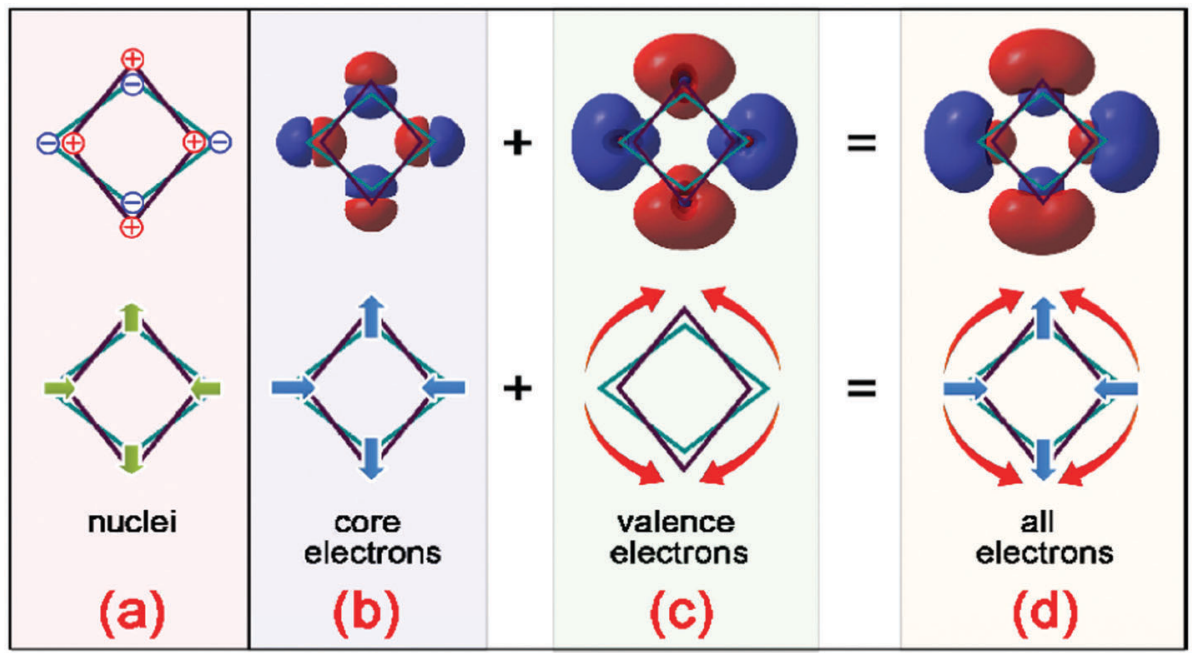

Fig. 10 Differences $\left\langle\Delta \rho_{\mathrm{e}, \mathrm{NCM}}(\mathbf{x})\right\rangle_{\mathrm{BOA}}$ (eqn (117c))) and $\left\langle\Delta \rho_{1}\left(Q_{1}\right)\right\rangle_{\mathrm{BOA}}$ (eqn (103c) of electronic [(b)-(d)] and nuclear (a) probability densities of product and reactant (top), and corresponding main directions of concerted electronic and nuclear fluxes during tunnelling isomerization of $B_{4}$ (bottom). 


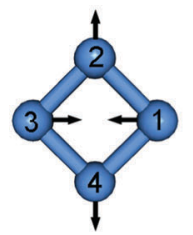

$Q_{1}$

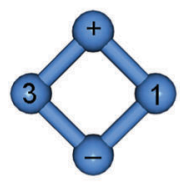

$Q_{7}$

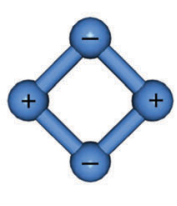

$Q_{2}$

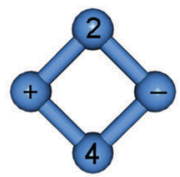

$\mathrm{Q}_{8}$

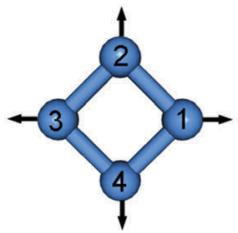

$Q_{3}$

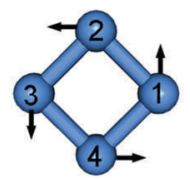

Q9

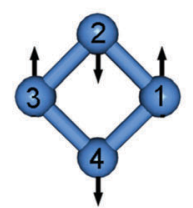

$Q_{4}$

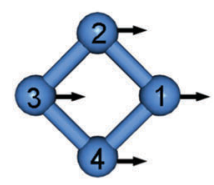

$\mathrm{S}_{\mathrm{x}}$
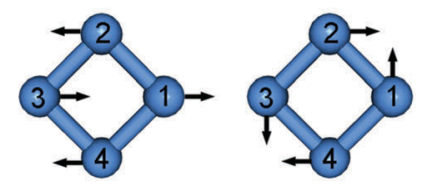

$Q_{5}$
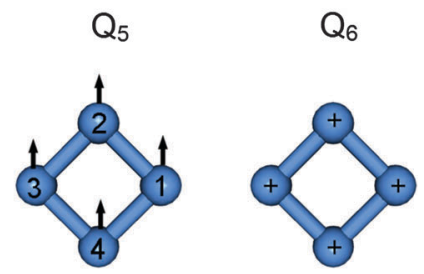

$\mathrm{S}_{\mathrm{y}}$

$\mathrm{S}_{z}$

Fig. 11 Nine symmetry-adapted coordinates $Q_{1}, \ldots, Q_{9}$ for internal nuclear motions of $B_{4}$, together with coordinates $S_{x}, S_{y}, S_{z}$ for nuclear center of mass of $\mathrm{B}_{4}$. Arrows indicate direction of motion of individual nuclei; + and - signs indicate motion either out of, or into, plane of page, respectively.

and $\left\langle Y_{i}(t)\right\rangle=\left\langle Y_{i}\right\rangle_{\mathrm{R}}+\left\langle\Delta Y_{i}\right\rangle \lambda(t)$, where $\left\langle\Delta Q_{1}\right\rangle,\left\langle\Delta X_{i}\right\rangle$ and $\left\langle\Delta Y_{i}\right\rangle$ stand for the mean values of the distances of $Q_{1}, X_{i}$, and $Y_{i}$ from R to $\mathrm{P}$ and $\lambda(t)=\sin ^{2}(\pi t / \tau)$ (see eqn (59a)). Hence, we have

$$
\mu_{1}\left\langle\Delta Q_{1}\right\rangle^{2}=M_{\mathrm{B}}\left(\left\langle\Delta X_{1}\right\rangle^{2}+\left\langle\Delta Y_{2}\right\rangle^{2}+\left\langle\Delta X_{3}\right\rangle^{2}+\left\langle\Delta Y_{4}\right\rangle^{2}\right)
$$

The symmetry of $\mathrm{B}_{4}$ implies that $-\left\langle\Delta X_{1}\right\rangle=\left\langle\Delta Y_{2}\right\rangle=\left\langle\Delta X_{3}\right\rangle=$ $-\left\langle\Delta Y_{4}\right\rangle=\langle\Delta\rangle$. Thus, eqn (98) yields $\left\langle\Delta Q_{1}\right\rangle=4\langle\Delta\rangle$. Using eqn (99a), we obtain $\mu_{1}\langle 4 \Delta\rangle^{2}=4 M_{\mathrm{B}}\langle\Delta\rangle^{2}$ and consequently $\mu_{1}=M_{\mathrm{B}} / 4$. We note that eqn (99a) is well approximated by the classical limit

$$
\mu_{1} \Delta Q_{1}^{2}=M_{\mathrm{B}}\left(\Delta X_{1}^{2}+\Delta Y_{2}^{2}+\Delta X_{3}^{2}+\Delta Y_{4}^{2}\right)
$$

where

$\Delta Q_{1}=-\Delta X_{1}+\Delta Y_{2}+\Delta X_{3}-\Delta Y_{4}=\left|\Delta R_{1}\right|+\left|\Delta R_{2}\right|+\left|\Delta R_{3}\right|+\left|\Delta R_{4}\right|$
The reduced mass $\mu_{1}$ associated with $Q_{1}$ is thus rather small. Below we employ the isotope ${ }^{11} \mathrm{~B}$, which has mass $M_{\mathrm{B}}=$ $11.009 \mathrm{u}$. Hence, $\mu_{1}=2.752 \mathrm{u}$. The small reduced mass, together with the low potential barrier, yields an extremely short tunnelling time (see eqn (52b) and step 6 below). Incidentally, the coordinate $Q_{1}$ is equivalent to a normal coordinate of $\mathrm{B}_{4}$, namely the antisymmetric stretch.

There are many ways to construct the remaining mobile-type coordinates $Q_{2}, \ldots, Q_{9}$. We choose $Q_{2}, \ldots, Q_{6}$ and $Q_{7}, \ldots, Q_{9}$ to correspond with the other normal vibrational modes, and with rotations, respectively. The mobile-type coordinates, $Q_{1}, \ldots, Q_{9}$, together with the coordinates $S_{x}, S_{y}, S_{z}$ of the NCM, are defined explicitly by

$$
\left(\begin{array}{l}
Q_{1} \\
Q_{2} \\
Q_{3} \\
Q_{4} \\
Q_{5} \\
Q_{6} \\
Q_{7} \\
Q_{8} \\
Q_{9} \\
S_{x} \\
S_{y} \\
S_{z}
\end{array}\right)=\left(\begin{array}{cccccccccccc}
-1 & 0 & 0 & 0 & 1 & 0 & 1 & 0 & 0 & 0 & -1 & 0 \\
0 & 0 & 1 / 2 & 0 & 0 & -1 / 2 & 0 & 0 & 1 / 2 & 0 & 0 & -1 / 2 \\
1 / 2 & 0 & 0 & 0 & 1 / 2 & 0 & -1 / 2 & 0 & 0 & 0 & -1 / 2 & 0 \\
0 & 1 / 2 & 0 & 0 & -1 / 2 & 0 & 0 & 1 / 2 & 0 & 0 & -1 / 2 & 0 \\
1 / 2 & 0 & 0 & -1 / 2 & 0 & 0 & 1 / 2 & 0 & 0 & -1 / 2 & 0 & 0 \\
0 & 1 & 0 & 1 & 0 & 0 & 0 & -1 & 0 & -1 & 0 & 0 \\
0 & 0 & 0 & 0 & 0 & 1 & 0 & 0 & 0 & 0 & 0 & -1 \\
0 & 0 & -1 & 0 & 0 & 0 & 0 & 0 & 1 & 0 & 0 & 0 \\
0 & 1 / 2 & 0 & -1 / 2 & 0 & 0 & 0 & -1 / 2 & 0 & 1 / 2 & 0 & 0 \\
1 / 4 & 0 & 0 & 1 / 4 & 0 & 0 & 1 / 4 & 0 & 0 & 1 / 4 & 0 & 0 \\
0 & 1 / 4 & 0 & 0 & 1 / 4 & 0 & 0 & 1 / 4 & 0 & 0 & 1 / 4 & 0 \\
0 & 0 & 1 / 4 & 0 & 0 & 1 / 4 & 0 & 0 & 1 / 4 & 0 & 0 & 1 / 4
\end{array}\right)\left(\begin{array}{c}
X_{1} \\
Y_{1} \\
Z_{1} \\
X_{2} \\
Y_{2} \\
Z_{2} \\
X_{3} \\
Y_{3} \\
Z_{3} \\
X_{4} \\
Y_{4} \\
Z_{4}
\end{array}\right)
$$


or in compact matrix notation $\tilde{\mathbf{Q}}=\mathbf{W R}$ [see eqn (18)]. The movements of individual nuclei associated with the modes $Q_{1}$, $Q_{2}, \ldots, S_{z}$ are illustrated in Fig. 11. The coordinate $Q_{2}$ describes the out-of-plane bending mode ("butterfly"), $Q_{3}$ the symmetric stretch, and so on. Likewise, $Q_{7}, Q_{8}$, and $Q_{9}$ correlate with rotations about the $x$-, $y$ - and $z$-axes, respectively, and $S_{x}, S_{y}$, and $S_{z}$ describe translations of the NCM in the $x$-, $y$-, and $z$-directions, respectively. Hence the PES should depend on $Q_{1}, \ldots, Q_{6}$, but not on $Q_{7}, Q_{8}$, and $Q_{9}$ or $S_{x}, S_{y}$, and $S_{z}$.

Using eqn (20) and the masses $M_{1}=M_{2}=M_{3}=M_{4}=M_{\mathrm{B}}$, we can write the kinetic energy operator as

$$
\begin{aligned}
T_{\mathrm{n}}= & P_{1}^{2} / 2\left(M_{\mathrm{B}} / 4\right)+P_{2}{ }^{2} / 2 M_{\mathrm{B}}+P_{3}{ }^{2} / 2 M_{\mathrm{B}}+P_{4}{ }^{2} / 2 M_{\mathrm{B}}+P_{5}{ }^{2} / 2 M_{\mathrm{B}} \\
& +P_{6}{ }^{2} / 2\left(M_{\mathrm{B}} / 4\right)+P_{7}{ }^{2} / 2 M_{\mathrm{B}}+P_{8}{ }^{2} / 2 M_{\mathrm{B}}+P_{9}{ }^{2} / 2 M_{\mathrm{B}}+P_{10}{ }^{2} / 2\left(4 M_{\mathrm{B}}\right) \\
& +P_{11}{ }^{2} / 2\left(4 M_{\mathrm{B}}\right)+P_{12}{ }^{2} / 2\left(4 M_{\mathrm{B}}\right)
\end{aligned}
$$

The procedure is detailed in Appendix B. The diagonal form of $T_{\mathrm{n}}$ confirms the definitions of the coordinates $Q_{1}, \ldots, Q_{9}$ as mobile-type coordinates. The associated reduced masses appear in the denominators in eqn (101). In particular, that $\mu_{1}=M_{\mathrm{B}} / 4$ validates the construction of $Q_{1}$ described above.

The rotational coordinates $Q_{7}, Q_{8}$, and $Q_{9}$ are set to zero (i.e., these "frozen rotations" correspond to the present model of the oriented $\mathrm{B}_{4}$, as illustrated in Fig. 8). Likewise, the NCM coordinates $S_{x}, S_{y}$, and $S_{z}$ are set to zero. The two minima of the PES corresponding to $\mathrm{R}$ and $\mathrm{P}$ are then located at

$$
Q_{1 \mathrm{R}}=-0.53 \AA, Q_{2 \mathrm{R}}=0, Q_{3 \mathrm{R}}=8.68 \AA \text {, }
$$

$$
\begin{gathered}
Q_{4 \mathrm{R}}=Q_{5 \mathrm{R}}=Q_{6 \mathrm{R}}=0, \\
Q_{1 \mathrm{P}}=-Q_{1 \mathrm{R}}, Q_{2 \mathrm{P}}=Q_{2 \mathrm{R}}, Q_{3 \mathrm{P}}=Q_{3 \mathrm{R}}, \\
Q_{4 \mathrm{P}}=Q_{4 \mathrm{R}}, Q_{5 \mathrm{P}}=Q_{5 \mathrm{R}}, Q_{6 \mathrm{P}}=Q_{6 \mathrm{R}}
\end{gathered}
$$

The Cartesian coordinates related to $Q_{1}$ under the constraints of eqn (102) are given by $\mathbf{R}_{\mathrm{R}}=\tilde{\mathbf{W}}^{-1} \mathbf{Q}_{\mathrm{R}}$ and $\mathbf{R}_{\mathrm{P}}=\tilde{\mathbf{W}}^{-1} \mathbf{Q}_{\mathrm{P}}$, according to eqn (18). The corresponding structures of $\mathrm{R}$ and $\mathrm{P}$ are pictured in Fig. 8.

\section{Step 5}

For the purpose of reducing the full 12D model to the 1D model described in terms of only $Q_{1}$, we impose the following 11 $(=12-1)$ constraints: the NCM is fixed at the origin, i.e., $S_{x}=$ $S_{y}=S_{z}=0$; the molecular orientations are also fixed at $Q_{7}=Q_{8}=Q_{9}=0$; conservation of $D_{2 \mathrm{~h}}$ symmetry implies $Q_{2}=Q_{4}=Q_{5}=Q_{6}=0 ; Q_{3}=Q_{3 \mathrm{R}}=Q_{3 \mathrm{P}}=8.68 \AA$. This last constraint corresponds to replacing the intrinsic reaction coordinate (IRC) by a straight line. The IRC embedded in the 2D section $V\left(Q_{1}, Q_{2}=0, Q_{3}, Q_{4}=Q_{5}=Q_{6}=0\right)$ of the PES is shown in Fig. 12a. The IRC is evidently almost linear (i.e., the constraint $Q_{3}=Q_{3 \mathrm{R}}=$ $Q_{3 \mathrm{P}}$ is an excellent approximation). Henceforward we refer to the resulting $1 \mathrm{D}$ cut of the PES, $V\left(Q_{1}, Q_{2}=0, Q_{3}=Q_{3 \mathrm{R}}=Q_{3 \mathrm{P}}, Q_{4}=Q_{5}=\right.$ $\left.Q_{6}=0\right)$, illustrated in Fig. 12b, simply as $V\left(Q_{1}\right)$.

Thus we arrive at the $1 \mathrm{D}$ model having the Hamiltonian $H=T_{\mathrm{n}}+H_{\mathrm{e}}$ and Schrödinger equation specified in eqn (26) and (27). With the internal and NCM coordinates $Q_{2}, \ldots, S_{z}$ fixed

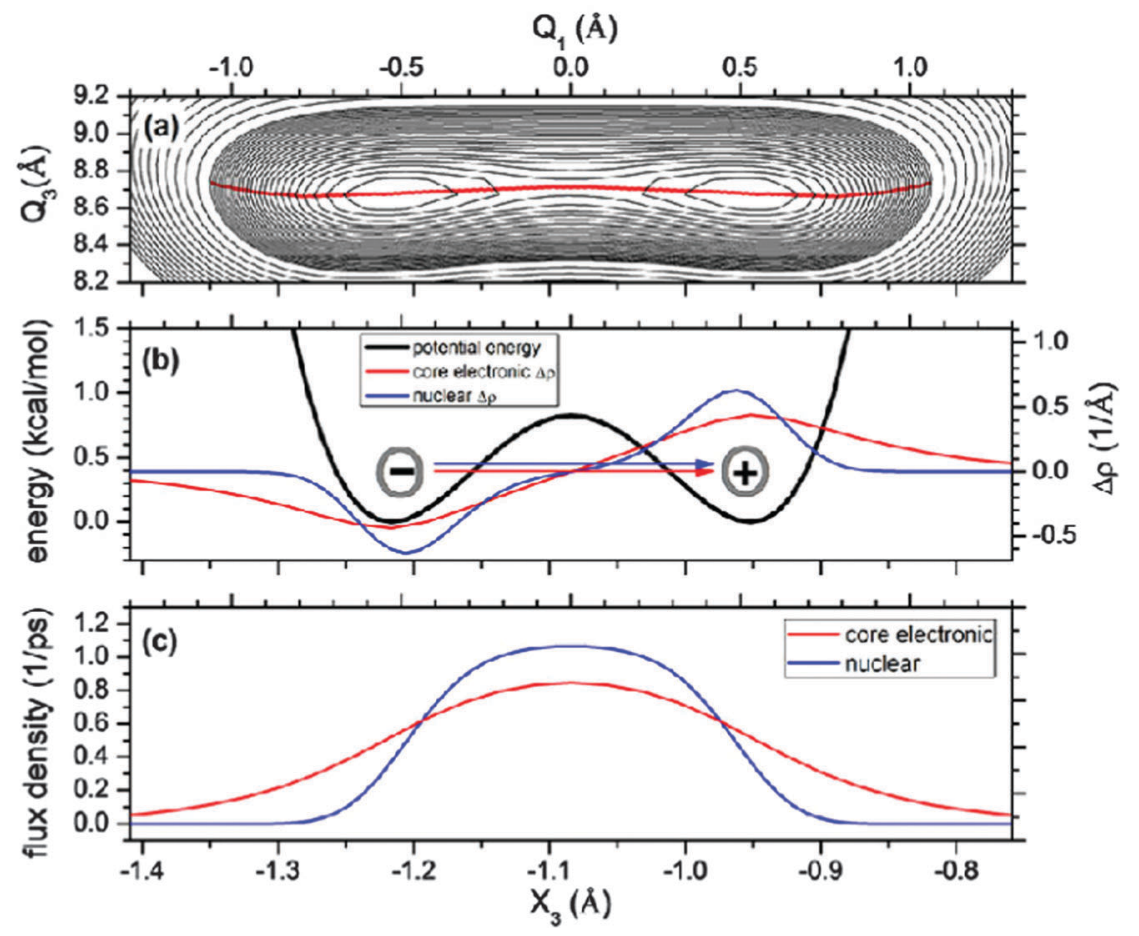

Fig. 12 (a) Two-dimensional (2D) contour plot of PES, showing intrinsic reaction coordinate (IRC) of $\mathrm{B}_{4}$ in red. Coordinates $Q_{1}$ and $Q_{3}$ are illustrated in Fig. 11. (b) Potential curve for $1 D$ model, plotted versus main direction $Q_{1}$ (or, exemplarily, versus Cartesian coordinate $X_{3}$ of nucleus 3 ). Also shown are differences between probability densities of product $P$ and reactant $R$ versus $Q_{1}$, or equivalently, for nucleus 3 (blue) and for an electron in $1 \mathrm{~s}$ core orbital (red) versus $X_{3}$. Superimposed arrows in (b) indicate same directions of concerted fluxes (CENFs) from negative domains of density differences to positive ones. (c) Nuclear flux (blue) and flux of an electron in associated 1s core orbital (red) for nucleus 3. 
as indicated above, eqn (25) yields the Cartesian coordinates of all nuclei in the NCM frame as a function of $Q_{1}$. These nuclear motions are indicated by the arrows in Fig. 10a.

In the $1 \mathrm{D}$ model, the momentum operator $P_{1}=-i \hbar \partial / \partial Q_{1}$ is taken into account explicitly, whereas all other momenta are disregarded (i.e., $P_{2}=P_{3}=\ldots P_{12}=0$ ). Under these constraints eqn (20) relates the Cartesian components of the nuclear momenta in the NCM frame to the internal momentum $P_{1}$ and eqn (51) expresses the Cartesian components of the fluxes of nuclei observed in the NCM frame in terms of the internal flux $\left\langle j_{1}\right\rangle$.

\section{Step 6}

If the physics underlying the present $1 \mathrm{D}$ model is adequate, it should account for the essential properties of the tunnelling isomerization of $\mathrm{B}_{4}$. As a test of the model, we compare the tunnelling splitting of $\mathrm{B}_{4}$ of the full 12D model with that of the reduced $1 \mathrm{D}$ model. This test is motivated by the validation of the 1D model for tunnelling isomerization of another four-atom molecule, namely ammonia, under analogous constraints: fixed NCM, fixed orientation, conservation of symmetry, and approximate conservation of one specific internal nuclear coordinate (the mean $\mathrm{NH}$ bond length in $C_{3 \mathrm{v}}$ symmetry). The validation of the 1D model is based on excellent agreement of the computed tunnelling splittings with the experimental (i.e., inherently the full D) ones. ${ }^{115}$

To calculate the tunnelling splitting $\Delta E_{\mathrm{BOA}}$ and the related tunnelling time $\tau_{\mathrm{BOA}}=h / \Delta E_{\mathrm{BOA}}$ for $\mathrm{B}_{4}$, we take the $6 \mathrm{D}$ (corresponding to full $12 \mathrm{D}$ with fixed NCM and orientation) quantummechanical result for the tunnelling splitting $\Delta E_{\mathrm{BOA}}=21.9 \mathrm{hc} \mathrm{cm}^{-1}$, which has been reported in ref. 93 , as a reference. The calculations of ref. 93 are based on the qualitatively correct 6D PES, which is a polynomial fit (to fourth order) of $643 a b$ initio energies obtained at the $\operatorname{CCSD}(\mathrm{T}) / \mathrm{cc}-\mathrm{pVQZ}$ level of quantum chemistry. Using the same polynomial PES as ref. 93, we performed 1D calculations by setting all coordinates except $Q_{1}$ to the values at $\mathrm{R}$ and $\mathrm{P}$ (see eqn (102)). The resulting 1D tunnelling splitting $\Delta E_{\mathrm{BOA}}=21.7 \mathrm{hc} \mathrm{cm}^{-1}$ is in excellent agreement with the 6D reference. This validates the $1 \mathrm{D}$ model. Next, we replace the polynomial fit of ref. 93 by the $1 \mathrm{D}$ potential curve $V\left(Q_{1}\right)$ calculated at the same level of quantum chemistry. The resulting tunnelling splitting is $\Delta E_{\mathrm{BOA}}=13.5 \mathrm{hc} \mathrm{cm}^{-1}$. Alternatively, if we use $V\left(Q_{1}\right)$ evaluated at the present level of quantum chemistry $\left(\operatorname{CCSD}(\mathrm{T}) /(\right.$ aug-cc-pVTZ) $)$, then we get $\Delta E_{\mathrm{BOA}}=11.5 \mathrm{hc} \mathrm{cm}^{-1}$. Comparisons of the 6D and 1D results based on the polynomial fit to the PES, and the 1D results based on the potential curves $V\left(Q_{1}\right)$ at different levels of quantum chemistry, show that in the case of $\mathrm{B}_{4}$ the tunnelling splittings are far less sensitive to approximations (i.e., reduced dimensionality or level of quantum chemistry) compared with low-(fourth-) order polynomial fits to the PES. Below we use the value $\Delta E_{\mathrm{BOA}}=11.5 \mathrm{hc} \mathrm{cm}^{-1}$, corresponding to the tunnelling time $\tau_{\mathrm{BOA}}=2.9 \mathrm{ps}$. That this value is between those for ammonia (42 ps) ${ }^{115,116}$ and malonaldehyde $(1.5 \mathrm{ps})^{117}$ suggests that the predicted tunnelling time of $\mathrm{B}_{4}$ could be confirmed experimentally by means of analogous measurements, or by the techniques developed in ref. 118-120. The dependence of the tunnelling times on the levels of the quantum chemistry and on the dimensionality of the model will be reported elsewhere. In the subsequent expressions here, we use the notation $\tau$, dropping the subscript "BOA" to simplify the notation.

\section{Step 7}

To evaluate the NPDs of R and P, and their difference in the $\mathrm{BOA}$, we use the general expressions given by eqn (56c) and (57b), from which we derive

$$
\begin{gathered}
\left\langle\rho_{1}\left(Q_{1}\right)\right\rangle_{\mathrm{BOA}, \mathrm{R}}=\left|\chi_{\mathrm{R}}\left(Q_{1}\right)\right|^{2} \\
\left\langle\rho_{1}\left(Q_{1}\right)\right\rangle_{\mathrm{BOA}, \mathrm{P}}=\left|\chi_{\mathrm{P}}\left(Q_{1}\right)\right|^{2} \\
\left\langle\Delta \rho_{1}\left(Q_{1}\right)\right\rangle_{\mathrm{BOA}}=\left\langle\rho_{1}\left(Q_{1}\right)\right\rangle_{\mathrm{BOA}, \mathrm{P}}-\left\langle\rho_{1}\left(Q_{1}\right)\right\rangle_{\mathrm{BOA}, \mathrm{R}}
\end{gathered}
$$

where

$$
\begin{gathered}
\chi_{\mathrm{R}}\left(Q_{1}\right)=N\left[\chi_{0}\left(Q_{1}\right)+\chi_{1}\left(Q_{1}\right)\right] \\
\chi_{\mathrm{R}}\left(Q_{1}\right)=N\left[-\chi_{0}\left(Q_{1}\right)+\chi_{1}\left(Q_{1}\right)\right]
\end{gathered}
$$

are the nuclear wavefunctions representing $\mathrm{R}$ and $\mathrm{P}$ in terms of the eigenfunctions $\chi_{0}\left(Q_{1}\right)$ and $\chi_{1}\left(Q_{1}\right)$ of the lowest tunnelling doublet. The normalization constant is $N=1 / \sqrt{2}$. The 1D NPD difference $\left\langle\Delta \rho_{1}\left(Q_{1}\right)\right\rangle_{\mathrm{BOA}}$ is shown in Fig. 12b together with the arrows indicating the main directions of the nuclear flux from negative to positive domains of $\left\langle\Delta \rho_{1}\left(Q_{1}\right)\right\rangle_{\mathrm{BOA}}$. The time evolution of the NPD, given by the BOA analogue of the exact formula (see eqn (61a)),

$$
\left\langle\rho_{1}\left(Q_{1}, t\right)\right\rangle_{\mathrm{BOA}, \mathrm{R}}=\left\langle\rho_{1}\left(Q_{1}\right)\right\rangle_{\mathrm{BOA}, \mathrm{R}}+\left\langle\Delta \rho_{1}\left(Q_{1}\right)\right\rangle_{\mathrm{BOA}} \sin ^{2}(\pi t / \tau)
$$

is shown as a contour plot in Fig. 13. The transformation from $Q_{1}$ to the Cartesian coordinates $X_{1}, Y_{2}, X_{3}$, and $Y_{4}$ of the nuclei 1, 2, 3, and 4 (see eqn (25)) yields the corresponding time evolutions of the probability densities of the individual nuclei during tunnelling. This is also shown in Fig. 13 exemplarily for the tunnelling of nucleus 3 from $\mathrm{R}$ to $\mathrm{P}$.

\section{Step 8}

We determine the nuclear flux using the result of step 7 in the BOA expression,

$$
\left\langle j_{1}\left(Q_{1}, t\right)\right\rangle_{\mathrm{BOA}, \mathrm{R}}=-\int_{Q_{10}}^{Q_{1}} \mathrm{~d} Q_{1}{ }^{\prime}\left\langle\Delta \rho\left(Q_{1}{ }^{\prime}\right)\right\rangle_{\mathrm{BOA}}(\pi / \tau) \sin (2 \pi t / \tau)
$$

which is analogous to the exact formula given by eqn (64a). Robust results are obtained for a conservative choice of the lower limit of integration $Q_{10}(=-1.7 \AA)$. The time evolution of the resulting nuclear flux observed at $Q_{1}$ is also shown in Fig. 13, together with the corresponding nuclear flux of (exemplarily) boron nucleus B3 at $X_{3}$. As expected, the fluxes are directed from $\mathrm{R}$ to $\mathrm{P}$ during the period $0 \leq t \leq \tau / 2$, and then from $\mathrm{P}$ to $\mathrm{R}$ during the period $\tau / 2 \leq t \leq \tau$. The maximum amplitudes of $\left\langle j_{1}\left(Q_{1}, t=\tau / 4\right)\right\rangle_{\mathrm{BOA}, \mathrm{R}}$ are plotted in Fig. 12c.The nuclear flux evidently achieves its maximum value at the potential barrier. On first glance this is counterintuitive because the NPDs at the barrier are exceedingly small. The result may be rationalized as follows. ${ }^{20}$ The nuclei do 

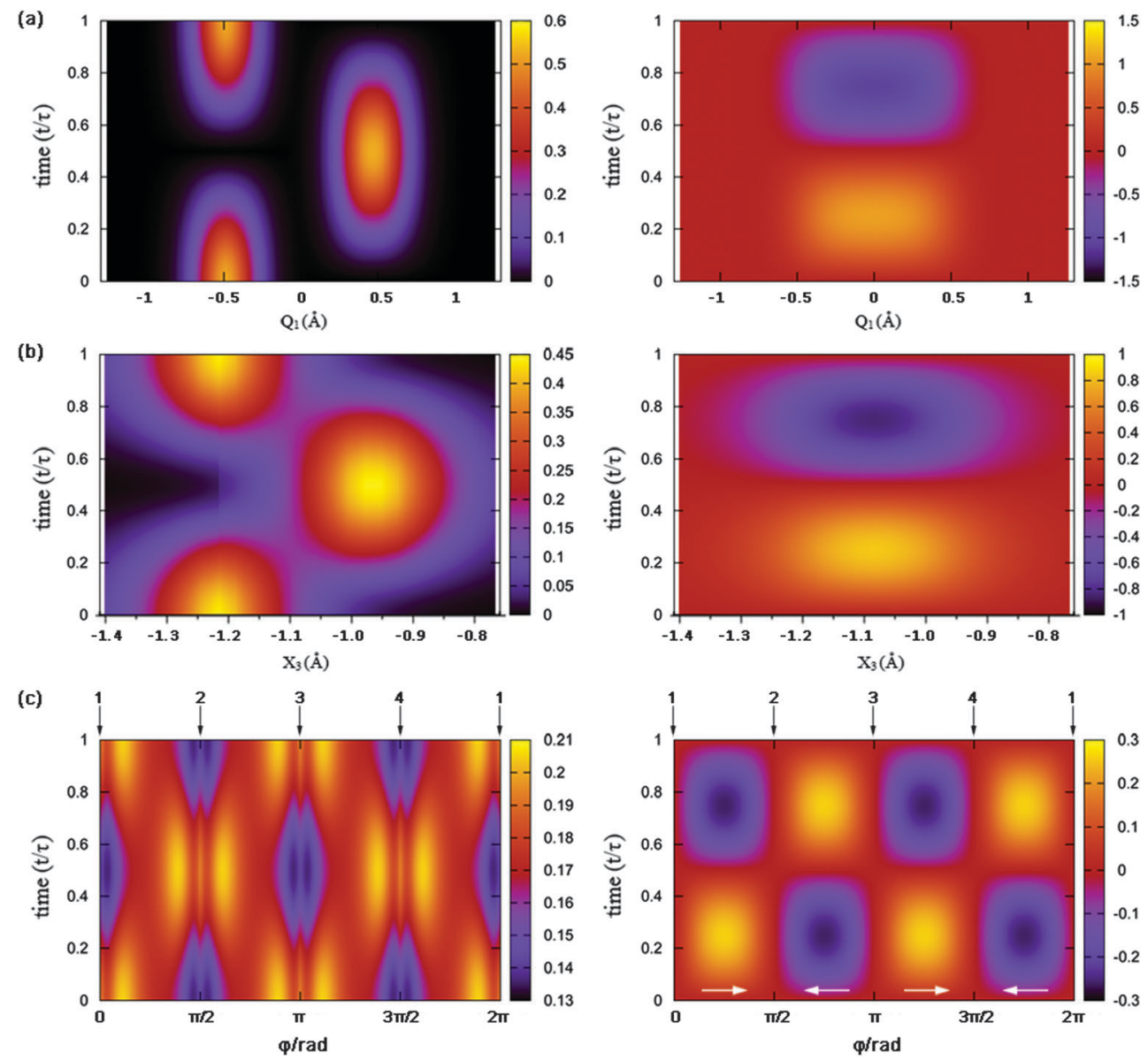

Fig. 13 Time evolution of probability densities (left) and fluxes (right) of nuclei at $Q_{1}$, or equivalently at $X_{3}$ (top, a), core electrons (middle, b) also at $Q_{1}$ or $X_{3}$ and valence electrons (bottom, $c$ ) at $\varphi$ during tunnelling isomerization of $\mathrm{B}_{4}$. Horizontal arrows in bottom right panel indicate fluxes of valence electrons, which alternate in direction in the four quadrants. Vertical arrows (bottom) labeled 1, 2, 3, and 4 indicate angular positions of nuclei B1, B2, B3, and B4 (cyclic).

not "like" to tunnel; they "prefer" to be at R or P rather than the potential barrier. But since quantum mechanics dictates that they must tunnel, ${ }^{73}$ they move through the barrier as fast as possible, thus maximizing the NFD at the barrier.

The nuclear flux observed at point $Q_{1}$ in the $1 \mathrm{D}$ internal frame can be related to the Cartesian components of fluxes of the individual nuclei in the NCM frame. According to eqn (51b), the flux of nucleus 3 in the $x$-direction observed at $X_{3}$, for example, is proportional to the flux observed at $Q_{1}=X_{3} /\left[\tilde{\mathbf{W}}^{-1}\right]_{3 x ; 1}$ :

$$
\left\langle j_{3 x}\left(X_{3}, t\right)\right\rangle=\frac{\mu_{1}}{M_{3}} \frac{\left[\tilde{\mathbf{W}}^{\mathrm{T}}\right]_{3 x ; 1}}{\left|\left[\tilde{\mathbf{W}}^{\mathrm{T}}\right]_{3 x ; 1}\right|}\left\langle j_{1}\left(Q_{1}=X_{3} /\left[\tilde{\mathbf{W}}^{\mathrm{T}}\right]_{3 x ; 1}, t\right)\right\rangle
$$

The proportionality constant in eqn (106), $c \equiv \frac{\mu_{1}}{M_{3}} \frac{\left[\tilde{\mathbf{W}}^{\mathrm{T}}\right]_{3 x ; 1}}{\left[\tilde{\mathbf{W}}^{-1}\right]_{3 x ; 1}}$, can be calculated directly from the matrix $\tilde{\mathbf{W}}$ (see eqn (100)). The result is $c=1$ (see Appendix C). It is worthwhile, however, to present an alternative, more intuitive derivation that avoids the substantial work of inverting $\tilde{\mathbf{W}}$. We exploit the fact that the NFD that accompanies coherent tunnelling in any direction can be written as a product of a spatial factor times the temporal factor, $(\pi / \tau) \sin (2 \pi t / \tau)$ (see Section 2.8). It therefore suffices to determine the constant $c$ at a single, arbitrary time $t$, and at single, arbitrary points of observation of the related coordinates $\left(Q_{1}\right.$ and $\left.X_{3}\right)$. The same constant then applies at all other times and related points of observation. For convenience we consider the time $t=\tau / 4$ (i.e., half of the time required for tunneling from $\mathrm{R}$ to $\mathrm{P}$ ), when the temporal factor achieves its maximum value $(\pi / \tau)$. We take the point of observation to be at the potential barrier halfway between $\mathrm{R}$ and $\mathrm{P}$ at $Q_{1}=Q_{1, \mathrm{~b}}=0$ or $X_{3, \mathrm{~b}}=0.5$ $\left(X_{3, \mathrm{R}}+X_{3, \mathrm{P}}\right)$, where the spatial factors attain their maxima. Because of the symmetry of the system, for this time and point of observation the nuclear yield is $1 / 2$ (i.e., the time integral of the flux associated with $Q_{1}$ at point of observation $Q_{1, \mathrm{~b}}$ over the interval [0, $\left.\tau / 4\right]$, yields the value $1 / 2$, as does the analogous time integral of the flux associated with $X_{3}$ ). Since the temporal factor is common to both, the spatial factors of the NFDs along $Q_{1}$ and $X_{3}$ at $Q_{1, \mathrm{~b}}$ and $X_{3, \mathrm{~b}}$ must also be the same. As a consequence, $c=1$. Strictly speaking, this analysis rests on the assumption that at $t=0$ and at $t=\tau / 2$, the wave packet is localized in the respective domains of $\mathrm{R}$ and $\mathrm{P}$, but this is an excellent approximation, in accord with the observed negligible values of the NPD at the barrier for $\mathrm{R}$ at $t=0$ and for $\mathrm{P}$ at $t=\tau / 2$. A generalization of this analysis is given in Appendix D. 
Hence, we have

$$
\left\langle j_{3 x}\left(X_{3}, t\right)\right\rangle=\left\langle j_{1}\left(Q_{1}, t\right)\right\rangle
$$

Further, by virtue of the symmetry of $Q_{1}$, we also have

$$
\left\langle j_{1}\left(Q_{1}, t\right)\right\rangle=-\left\langle j_{1 x}\left(X_{1}, t\right)\right\rangle=\left\langle j_{2 y}\left(Y_{2}, t\right)\right\rangle=-\left\langle j_{4 y}\left(Y_{4}, t\right)\right\rangle
$$

for the other nuclei.

Step 9

According to eqn (73), the 3D EPD of R(P) in the BOA is given by

$$
\begin{aligned}
& \left\langle\rho_{\mathrm{e}, \mathrm{NCM}}(\mathbf{x})\right\rangle_{\mathrm{BOA}, \mathrm{R}(\mathrm{P})} \\
& =\int \mathrm{d} Q_{1}\left|\chi_{\mathrm{R}(\mathrm{P})}\left(Q_{1}\right)\right|^{2}\left\{\sum_{i=1}^{N_{\mathrm{e}}} \prod_{j \neq i}^{N_{\mathrm{e}}} \int \mathrm{d} \mathbf{q}_{j}\left[\Phi_{0}\left(\mathbf{q} ; Q_{1}\right)\right]_{\mathbf{q}_{i}=\mathbf{x}}^{2}\right\} \\
& =\int \mathrm{d} Q_{1}\left\{\sum_{i=1}^{N_{\mathrm{e}}} \prod_{j \neq i}^{N_{\mathrm{e}}} \int \mathrm{d} \mathbf{q}_{j}\left[\Phi_{0}\left(\mathbf{q} ; Q_{1}\right)\right]_{\mathbf{q}_{i}=\mathbf{x}}^{2}\right\}\left\langle\rho_{1}\left(Q_{1}\right)\right\rangle_{\mathrm{BOA}, \mathrm{R}(\mathrm{P})}
\end{aligned}
$$

where the second line depends on eqn (103). Exploiting the form of $\Phi_{0}$ as a Slater determinant of canonical molecular spinorbitals (CMOs) (i.e., a product of a one-electron spatial function $\varphi_{k}\left(\mathbf{q}_{i} ; Q_{1}\right)$ and a spin eigenfunction, where $\left.k=1,2, \ldots, N_{\mathrm{e}} / 2\right)$, we can cast the quantity in braces in eqn (109) as

$$
\sum_{i=1}^{N_{\mathrm{e}}} \prod_{j \neq i}^{N_{\mathrm{e}}} \int \mathrm{d} \mathbf{q}_{j}\left[\Phi_{0}\left(\mathbf{q} ; Q_{1}\right)\right]_{\mathbf{q}_{i}=\mathbf{x}}^{2}=\rho\left(\mathbf{x}, Q_{1}\right)=2 \sum_{k=1}^{N_{\mathrm{e}} / 2} \rho_{k}\left(\mathbf{x}, Q_{1}\right)
$$

where

$$
\rho_{k}\left(\mathbf{x}, Q_{1}\right)=\left[\varphi_{k}\left(\mathbf{x} ; Q_{1}\right)\right]^{2}
$$

is the probability density of an electron in the $k$ th CMO (with the internal nuclear coordinate fixed at $Q_{1}$ ) at the point of observation $\mathbf{x}$ in the NCM frame. Note that, since the CMOs are normalized, we adopt the standard normalization

$$
\begin{aligned}
\int \mathrm{d} \mathbf{x} \rho\left(\mathbf{x}, Q_{1}\right) & =2 \sum_{k=1}^{N_{\mathrm{e}} / 2} \int \mathrm{d} \mathbf{x}\left[\varphi_{k}\left(\mathbf{x} ; Q_{1}\right)\right]^{2} \\
& =2 \sum_{k=1}^{N_{\mathrm{e}} / 2} 1=N_{\mathrm{e}}
\end{aligned}
$$

The CMOs for $\mathrm{B}_{4}$, computed at the present $(\operatorname{CCSD}(\mathrm{T})) /($ aug-ccpVTZ) level of quantum chemistry, are shown in Fig. 9 for R and $\mathrm{P}$ at $Q_{1}=Q_{1 \mathrm{R}}$ and $Q_{1}=Q_{1 \mathrm{P}}$. Four core CMOs $(k=1,2,3,4)$ can be distinguished from six valence CMOs $(k=5,6,7,8,9,10)$. Hence, the total EPD (at nuclear configuration $Q_{1}$ ) can be partitioned into core and valence contributions:

$$
\begin{array}{r}
\rho_{\text {core }}\left(\mathbf{x}, Q_{1}\right)=2 \sum_{k=1}^{4} \rho_{k}\left(\mathbf{x} ; Q_{1}\right) \\
\rho_{\text {val }}\left(\mathbf{x} ; Q_{1}\right)=2 \sum_{k=5}^{N_{\mathrm{e}} / 2=10} \rho_{k}\left(\mathbf{x}, Q_{1}\right)
\end{array}
$$

It follows from eqn (112) and (113) that

$$
\int \mathrm{d} \mathbf{x} \rho_{\text {core }}\left(\mathbf{x}, Q_{1}\right)=8
$$

$$
\int \mathrm{d} \mathbf{x} \rho_{\text {val }}\left(\mathbf{x}, Q_{1}\right)=12
$$

It is instructive to express the population density of core electrons as

$$
\rho_{\text {core }}\left(\mathbf{x}, Q_{1}\right)=\sum_{k=1}^{4} \rho_{\mathrm{c}, a}\left(\mathbf{x}, Q_{1}\right)=\sum_{k=1}^{4} 2\left[\varphi_{\mathrm{c}, \mathrm{a}}\left(\mathbf{x}, Q_{1}\right)\right]^{2}
$$

in terms of orbitals $\varphi_{\mathrm{c}, a}\left(\mathbf{x}, Q_{1}\right)$ that are localized at the nuclei $a$ $(=1,2,3,4)$ :

$$
\begin{aligned}
& \varphi_{\mathrm{c}, 1}\left(\mathbf{x} ; Q_{1}\right)=\frac{1}{\sqrt{2}}\left[\varphi_{1}\left(\mathbf{x} ; Q_{1}\right)-\varphi_{2}\left(\mathbf{x} ; Q_{1}\right)\right] \\
& \varphi_{\mathrm{c}, 2}\left(\mathbf{x} ; Q_{1}\right)=\frac{1}{\sqrt{2}}\left[\varphi_{3}\left(\mathbf{x} ; Q_{1}\right)-\varphi_{4}\left(\mathbf{x} ; Q_{1}\right)\right] \\
& \varphi_{\mathrm{c}, 3}\left(\mathbf{x} ; Q_{1}\right)=\frac{1}{\sqrt{2}}\left[\varphi_{1}\left(\mathbf{x} ; Q_{1}\right)+\varphi_{2}\left(\mathbf{x} ; Q_{1}\right)\right] \\
& \varphi_{\mathrm{c}, 4}\left(\mathbf{x} ; Q_{1}\right)=\frac{1}{\sqrt{2}}\left[\varphi_{3}\left(\mathbf{x} ; Q_{1}\right)+\varphi_{4}\left(\mathbf{x} ; Q_{1}\right)\right]
\end{aligned}
$$

Using eqn (109)-(111), we can express the EPDs associated with the core and valence contributions to $\mathrm{R}$ and $\mathrm{P}$ by

$$
\begin{gathered}
\left\langle\rho_{\mathrm{e}, \mathrm{NCM} ; \mathrm{cat}}(\mathbf{x})\right\rangle_{\mathrm{BOA}, \mathrm{R}}=\int_{Q_{1 l}}^{Q_{1 u}} \mathrm{~d} Q_{1} \rho_{\mathrm{cat}}\left(\mathbf{x}, Q_{1}\right)\left\langle\rho_{1}\left(Q_{1}\right)\right\rangle_{\mathrm{BOA}, \mathrm{R}} \\
\left\langle\rho_{\mathrm{e}, \mathrm{NCM} ; \mathrm{cat}}(\mathbf{x})\right\rangle_{\mathrm{BOA}, \mathrm{P}}=\int_{Q_{1 l}}^{Q_{1 u}} \mathrm{~d} Q_{1} \rho_{\text {cat }}\left(\mathbf{x}, Q_{1}\right)\left\langle\rho_{1}\left(Q_{1}\right)\right\rangle_{\mathrm{BOA}, \mathrm{P}}
\end{gathered}
$$

$$
\left\langle\Delta \rho_{\mathrm{e}, \mathrm{NCM} ; \mathrm{cat}}(\mathbf{x})\right\rangle_{\mathrm{BOA}}=\left\langle\rho_{\mathrm{e}, \mathrm{NCM} ; \mathrm{cat}}(\mathbf{x})\right\rangle_{\mathrm{BOA}, \mathrm{P}}-\left\langle\rho_{\mathrm{e}, \mathrm{NCM} ; \mathrm{cat}}(\mathbf{x})\right\rangle_{\mathrm{BOA}, \mathrm{R}}
$$

where the subscript "cat" (for category) refers to core, valence, or perhaps all, electrons. The lower and upper integration limits in eqn (117a) and (117b) are chosen so that the NPDs $\left\langle\rho_{1}\left(Q_{1}\right)\right\rangle_{\text {BOA,R }}$ and $\left\langle\rho_{1}\left(Q_{1}\right)\right\rangle_{\mathrm{BOA}, \mathrm{P}}$ are negligible outside the domain $\left[Q_{1 l}, Q_{1 u}\right]$. In practice we set $Q_{1 u}=-Q_{1 l}=1.3 \AA$. The resulting differences of the EPDs of the core electrons (occupying orbitals $\varphi_{\mathrm{c}, a}\left(\mathbf{x}, Q_{1}\right)$ ), which are localized at the nuclei (cat $=a=1,2,3,4$ ), are shown in Fig. 14. The difference of the EPD for all core electrons (cat $=$ core $)$, valence electrons $($ cat $=$ val $)$ and all electrons are shown in Fig. 10.

\section{Step 10}

From eqn (113)-(117) we deduce

$$
\left\langle\rho_{\mathrm{e}, \mathrm{NCM}}(\mathbf{x})\right\rangle_{\mathrm{BOA}, \mathrm{R}}=\sum_{a=1}^{4}\left\langle\rho_{\mathrm{e}, \mathrm{NCM} ; \mathrm{c}, a}(\mathbf{x})\right\rangle_{\mathrm{BOA}, \mathrm{R}}+\left\langle\rho_{\mathrm{e}, \mathrm{NCM} ; \mathrm{val}}(\mathbf{x})\right\rangle_{\mathrm{BOA}, \mathrm{R}}
$$




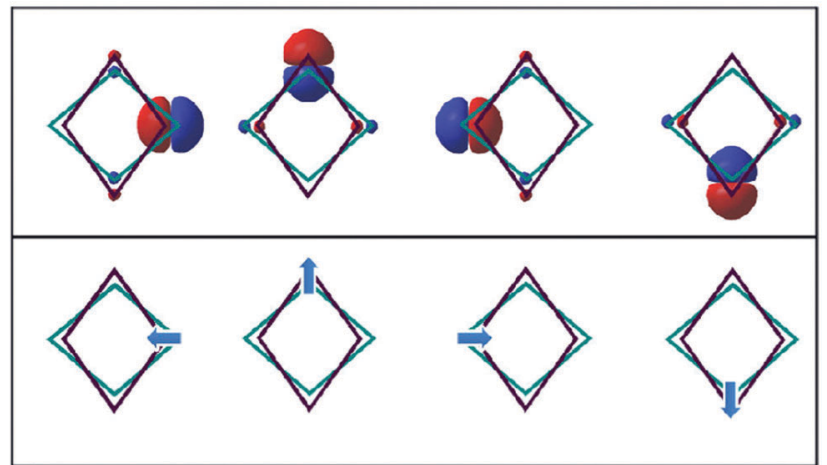

Fig. 14 Difference between one-electron probability densities of product $P$ and reactant $R$ for electrons in localized core orbitals (top) and main directions of electronic fluxes associated with the reaction $R \rightarrow P$ indicated by blue arrows (bottom). Positive and negative domains of electronic probability density difference are indicated by red and blue colors, respectively. Arrows represent electronic flux from centers of negative domains to centers of positive domains of the difference. Solid lines represent nuclear frames of rhombic R (blue-green) and $\mathrm{P}$ (red).

$$
\left\langle\Delta \rho_{\mathrm{e}, \mathrm{NCM}}(\mathbf{x})\right\rangle_{\mathrm{BOA}}=\sum_{a=1}^{4}\left\langle\Delta \rho_{\mathrm{e}, \mathrm{NCM} ; \mathrm{c}, a}(\mathbf{x})\right\rangle_{\mathrm{BOA}}+\left\langle\Delta \rho_{\mathrm{e}, \mathrm{NCM} ; \mathrm{val}}(\mathbf{x})\right\rangle_{\mathrm{BOA}}
$$

Combining eqn (118) and the BOA version of eqn (58a), we can express the time evolutions of the EPDs associated with specific core electrons (i.e., those occupying the orbital localized on nucleus labeled $a$ ) and (all) valence electrons as

$$
\begin{aligned}
& \left\langle\rho_{\mathrm{e}, \mathrm{NCM} ; \mathrm{c}, a}(\mathbf{x}, t)\right\rangle_{\mathrm{BOA}, \mathrm{R}} \\
& =\left\langle\rho_{\mathrm{e}, \mathrm{NCM} ; \mathrm{c}, a}(\mathbf{x})\right\rangle_{\mathrm{BOA}, \mathrm{R}}+\left\langle\Delta \rho_{\mathrm{e}, \mathrm{NCM} ; \mathrm{c}, a}(\mathbf{x})\right\rangle_{\mathrm{BOA}} \sin ^{2}\left(\frac{\pi t}{\tau}\right) \\
& \left\langle\rho_{\mathrm{e}, \mathrm{NCM} ; \mathrm{val}}(\mathbf{x}, t)\right\rangle_{\mathrm{BOA}, \mathrm{R}} \\
& =\left\langle\rho_{\mathrm{e}, \mathrm{NCM} ; \mathrm{val}}(\mathbf{x})\right\rangle_{\mathrm{BOA}, \mathrm{R}}+\left\langle\Delta \rho_{\mathrm{e}, \mathrm{NCM} ; \mathrm{val}}(\mathbf{x})\right\rangle_{\mathrm{BOA}} \sin ^{2}\left(\frac{\pi t}{\tau}\right)
\end{aligned}
$$

These formulas permit us to examine the EFDs that are due to the individual categories of electrons.

According to the general theory of Section 2, the main directions of the EFDs for electrons in core orbitals localized at the nuclei are from the negative to the positive lobes of $\left\langle\Delta \rho_{\mathrm{e}, \mathrm{NCM} ; \mathrm{c}, a}(\mathbf{x})\right\rangle_{\mathrm{BOA}, \mathrm{R}}($ i.e., along the $-x,+y,+x$ and $-y$ directions; see Fig. 14). These are parallel to the directions of the nuclear fluxes. This situation is pictured in Fig. 10b. Comparison with Fig. 10a shows that the core electrons flow synchronously with the nuclei, confirming our chemical intuition.

The main directions of the fluxes of valence electrons are deduced from the difference between the EPDs of $\mathrm{P}$ and $\mathrm{R}$, $\left\langle\Delta \rho_{\mathrm{e}, \mathrm{NCM} ; \mathrm{val}}(\mathbf{x})\right\rangle_{\mathrm{BOA}}$, which is shown in Fig. 10c. Symmetry implies that there are altogether four angular fluxes with alternating clockwise and counterclockwise directions in the cylindrical quadrants. The results for the core (Fig. 10b) and valence (Fig. 10c) electrons are summarized in Fig. 10d.

\section{Step 11}

The decomposition (eqn (118)) of the total EPD into contributions from core and valence electrons suggests the analogous decomposition of the EFD, and of the continuity equation (eqn (74), "beyond BOA"). Thus, we have

$$
\begin{aligned}
& \partial\left\langle\rho_{\mathrm{e}, \mathrm{NCM} ; \mathrm{c}, a}(\mathbf{x}, t)\right\rangle_{\mathrm{BOA}, \mathrm{R}} / \partial t+\nabla_{\mathbf{x}}\left\langle\left\langle j_{\mathrm{e}, \mathrm{NCM} ; \mathrm{c}, a}(\mathbf{x}, t)\right\rangle_{\mathrm{bBOA}, \mathrm{R}}=0\right. \\
& \partial\left\langle\rho_{\mathrm{e}, \mathrm{NCM} ; \mathrm{val}}(\mathbf{x}, t)\right\rangle_{\mathrm{BOA}, \mathrm{R}} / \partial t+\nabla_{\mathbf{x}} \cdot\left\langle j_{\mathrm{e}, \mathrm{NCM} ; \mathrm{val}}(\mathbf{x}, t)\right\rangle_{\mathrm{bBOA}, \mathrm{R}}=0
\end{aligned}
$$

for the core and valence electrons. We can also write the EFD as

$$
\begin{aligned}
\left\langle j_{\mathrm{e}, \mathrm{NCM}}(\mathbf{x}, t)\right\rangle_{\mathrm{bBOA}, \mathrm{R}}= & \sum_{a=1}^{4}\left\langle j_{\mathrm{e}, \mathrm{NCM} ; \mathrm{c}, a}(\mathbf{x}, t)\right\rangle_{\mathrm{bBOA}, \mathrm{R}} \\
& +\left\langle j_{\mathrm{e}, \mathrm{NCM} ; \mathrm{val}}(\mathbf{x}, t)\right\rangle_{\mathrm{bBOA}, \mathrm{R}}
\end{aligned}
$$

The individual components can then be reduced to 1D fluxes along arbitrary directions. Most interesting are the 1D fluxes along the main directions. For example, the 3D-to-1D reduction of the flux of the electrons in the core orbital localized at nucleus $a=3$ in the main direction (i.e., in the $x$-direction) yields

$$
\begin{aligned}
& \left\langle j_{\mathrm{e}, \mathrm{NCM} ; \mathrm{c}, 3,1 \mathrm{D}, x}(x, t)\right\rangle_{\mathrm{bBOA}} \\
& =-\int_{-\infty}^{x} \mathrm{~d} x^{\prime} \int_{-\infty}^{\infty} \mathrm{d} y^{\prime} \int_{-\infty}^{\infty} \mathrm{d} z^{\prime}\left\langle\Delta \rho_{\mathrm{e}, \mathrm{NCM} ; \mathrm{c}, 3}\left(\mathbf{r}^{\prime}\right)\right\rangle_{\mathrm{BOA}} \frac{\pi}{\tau} \sin \left(\frac{2 \pi t}{\tau}\right) \\
& =-\int_{-\infty}^{x} \mathrm{~d} x^{\prime}\left\langle\Delta \rho_{\mathrm{e}, \mathrm{NCM} ; \mathrm{c}, 3,1 \mathrm{D}, x}\left(x^{\prime}\right)\right\rangle_{\mathrm{BOA}} \frac{\pi}{\tau} \sin \left(\frac{2 \pi t}{\tau}\right)
\end{aligned}
$$

The difference between the reduced 1D EPDs of $\mathrm{R}$ and $\mathrm{P}$ for the core orbital centered at nucleus $3,\left\langle\Delta \rho_{\mathrm{e}, \mathrm{NCM} ; \mathrm{c}, 3,1 \mathrm{D}, x}\left(x^{\prime}\right)\right\rangle_{\mathrm{BOA}}$, is plotted in Fig. 12b. The resulting 1D flux (eqn (122)) of an electron in the core orbital localized at nucleus 3 is plotted in Fig. $12 \mathrm{c}$ at time $t=\tau / 4$. The time evolution of the probability density and the flux of an electron in the core orbital localized at nucleus 3 are illustrated by contour plots in Fig. 13b. Equivalent results, analogous to those given in eqn (107) and (108), are obtained for the EPDs and EFDs associated with the other core orbitals localized on nuclei 1, 2 and 4 .

Fig. 12b, c and 13a, b also allow the comparison between the probability densities and fluxes for nucleus 3 and the associated core electrons. The overall shapes are evidently the same, but the NPDs are more localized than the EPDs of core orbitals because of the larger mass of the nuclei compared with the electrons. The maximum values of the nuclear fluxes are therefore larger than those of the core electrons, which travel with the nuclei. The maxima of all fluxes occur at the barrier.

For the angular fluxes of the valence electrons, we use eqn (62a) and (63a) subject to two constraints: (i) tunnelling in the electronic ground state; (ii) conservation of $D_{2 \mathrm{~h}}$ symmetry during tunnelling. Accordingly, the angular flux of the valence electrons is zero at $\varphi=\varphi_{0}=0$ (and also at $\varphi=\pi / 2, \pi$ and $3 \pi / 2$ ); otherwise, either the $D_{2 \mathrm{~h}}$ symmetry would be broken or perhaps a ring current in an electronic excited state ${ }^{28}$ would be present. 
Thus, we set $\varphi_{0}=0$ in eqn (62a) and evaluate the angular flux of the valence electrons according to eqn (63a):

$$
\begin{aligned}
& \left\langle j_{\mathrm{e}, \mathrm{NCM} ; \mathrm{val}, 1 \mathrm{D}, \varphi}(\varphi, t)\right\rangle_{\mathrm{bBOA}, \mathrm{R}} \\
& =-\int_{0}^{\varphi} \mathrm{d} \varphi^{\prime} \int_{0}^{\infty} \mathrm{d} r^{\prime} r^{\prime} \int_{-\infty}^{\infty} \mathrm{d} z^{\prime}\left\langle\Delta \rho_{\mathrm{e}, \mathrm{NCM} ; \mathrm{val}}\left(\mathbf{r}^{\prime}\right)\right\rangle_{\mathrm{BOA}} \frac{\pi}{\tau} \sin \left(\frac{2 \pi t}{\tau}\right) \\
& =-\int_{0}^{\varphi} \mathrm{d} \varphi^{\prime}\left\langle\Delta \rho_{\mathrm{e}, \mathrm{NCM} ; \mathrm{val}, 1 \mathrm{D}, \varphi}\left(\varphi^{\prime}\right)\right\rangle_{\mathrm{BOA}} \frac{\pi}{\tau} \sin \left(\frac{2 \pi t}{\tau}\right)
\end{aligned}
$$

In practice the infinite limits on the integrations on $r^{\prime}$ and $z^{\prime}$ are replaced by sufficiently large finite values so that the integrals converge. The corresponding time evolution of the 1D angular population density of the valence electrons is obtained by analogous integration of the 3D densities in eqn (119b),

$$
\begin{aligned}
& \left\langle\rho_{\mathrm{e}, \mathrm{NCM} ; \mathrm{val}, 1 \mathrm{D}, \varphi}(\varphi, t)\right\rangle_{\mathrm{BOA}, \mathrm{R}} \\
& =\left\langle\rho_{\mathrm{e}, \mathrm{NCM} ; \mathrm{val}, 1 \mathrm{D}, \varphi}(\varphi)\right\rangle_{\mathrm{BOA}, \mathrm{R}}+\left\langle\Delta \rho_{\mathrm{e}, \mathrm{NCM} ; \mathrm{val}, 1 \mathrm{D}, \varphi}(\varphi)\right\rangle_{\mathrm{BOA}} \sin ^{2}\left(\frac{\pi t}{\tau}\right)
\end{aligned}
$$

The reduced 1D angular density difference of the valence electrons of $\mathrm{P}$ and $\mathrm{R},\left\langle\Delta \rho_{\mathrm{e}, \mathrm{NCM} ; \mathrm{val}, 1 \mathrm{D}, \varphi}(\varphi)\right\rangle_{\mathrm{BOA}}$, is plotted as a function of observation angle $\varphi$ in Fig. 15, together with the resulting angular flux $\left\langle j_{\mathrm{e}, \mathrm{NCM} ; \mathrm{val}, 1 \mathrm{D}, \varphi}(\varphi, t=\tau / 4)\right\rangle_{\mathrm{bBOA}, \mathrm{R}}$. The time evolutions of the angular densities and fluxes of the valence electrons are shown in Fig. 13c.

This section presents the first example of the perfect synchronicity of the CENFs during tunnelling isomerization. We have rationalized the counterintuitive result that all fluxes achieve their maximum values at the time $t=\tau / 4$, at the potential

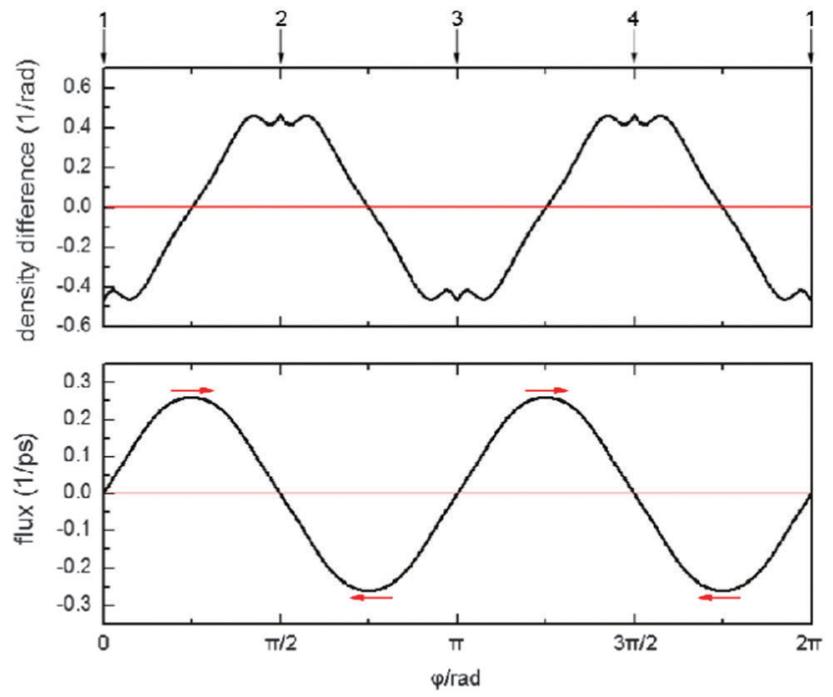

Fig. 15 Difference $\left\langle\Delta \rho_{\mathrm{e}, \mathrm{NCM} ; \mathrm{val}, 1 \mathrm{D}, \varphi}(\varphi)\right\rangle_{\mathrm{BOA}}$ between angular $(\varphi)$ components of one-electron densities of valence electrons for product $P$ and

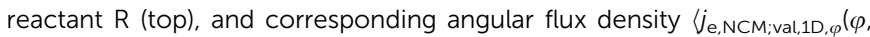
$t=\tau / 4)\rangle_{\mathrm{bBOA}, \mathrm{R}}$ during tunnelling isomerization of $\mathrm{B}_{4}$ (bottom). Vertical arrows indicate positions of nuclei 1, 2, 3, 4 (cyclic). Horizontal arrows $\rightarrow$ and $\leftarrow$ indicate anticlockwise and clockwise main directions of angular fluxes of valence electrons. barrier halfway between $\mathrm{R}$ and $\mathrm{P}$. Another key discovery is that the core electrons flow with the nuclei, whereas valence electrons may flow in oblique directions. In the specific case of $\mathrm{B}_{4}$, the opposing pairs of nuclei 1-3 and 2-4 flow in orthogonal directions. Apparently, the opposing nuclear fluxes drive angular fluxes of the valence electrons. This effect is reminiscent of that produced by two laser pulses with different, approximately perpendicular polarizations, which drive angular fluxes in ringshaped molecules. ${ }^{121,122}$

\section{Concerted electronic and nuclear fluxes during the Cope rearrangement of semibullvalene in the tunnelling domain}

The importance of tunnelling reactions in organic chemistry has been discussed recently. ${ }^{89,120,123-125}$ Here we focus on a typical example of a pericyclic reaction, specifically the degenerate 3,3 -sigmatropic shift, or Cope rearrangement ${ }^{126}$ of semibullvalene (SBV), as depicted in Fig. 16a.

Our choice of SVB is motivated by several considerations. From a general perspective, SBV and several of its derivatives have already served as touchstones for the study of various aspects of pericyclic reactivity: synthesis; ${ }^{127-131}$ spectroscopy; ${ }^{132-136}$ kinetics; ${ }^{132,137,138}$ electronic structure; ${ }^{129,130,134,135,139-148}$

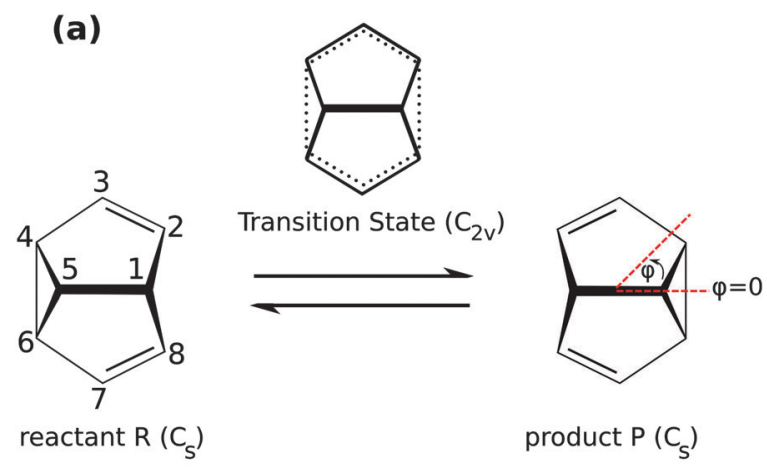

(b)

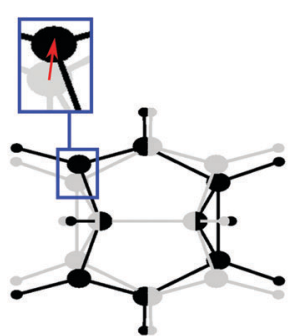

Fig. 16 (a) Bird's eye view of Cope rearrangement of semibullvalene (SBV). (b) Superposition of structures of reactant R (grey) and product $P$ (black) for scenario with $\mathrm{C} 1-\mathrm{C} 5$ bond parallel with $x$-axis. The $x-z$ plane is $C_{s}$ symmetry plane. Inset shows main direction of nuclear flux during tunnelling from $R$ to $P$, exemplarily for carbon nucleus $\mathrm{C} 4$. The vector (red) shows shift $\Delta_{\mathrm{C} 4} \mathbf{e}_{\mathrm{C} 4}$. 
thermochromicity; ${ }^{129-131,135,149}$ ab initio molecular dynamics; ${ }^{150}$ quantum dynamics of laser control. ${ }^{149,151-153}$ Thermochromicity of SBV, for example, is a consequence of the low potential barrier between the reactant $(\mathrm{R})$ and product $(\mathrm{P})$ and the large gap between the potential energy surfaces (PES) of the electronic ground and first excited states. ${ }^{135,143,144,151}$ Hence, the low barrier promotes tunnelling in SBV and it is safe to assume that tunnelling proceeds in the electronic ground state. Tunnelling is also enhanced by the rather low effective mass associated with the reaction coordinate, which has been estimated to be $\mu_{1}=\left(M_{\mathrm{C}}+M_{\mathrm{H}}\right) / 4 .{ }^{133}$

For the specific purpose of this perspective (i.e., the computation of CENFs), pericyclic reactions offer a model for the discovery of new phenomena ${ }^{18,26-28,44}$ and particularly for the quantitative interpretation of the traditional representation of electron transfer by curved arrows attached to Lewis structures. Many applications are documented in textbooks of organic, ${ }^{154-161}$ inorganic $^{162,163}$ and biochemistry. ${ }^{164,165}$ In general, the arrows indicate the breaking and making of chemical bonds through loss and gain of valence electrons. Those valence electrons that make the dominant contribution to this type of "pericyclic" electron transfer are called "pericyclic" electrons below; the corresponding EPD and EFD are also called "pericyclic". The term "pericyclic" suggests that the "pericyclic" electrons are transferred in a cyclical manner along the molecular perimeter, typically indicated by a cyclic (clockwise or counterclockwise) sequence of curved arrows in the Lewis structure. ${ }^{154-165}$ Previous theoretical investigations of pericyclic reactions have focussed on the Woodward-Hoffmann rules for the conservation of orbital symmetry, ${ }^{166}$ analyses of transition-state structures or intermediates, ${ }^{167-169}$ and assignments of concerted (or synchronous), as opposed to sequential, mechanisms. ${ }^{148,167-169}$ Through quantum simulations we have recently provided a quantitative interpretation of the arrows in Lewis structures describing the Cope rearrangement of SVB. ${ }^{27,44}$ We find that the "pericyclic" electrons do not flow strictly either clockwise or counterclockwise about the perimeter, but rather in clockwise and counterclockwise segments (referred to as "pincer-wise motion"). In other words, "pericyclic" reactions do not really occur "pericyclically," as it were. ${ }^{27,28,44}$ Moreover, we have determined the numbers of pericyclic electrons transferred during the "pericyclic" reactions, which turn out to be much smaller than assumed by the traditional rule ${ }^{154-165}$ (i.e., two electrons per curved arrow, or one per single-headed arrow symbolizing "pincer-wise motion"). In contrast to the previous investigations, ${ }^{27,44}$ which concern CENFs associated with states having energy well above the potential barrier, the present focus is on tunnelling states below the barrier, which are also considered in ref. 27.

SBV may be formally regarded as a derivative of 1,5-hexadiene, as a reference. The SBV comprises the (hexadiene) chain of six carbon atoms $\mathrm{C} 8=\mathrm{C} 7-\mathrm{C} 6-\mathrm{C} 4-\mathrm{C} 3=\mathrm{C} 2$ (reactant, $\mathrm{R}$, clockwise notation, (see Fig. 16)), susceptible to the Cope rearrangement to $\mathrm{C} 4=\mathrm{C} 3-\mathrm{C} 2-\mathrm{C} 8-\mathrm{C} 7=\mathrm{C} 6$ (product $\mathrm{P}$, keeping the labels of the carbon atoms), plus the bridge of two carbon atoms C1 and C5. On first glance the bridge may appear as a complication that makes the structure of SBV more unwieldy than the reference. For the present purpose of calculating CENFs during a pericyclic reaction, however, the bridge is quite helpful in that it keeps the configurations of $\mathrm{R}$ and $\mathrm{P}$ close to each other. As a consequence, the six relevant $\mathrm{C}$ atoms $(\mathrm{C} 8, \mathrm{C} 7, \mathrm{C} 6, \mathrm{C} 4, \mathrm{C} 3, \mathrm{C} 2)$ move over rather short distances during the tunnelling from $\mathrm{R}$ to P. This is illustrated in Fig. 16b, which depicts the superposition of $\mathrm{R}$ and $\mathrm{P}$ for the model with oriented $\mathrm{C}-\mathrm{C}$ bridge. The inset shows the rather short direct way of the carbon atom $\mathrm{C} 4$ from $\mathrm{R}$ to $\mathrm{P}$. Moreover, Fig. 16b indicates that the attached protons (with corresponding labels $\mathrm{H} 8, \mathrm{H} 7, \mathrm{H} 6, \mathrm{H} 4, \mathrm{H} 3, \mathrm{H} 2$ ) likewise travel only short distances from $\mathrm{R}$ to $\mathrm{P}$.

In summary, the Cope rearrangement of SBV serves as an ideal model system for the possible discovery of new features of CENFs during a pericyclic reaction in the tunnelling domain. The corresponding tunneling time, $\tau=1940 \mathrm{~s}$, has already been determined. ${ }^{123}$ (Note that in ref. 18, 27 and 44, $\tau=970 \mathrm{~s}$ is the time required for tunnelling just one way from $\mathrm{R}$ to $\mathrm{P}$.) Similar rather long, or even much longer (perhaps hours), tunnelling times have been measured recently for several other tunneling reactions. ${ }^{170,171}$ In the remainder of this section we derive the CENFs associated with the Cope rearrangement of SBV, following the same procedure used in Section 4 for tunneling isomerization of $\mathrm{B}_{4}$.

\section{Step 1}

We emphasize one of the fundamental results of Section 2: the Schrödinger equation yields synchronous EPDs and NPDs as well as EFDs and NFDs during coherent tunnelling isomerization of any molecule between symmetrically equivalent $\mathrm{R}$ and $\mathrm{P}$. This theorem provides an answer to the title question of ref. 148, "Electron-nuclear motion in the Cope rearrangement of SBV: ever synchronous?" The simple answer is, "yes, in the domain of coherent tunnelling!" The theorem also explains the specific result discovered in ref. 27, namely that the electronic flux out of the old single bond that is broken (C4-C6 in Fig. 16) is synchronous with the electronic flux into the new bond that is formed (C2-C8). That this result is not at all trivial is supported by the opposite asynchronous behavior of electronic fluxes for non-tunnelling states at energies above the potential barrier. ${ }^{27,44,148}$ The present theorem goes much beyond the result of ref. 27 , however, by proving that all CENFs associated with the tunnelling Cope rearrangement of SBV are perfectly synchronous.

\section{Step 2}

Fully optimized structures of the equivalent $\mathrm{R}$ and $\mathrm{P}$ for SVB shown in Fig. 16 are achieved by means of the second-order multi-reference perturbation theory using triple-zeta basis sets (RS2/cc-pVTZ), including a refinement at the RS3/cc-pVTZ ${ }^{172}$ and MRCI+dav/cc-pVTZ ${ }^{173-175}$ levels of quantum chemistry (see ref. 148 for the details). The results agree well with B3LYP density functional calculations. ${ }^{148,176,177}$ The B3LYP valence density is then partitioned by transformations into localized orbitals, according to the Pipek-Mezey method. ${ }^{178}$ The $C_{\mathrm{s}}$ symmetries of $\mathrm{R}$ and $\mathrm{P}$ suggest conservation of $C_{\mathrm{s}}$ symmetry during tunnelling. We assume that the NCM remains at the origin of the laboratory coordinate system. We also assume that the C1-C5 carboncarbon bridge is aligned with the $x$-axis (horizontal in Fig. 16), for example, by means of the methods of ref. 60-65. The $y$-axis is 
vertical and the $z$-axis is perpendicular to $\mathrm{C} 1-\mathrm{C} 5$ bridge, normal to the plane of the page in Fig. 16. Hence, the $x-z$ plane serves as $C_{\mathrm{s}}$ symmetry plane (perpendicular to the plane of the page in Fig. 16). Below, we shall also employ cylindrical coordinates $r$, $\varphi, z$ (see Fig. 16a), as in the treatment of coherent tunnelling of $\mathrm{B}_{4}$ in Section 4.

\section{Step 3}

The symmetry-adapted set of the main directions of the NFDs, which point from the positions of the nuclei of $\mathrm{R}$ to those of $\mathrm{P}$, are illustrated in Fig. 16b for C4. These main directions may be expressed in terms of unit vectors $\mathbf{e}_{a}$ in the direction of the distance $\mathbf{R}_{a, \mathrm{P}}-\mathbf{R}_{a, \mathrm{R}}=\Delta_{a} \mathbf{e}_{a}$, where $\Delta_{a}=\left|\mathbf{R}_{a, \mathrm{P}}-\mathbf{R}_{a, \mathrm{R}}\right|$. For example $\mathbf{e}_{4}=(0.078,0.780,0.626)$ and $\Delta_{a}=0.479 \AA$ for carbon nucleus C4 (see the inset in Fig. 16b). The position halfway between $\mathrm{R}$ and $\mathrm{P}$ corresponds to the barrier, $\mathbf{R}_{a, b}=\left(\mathbf{R}_{a, \mathrm{R}}+\mathbf{R}_{a, \mathrm{P}}\right) / 2$. The pathway of nucleus $a$ along the main direction from $\mathrm{R}$ to $\mathrm{P}$ can be expressed as $\mathbf{R}_{a, \lambda}=\mathbf{R}_{a, \mathbf{R}}+\lambda \Delta_{a} \mathbf{e}_{a}$, where $\lambda$ increases from 0 at $\mathbf{R}$ to 1 at $\mathrm{P}$. Alternatively, we can write $\mathbf{R}_{a, \lambda}=\mathbf{R}_{a, b}+\lambda \Delta_{a} \mathbf{e}_{a}$ where $\lambda$ increases from -0.5 at $\mathrm{R}$ to +0.5 at P. Conservation of $C_{\mathrm{s}}$ symmetry implies the equality of the main directions of various pairs of nuclei: $\mathbf{e}_{\mathrm{C} 1}=\mathbf{e}_{\mathrm{C} 5}=\mathbf{e}_{x}, \mathbf{e}_{\mathrm{C} 2}=\mathbf{e}_{\mathrm{C} 6}, \mathbf{e}_{\mathrm{C} 3}=\mathbf{e}_{\mathrm{C} 7}=-\mathbf{e}_{x}, \mathbf{e}_{\mathrm{C} 4}=\mathbf{e}_{\mathrm{C} 8}$ (see Fig. 16b). Similar equalities hold for the main directions of the attached protons, denoted $\mathbf{e}_{\mathrm{H} 1}=\mathbf{e}_{\mathrm{H} 5}$, etc., and for the centers of mass of the related $\mathrm{CH}$ bonds, denoted $\mathbf{e}_{1}=\mathbf{e}_{5}$, etc. Furthermore, the main directions of $\mathrm{C} 2$ and $\mathrm{C} 4$ (as well as $\mathrm{H} 2$ and $\mathrm{H} 4$ ) are mirror images of those of C8 and C6, respectively. Accordingly, symmetry implies the following equalities relating nuclear displacements during the tunnelling from $\mathrm{R}$ to $\mathrm{P}$

$$
\begin{gathered}
\Delta_{\mathrm{C} 1}=\Delta_{\mathrm{C} 5} \\
\Delta_{\mathrm{C} 2}=\Delta_{\mathrm{C} 4}=\Delta_{\mathrm{C} 6}=\Delta_{\mathrm{C} 8} \\
\Delta_{\mathrm{C} 3}=\Delta_{\mathrm{C} 7} \\
\Delta_{\mathrm{H} 1}=\Delta_{\mathrm{H} 5}, \text { etc. } \\
\Delta_{1}=\Delta_{5}=\Delta_{1,5} \\
\Delta_{2}=\Delta_{4}=\Delta_{6}=\Delta_{8}=\Delta_{2,4,6,8} \\
\Delta_{3}=\Delta_{7}=\Delta_{3,7}
\end{gathered}
$$

\section{Step 4}

The $3 N_{\mathrm{n}}-3=45$ internal nuclear mobile-type coordinates $Q_{1}$, $Q_{2}, \ldots, Q_{45}$ may be constructed in many ways. For example, one may employ the method developed in ref. 179. Basically, it consists of two steps. In the first one defines a suitable mobile for the eight carbon nuclei and eight attached protons according to the rules of Hirschfelder ${ }^{5-59}$ (e.g., the mobile illustrated in Fig. 17). Here the carbon nuclei and protons of the eight $\mathrm{CH}$ bonds are assembled in diatomic subsets (C1,H1), $(\mathrm{C} 2, \mathrm{H} 2), \ldots,(\mathrm{C} 8, \mathrm{H} 8)$ with corresponding $8 \times 3=24$ mobile coordinates $\mathbf{R}_{\mathrm{H} 1, X}-\mathbf{R}_{\mathrm{C} 1, X}, \mathbf{R}_{\mathrm{H} 1, Y}-\mathbf{R}_{\mathrm{C} 1, Y}, \ldots, \mathbf{R}_{\mathrm{H} 8, Z}-\mathbf{R}_{\mathrm{C} 8, Z}$. This is indicated by the corresponding labels $1,2, \ldots, 8$ on the horizontal lines connecting pairs of atoms $(\mathrm{C} 2, \mathrm{H} 2)$, etc. in Fig. 17. At the next level of the mobile the diatomic subsets 1 $(\mathrm{C} 2, \mathrm{H} 2), 3(\mathrm{C} 4, \mathrm{H} 4)$ and $5(\mathrm{C} 3, \mathrm{H} 3)$ are connected with their respective mirror images $2(\mathrm{C} 8, \mathrm{H} 8), 4(\mathrm{C} 6, \mathrm{H} 6)$ and $6(\mathrm{C} 7, \mathrm{H} 7)$ in the $x-z$ plane and the bridge pairs $7(\mathrm{C} 5, \mathrm{H} 5)$ and $8(\mathrm{C} 1, \mathrm{H} 1)$ are joined to form four subsets of two diatomics each (labelled 9, 10, 11, and 12 in Fig. 17). The corresponding $4 \times 3=12$ additional mobile coordinates are the $x$ - $y$ - and $z$-Cartesian components of the distances from the center of mass of one $\mathrm{CH}$ bond to that of its symmetrically related partner. Analogous constructions of the remaining $45-24-12=9=3 \times 3$ mobile coordinates corresponding to Cartesian components of distances between centers of mass of successively larger subsets of nuclei can be deduced from Fig. 17. We finally arrive at the highest level labelled 16, which corresponds to the coordinates $S_{x}, S_{y}, S_{z}$ of the NCM. The $48 \times 48$ matrix $\tilde{\mathbf{W}}$ for the transformation from the laboratory Cartesian coordinates to mobile coordinates, which is given explicitly in ref. 179 , is block-diagonal, comprising three identical $16 \times 16$ blocks correlating with the $x$-, $y$ - and $z$-components.

In the second step we retain the $3 \mathrm{NCM}$ and 24 (internal) mobile coordinates for the diatomic subsets $(\mathrm{C} 1, \mathrm{H} 1),(\mathrm{C} 2, \mathrm{H} 2)$, and so on, but replace the remaining $12+9$ internal mobile coordinates by a linear combination, $Q_{1}$, that accounts for the motions of all nuclei during tunnelling from $\mathrm{R}$ to $\mathrm{P}$. In practice, $Q_{1}$ is constructed by a sequence of unitary transformations of $\tilde{\mathbf{W}} \cdot{ }^{179}$ For the present purpose, however, it is not necessary to carry out these transformations. Rather we can take a straightforward shortcut to $Q_{1}$ (shown immediately below in Step 5) that exploits the conservation of $C_{\mathrm{s}}$ symmetry during tunneling from $\mathrm{R}$ to $\mathrm{P}$.

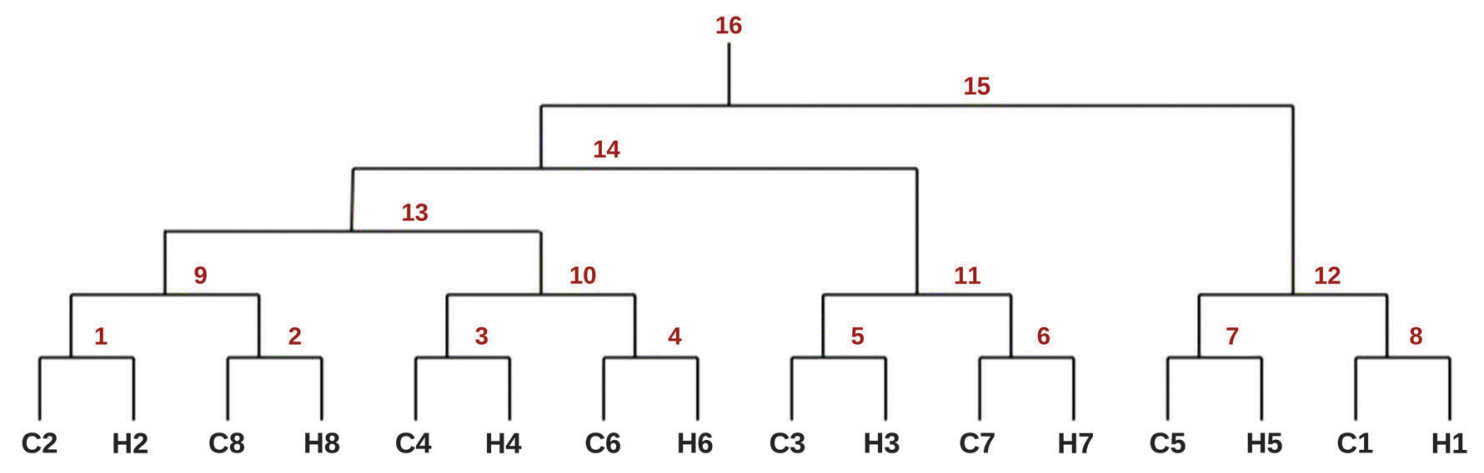

Fig. 17 Mobile for carbon nuclei $\mathrm{C} 1, \ldots, \mathrm{C} 8$ and attached protons $\mathrm{H} 1, \ldots, \mathrm{H} 8$ of SBV. 


\section{Step 5}

To derive the $1 \mathrm{D}$ model for tunnelling from $\mathrm{R}$ to $\mathrm{P}$ along the coordinate $Q_{1}$, we construct the mobile according to the sequence of steps outlined in Step 4, but we also introduce the following $3+8 \times 3=27$ constraints:

$$
\begin{gathered}
S_{x}=S_{y}=S_{z}=0 \\
\Delta_{\mathrm{Ca}} \mathbf{e}_{\mathrm{Ca}} \cong \Delta_{\mathrm{Ha}} \mathbf{e}_{\mathrm{Ha}} \cong \Delta_{\mathrm{a}} \mathbf{e}_{\mathrm{a}}=\mathbf{e}_{\mathrm{a}}\left(M_{\mathrm{C}} \Delta_{\mathrm{Ca}}+M_{\mathrm{H}} \Delta_{\mathrm{Ha}}\right) /\left(M_{\mathrm{C}}+M_{\mathrm{H}}\right)
\end{gathered}
$$

Fig. 16b shows that this approximation is reasonable. The shifts of the centers of mass of the $\mathrm{CH}$ bonds, $\Delta_{a}$, are fixed by the structures of $\mathrm{R}$ and $\mathrm{P}$, which are determined in Step 2. As a consequence, $Q_{1}$ should describe motions of all nuclei subject to the constraints (eqn (126)). Hence, $Q_{1}$ is a linear combination of the remaining 21 mobile coordinates, which in turn are linear combinations of the centers of mass of the $\mathrm{CH}$ bonds. A derivation analogous to that leading to eqn (99c) for $\mathrm{B}_{4}$ then yields an expression for the shift of $Q_{1}$ from $\mathrm{R}$ to $\mathrm{P}$ as a sum of the shifts of those centers of mass, namely

$$
\Delta Q_{1}=\sum_{a=1}^{8} \Delta_{a}=4 \Delta_{2,4,6,8}+2 \Delta_{3,7}+2 \Delta_{1,5}
$$

The second equality in eqn (127) is a consequence of the conservation of $C_{\mathrm{s}}$ symmetry. Moreover, an Ehrenfest-type argument, analogous to the one that leads to eqn (99b), gives

$$
\begin{aligned}
\mu_{1} \Delta Q_{1}^{2} & =\sum_{a=1}^{8}\left(M_{\mathrm{C}} \Delta_{\mathrm{Ca}}{ }^{2}+M_{\mathrm{H}} \Delta_{\mathrm{Ha}}{ }^{2}\right) \cong \sum_{a=1}^{8}\left(M_{\mathrm{C}}+M_{\mathrm{H}}\right) \Delta_{a}{ }^{2} \\
& =\left(M_{\mathrm{C}}+M_{\mathrm{H}}\right)\left(4 \Delta_{2,4,6,8}{ }^{2}+2 \Delta_{3,7^{2}}+2 \Delta_{1,5}{ }^{2}\right)
\end{aligned}
$$

The second and third equalities in eqn (128) are consequences of the approximation (eqn (126b)) and the conservation of $C_{\mathrm{s}}$ symmetry, which implies the relations in eqn (125). From eqn (127) and (128) we obtain the reduced mass associated with $Q_{1}$

$$
\mu_{1}=\frac{\left(M_{\mathrm{C}}+M_{\mathrm{H}}\right)\left(4 \Delta_{2,4,6,8}{ }^{2}+2 \Delta_{3,7^{2}}+2 \Delta_{1,5}{ }^{2}\right)}{\left(4 \Delta_{2,4,6,8}+2 \Delta_{3,7}+2 \Delta_{1,5}\right)^{2}}
$$

Inserting the values $\Delta_{2,4,6,8}=0.479 \AA, \Delta_{3,7}=0.089 \AA$ and $\Delta_{1,5}=$ $0.012 \AA$ obtained from Step 2, we get

$$
\mu_{1}=0.208\left(M_{\mathrm{C}}+M_{\mathrm{H}}\right)
$$

The reduced mass $\mu_{1}$ is clearly dominated by the large shifts $\Delta_{2,4,6,8}$ associated with the movements of the nuclei that participate in breaking the old C4-C6 bond of R and making the new $\mathrm{C} 2-\mathrm{C} 8$ bond of $\mathrm{P}$; all other shifts are much smaller. Neglecting those other shifts, we have the approximation

$$
\mu_{1} \cong\left(M_{\mathrm{C}}+M_{\mathrm{H}}\right) / 4
$$

which is derived in ref. 133 by exclusive consideration of the motions of the nuclei that participate in bond breaking and bond making, and is also used in ref. 18, 27 and 44. Finally, from eqn (128) and (129c) we obtain

$$
\Delta Q_{1}=2.072 \AA
$$

The convention $Q_{1, b}=0$ at the barrier implies that the collective internal nuclear coordinate $Q_{1}$ is given by the expression

$$
Q_{1}=\lambda \Delta Q_{1} / 2
$$

which is related to the motions of the individual nuclei by

$$
\mathbf{R}_{a} \cong \mathbf{R}_{a, b}+\lambda \Delta_{a} \mathbf{e}_{a}
$$

The special values $\lambda_{\mathrm{R}}=-0.5$ and $\lambda_{\mathrm{P}}=0.5$ give the coordinates corresponding to $\mathrm{R}$ and $\mathrm{P}$, respectively.

We note that the assumed orientation of the C1-C5 bridge parallel with the $x$-axis implies that during tunneling the molecule rotates about the $y$-axis by a few degrees. If we fixed the overall rotations instead, then the C1-C5 axis would rotate about the $y$-axis by a few degrees, thus compensating the opposite small-angle rotations of the "ring" of carbon nuclei $\mathrm{C} 2-\mathrm{C} 3-\mathrm{C} 4-\mathrm{C} 6-\mathrm{C} 7-\mathrm{C} 8$. Either choice leads to the same conclusions.

\section{Step 6}

The tunnelling time $\tau=1940 \mathrm{~s}$ for Cope rearrangement of SBV is taken from ref. 123 .

\section{Step 7}

The NPDs of $\mathrm{R}$ and $\mathrm{P}$, their difference, and the time evolution of the NPDs during tunnelling isomerization of SBV from $\mathrm{R}$ to $\mathrm{P}$ and back are computed for the $1 \mathrm{D}$ model, in a fashion entirely analogous to that of Step 7 of Section 4 for the tunnelling isomerization of $\mathrm{B}_{4}$. The resulting difference of the NPDs of $\mathrm{P}$ and $\mathrm{R}$ is shown in Fig. 18a, embedded in the double well potential $V\left(Q_{1}\right)$, where $Q_{1}=\lambda \Delta Q_{1} / 2$ (see eqn (131a)). The time evolution of the NPD is illustrated by the contour plot in Fig. 18b. These results for SBV are entirely analogous to those for $\mathrm{B}_{4}$ (compare Fig. 18a and b with Fig. 12b and 13 (top left panel), respectively).

\section{Step 8}

The calculation of the NFDs along the main directions during tunnelling isomerization of SBV is also entirely analogous to that described in Step 8 of Section 4 . The result for SBV is shown as a contour plot in Fig. 18c, which is analogous to Fig. 13 (top right panel) for $\mathrm{B}_{4}$. Fig. $18 \mathrm{c}$ confirms the counterintuitive effect that was first reported in ref. 18: the maximum values of the nuclear fluxes are observed at the potential barrier, halfway between $\mathrm{R}$ and $\mathrm{P}$.

\section{Step 9}

The EPDs of $\mathrm{R}$ and $\mathrm{P}$ and their difference, which are evaluated by the methods described in Step 2, are partitioned into different categories ("cat") based on the localized molecular orbitals. ${ }^{178}$ We distinguish the core electrons (localized at the carbon nuclei $a=1,2, \ldots, 8(\mathrm{cat}=\mathrm{c}, a)$ ), six pericyclic electrons that contribute to pericyclic electron transfer, correlating with changes in the Lewis structures of $\mathrm{R}$ and $\mathrm{P}$ (cat = peri), and the other valence electrons (cat = oval) that account, for example, for the $\mathrm{CH}$ bonds not associated with changes in the Lewis structures of $\mathrm{R}$ and $\mathrm{P} .{ }^{44}$ 
(a)
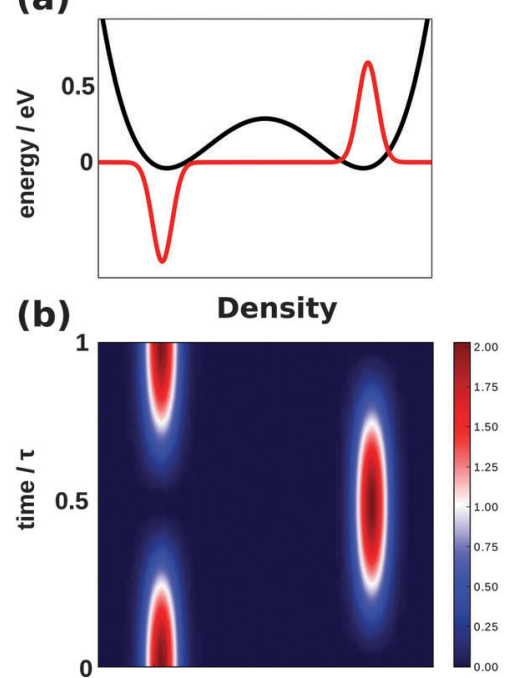

(c) Flux Density

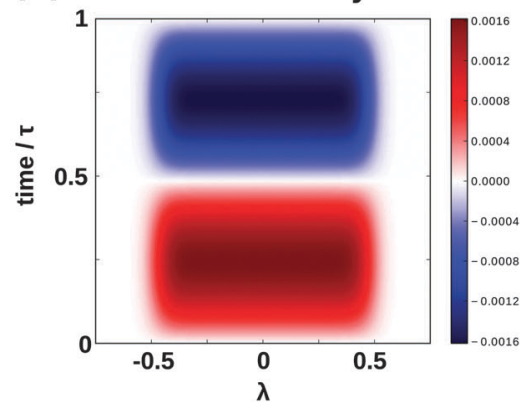

Fig. 18 (a) Double well potential for Cope rearrangement of SBV. Horizontal line indicating mean energy of lowest tunneling doublet serves as base-line for continuous curve, which represents difference of nuclear densities of product $\mathrm{P}$ (right) and reactant $\mathrm{R}$ (left). Variable $\lambda$ (abscissa) assumes special values $\lambda=-0.5,0.0$, and 0.5 at bottom of potential well for $\mathrm{R}$, at potential barrier, and at bottom of potential well for $\mathrm{P}$, respectively. (b) Contour plot for time evolution of nuclear probability density from $\mathrm{R}$ at $t=0$ to $\mathrm{P}$ at $t=\tau / 2$ and back to $\mathrm{R}$ at $t=\tau$, during coherent tunneling. Tunneling time $\tau=1940 \mathrm{~s}$ is taken from ref. 123. (c) Contour plot of corresponding nuclear flux density, with maximum value at time $t=\tau / 4$, at potential barrier.

\section{Step 10}

The symmetry-adapted main directions of the EFDs during tunneling isomerization of SBV are determined separately for each category of electrons, as in Step 10 of Section 4 for the tunneling isomerization of $\mathrm{B}_{4}$. We consider first the electrons in core orbitals localized at the carbon nuclei. For a given nucleus $a$, the corresponding electron densities $\left\langle\rho_{\mathrm{e}, \mathrm{NCM} ; \mathrm{c}, a}(x)\right\rangle_{\mathrm{BOA}, \mathrm{R}}$ of $\mathrm{R}$ and $\left\langle\rho_{\mathrm{e}, \mathrm{NCM} ; \mathrm{c}, a}(x)\right\rangle_{\mathrm{BOA}, \mathrm{P}}$ of $\mathrm{P}$ are localized and centered at $\mathbf{R}_{a, \mathrm{R}}$ and at $\mathbf{R}_{a, \mathrm{P}}$. The localized core EFDs therefore point from $\mathbf{R}_{a, \mathrm{R}}$ to $\mathbf{R}_{a, \mathrm{P}}$ (i.e., in the same directions $\mathbf{e}_{\mathrm{Ca}}$ as the shift in carbon nuclei) in tunnelling from $\mathrm{R}$ to $\mathrm{P}$. This confirms an important result of Step 10 of Section 4, i.e., that the core electrons flow with the nuclei.

Next we focus on the symmetry-adapted main directions of the EFDs of pericyclic electrons. Some important properties of the EFDs of the other valence electrons have already been reported in ref. 44. These EFDs point from centers of the negative regions of the population density difference $\left\langle\Delta \rho_{\text {e,NCM;peri }}(\mathbf{x})\right\rangle_{\text {BOA }}=\left\langle\rho_{\text {e,NCM;peri }}(\mathbf{x})\right\rangle_{\text {BOA }, \mathrm{P}}-\left\langle\rho_{\mathrm{e}, \mathrm{NCM} \text {;peri }}(\mathbf{x})\right\rangle_{\text {BOA }, \mathrm{R}}$ to positive regions. These centers of positive or negative difference are arranged on the molecular perimeter, essentially along the ring of carbon nuclei $\mathrm{C} 2-\mathrm{C} 3-\mathrm{C} 4-\mathrm{C} 6-\mathrm{C} 7-\mathrm{C} 8-\mathrm{C} 2$ about the $z$-axis. The way along this ring may be mapped onto the cylindrical coordinate $\varphi$. Integrating the pericyclic product and reactant densities $\left\langle\rho_{\mathrm{e}, \mathrm{NCM} \text {;peri }}(\mathbf{x})\right\rangle_{\text {BOA,P }}$ and $\left\langle\rho_{\mathrm{e}, \mathrm{NCM} \text {;peri }}(\mathbf{x})\right\rangle_{\text {BOA,R }}$, as well as their difference $\left\langle\Delta \rho_{\text {e,NCM;peri }}(\mathbf{x})\right\rangle_{\text {BOA }}$, over the complementary cylindrical coordinates $r$ and $z$ (as in eqn (123)), we obtain the corresponding 1D angular pericyclic electron densities $\left\langle\rho_{\mathrm{e}, \mathrm{NCM} ; \text { peri,1D }, \varphi}(\varphi)\right\rangle_{\mathrm{BOA}, \mathrm{P}},\left\langle\rho_{\mathrm{e}, \mathrm{NCM} ; \text { peri,1D }, \varphi}(\varphi)\right\rangle_{\mathrm{BOA}, \mathrm{R}}$, as well as their difference $\left\langle\Delta \rho_{\mathrm{e}, \mathrm{NCM} ; \text { peri,1D, } \varphi}(\varphi)\right\rangle_{\mathrm{BOA}}$. These are illustrated in Fig. 19a. The symmetry-adapted main angular flux directions point from the negative to the positive domains of the pericyclic EPD difference. Apparently there are six major domains with alternating positive and negative signs of $\left\langle\Delta \rho_{\mathrm{e}, \mathrm{NCM} \text {;peri,1D, } \varphi}(\varphi)\right\rangle_{\mathrm{BOA}}$. The nodes between these domains are located close to the positions of the carbon nuclei C2, C3, C4, C6, C7, C8. From this intermediate result we may already speculate that Cope rearrangement of SBV in the tunnelling domain should involve six domains of pericyclic angular EFDs with alternating directions. This conjecture is supported by comparison of Fig. 19a showing the difference of the angular pericyclic electron densities of $\mathrm{R}$ and $\mathrm{P}$ of SBV with the upper panel of Fig. 15 showing the difference of the angular densities of the valence electrons of $\mathrm{R}$ and $\mathrm{P}$ of $\mathrm{B}_{4}$. The latter has four domains with alternating signs and nodes at angular positions halfway between the boron nuclei, thus giving rise to four angular EFDs with alternating directions during tunneling isomerization of $\mathrm{B}_{4}$ (see the lower panel of Fig. 15).

\section{Step 11}

We determine the EFDs of different categories of electrons along their main directions during tunnelling isomerization of SBV by integrating the differences of the corresponding electronic densities of $\mathrm{P}$ and $\mathrm{R}$ determined in Step 10 over the appropriate complementary coordinates to obtain the 1D flux. Again, this step is entirely analogous to its counterpart for the tunnelling isomerization of $\mathrm{B}_{4}$ (see Section 4).

We consider first the EFDs of core electrons. As for $\mathrm{B}_{4}$, the underlying population density differences of $\mathrm{P}$ and $\mathrm{R}$ resemble those for the nuclei (i.e., they have bell-shaped negative and positive distributions centered at positions of the nuclei a corresponding to $\mathrm{R}$ and $\mathrm{P}$ ). The EPDs are slightly broader, with slightly lower peaks compared with the NPDs, essentially because of the greater delocalization of core electrons compared with the nuclei. Integrating these density differences (multiplied by -1 ) over the complementary coordinates yields the corresponding characteristic symmetric shapes of the (beyond BOA) fluxes (1D EFDs) of core electrons during tunneling isomerization of SVB from $\mathrm{R}$ to $\mathrm{P}$, analogous to those for $\mathrm{B}_{4}$. We note in passing that ref. 44 presents selected results for the fluxes of core electrons along angular directions. It is emphasized, however, that these angular directions are not the main directions of the fluxes of core electrons. Hence, the results for core electrons in ref. 44 are less relevant compared with the present results. 


\section{(a)}

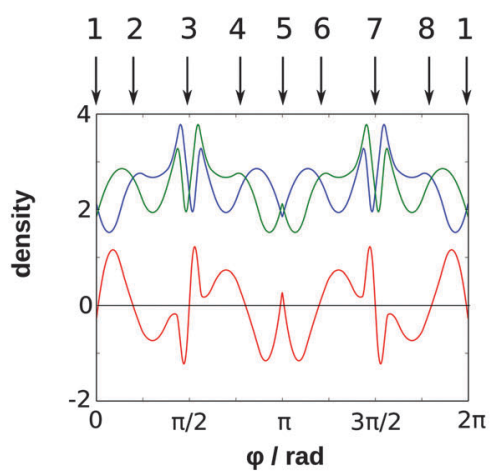

(b)

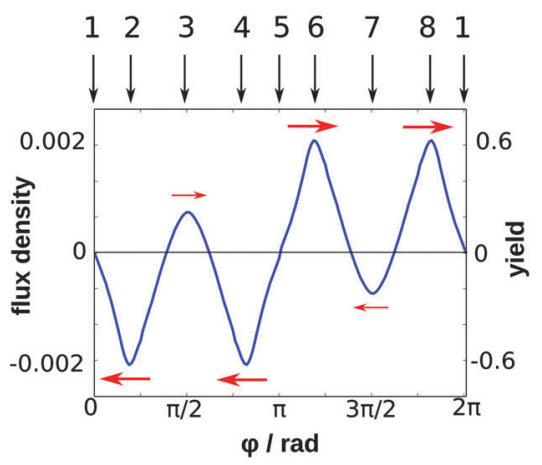

(c)

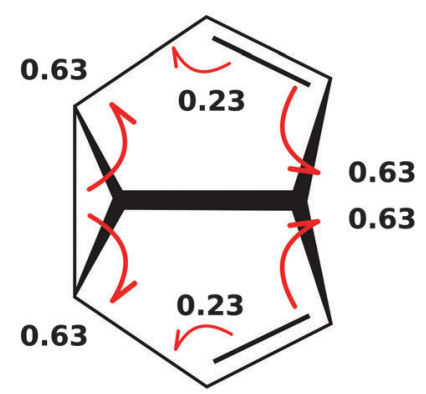

Fig. 19 (a) Angular probability densities of pericyclic electrons that contribute to changes in Lewis structures of SBV (see Fig. 16) upon Cope rearrangement in the domain of coherent tunnelling from reactant $(R$, blue) to product $(P$, green), and their difference $(P-R$, red). Vertical arrows with cyclic labels $1,2, \ldots, 8,1$ indicate angular positions of nuclear centers of mass of $\mathrm{CH}$ bonds halfway between $\mathrm{R}$ and $\mathrm{P}$. (b) Corresponding angular flux density of pericyclic electrons. Horizontal arrows $(\rightarrow$ and $\leftarrow$ ) indicate clockwise and counterclockwise fluxes. (c) Lewis structure of $\mathrm{R}$ with curved arrows showing alternating ("pincer-type") angular fluxes of pericyclic electrons. Numbers at arrows specify maximum numbers of pericyclic electrons transferred by these fluxes.

Finally, we examine the angular EFDs of pericyclic electrons. According to the general theory of Section 2.8, a prerequisite for the calculation of any angular EFDs $\left\langle j_{\mathrm{e}, \mathrm{NCM} ; 1 \mathrm{D}, \varphi}(\varphi, t)\right\rangle$ is knowledge of its value $\left\langle j_{\mathrm{e}, \mathrm{NCM} ; 1 \mathrm{D}, \varphi}\left(\varphi_{0}, t\right)\right\rangle$ for at least one specific angle $\varphi_{0}$ (see eqn (62a) and (63a)). In the case of tunnelling isomerization of SBV, we employ conservation of $C_{\mathrm{s}}$ symmetry to deduce the result $\left\langle j_{\mathrm{e}, \mathrm{NCM} ; 1 \mathrm{D}, \varphi}\left(\varphi_{0}=0, t\right)\right\rangle=0$, for all categories of electrons, including pericyclic electrons. Otherwise the electronic fluxes would either break $C_{\mathrm{s}}$ symmetry or they would have contributions from ring currents in electronic excited states. $^{28}$ Because of the symmetry of the system, the condition $\left\langle j_{\mathrm{e}, \mathrm{NCM} ; 1 \mathrm{D}, \varphi}\left(\varphi_{0}=0, t\right)\right\rangle=0$ implies that the angular fluxes of all categories of electrons must also vanish at $\varphi_{0}{ }^{\prime}=\pi$. Below we point out two additional pairs of special angles for zero pericyclic electron fluxes, which can be expessed as symmetry-adapted sets $\left\{ \pm \varphi_{0}{ }^{\prime \prime}\right.$ and $\left.\pi \pm \varphi_{0}{ }^{\prime \prime}\right\}$. Although their existence can be predicted by means of the selection rule for the directions of pericyclic angular fluxes, ${ }^{28}$ there is no a priori way to deduce the value of $\varphi_{0}{ }^{\prime \prime}$. Using the angle $\varphi_{0}=0$, we apply eqn (123) to evaluate the pericyclic electron flux $\left\langle j_{\mathrm{e}, \mathrm{NCM} \text {;peri,1D, } \varphi}(\varphi, t)\right\rangle_{\mathrm{bBOA}, \mathrm{R}}$ in the "beyond the BOA" approximation. The calculation is completely parallel with that of the angular fluxes of valence electrons during tunneling isomerization of $\mathrm{B}_{4}$. The result is shown in Fig. 20 as contour plot, together with a contour plot for the time evolution of the pericyclic EPD $\left\langle\rho_{\mathrm{e}, \mathrm{NCM} \text {;peri,1D, } \varphi}(\varphi, t)\right\rangle_{\mathrm{bBOA}, \mathrm{R}}$ (compare with the analogous expression in eqn (124) for $\mathrm{B}_{4}$ ). The vertical arrows in Fig. 20 indicate the angular positions of the carbon nuclei, in cyclic order $1,2, \ldots, 8,1$, at the potential barrier halfway between $\mathrm{R}$ and $\mathrm{P}$. The angular pericyclic flux during tunneling from $\mathrm{R}$ to $\mathrm{P}$ apparently consists of six parts with alternating a, c, a, c, a, c directions, indicated by horizontal arrows $\rightarrow$ (clockwise, a) or $\leftarrow$ (counterclockwise, c). Extreme values are attained at $t=\tau / 4$ (i.e., at half the tunnelling at time from $\mathrm{R}$ to $\mathrm{P}$ ). The angular positions of the extreme values of the fluxes coincide with the positions of the carbon nuclei $\mathrm{C} 2, \mathrm{C} 3$, C4, C6, C7, C8 at the barrier, which can be seen in the plot of Fig. $19 \mathrm{~b}$ on a finer scale. Apparently, conservation of $C_{\mathrm{s}}$ symmetry implies equal absolute values of the maximum fluxes of pericyclic electron fluxes through observation planes at the barrier positions of nuclei C2 (a), C4 (a), C6 (c) and C8 (c). Smaller local maximum values are obtained at C3 (c) and C7 (a). The corresponding arrows are mapped in Fig. 19c onto the series of six curved arrows with alternating a, c, a, c, a, c directions embedded in the Lewis structure of $\mathrm{R}$. In contrast to the diagrams in most textbooks on organic chemistry, they do not show the cyclic $\mathrm{c}$ or ac patterns, but rather a pincer-type pattern of alternating a and ac parts.

In order to quantify the curved arrows in the Lewis structure of $\mathrm{R}$ symbolizing pericyclic electronic fluxes during tunnelling from $\mathrm{R}$ to $\mathrm{P}$, we determine the number of pericyclic electrons that are transferred. These numbers, commonly referred to as yields, are evaluated as time integrals of the pericyclic electron fluxes from $t=0(\mathrm{R})$ to $t=\tau / 2(\mathrm{P})$. The corresponding time integral of the temporal factor $(\pi / \tau) \sin (2 \pi t / \tau)$ of $\left\langle j_{\mathrm{e}, \mathrm{NCM} \text {;peri,1D, } \varphi}(\varphi, t)\right\rangle_{\mathrm{bBOA}, \mathrm{R}}$ (see eqn (123)) is equal to $\pi / 4$. As a consequence, the yields are obtained by simply scaling the angular factors of $\left\langle j_{\mathrm{e}, \mathrm{NCM} \text {;peri,1D, } \varphi}(\varphi, t)\right\rangle_{\mathrm{bBOA}, \mathrm{R}}$ by the factor $\tau / 4$. The absolute values of the maximum numbers of pericyclic electrons transferred from $\mathrm{R}$ to $\mathrm{P}$ at the barrier positions of carbon nuclei $\mathrm{C} 2, \mathrm{C} 3, \mathrm{C} 4$, C6, C7, C8 are 0.63, 0.23, 0.63, 0.63, 0.23, 0.63, respectively (see Fig. 19b). These numbers are attached to the curved arrows in the Lewis structure of $\mathrm{R}$ in Fig. 19c. The maximum total 
Density

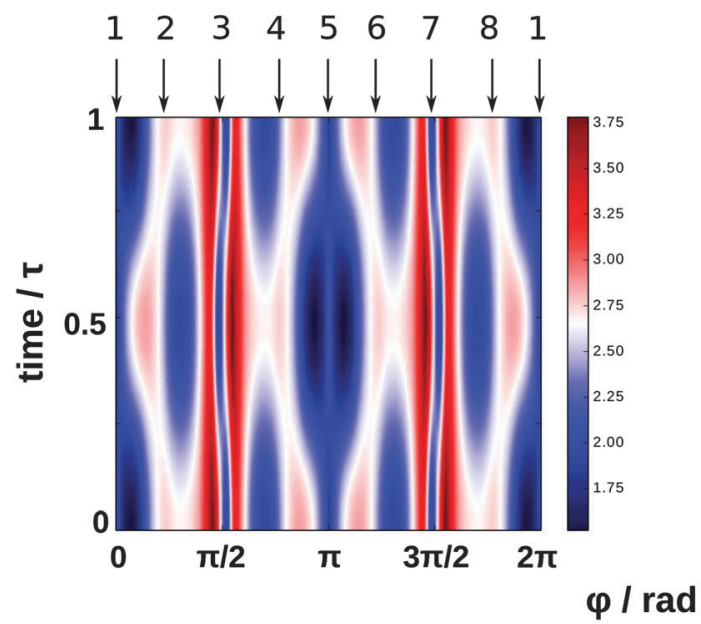

Flux Density

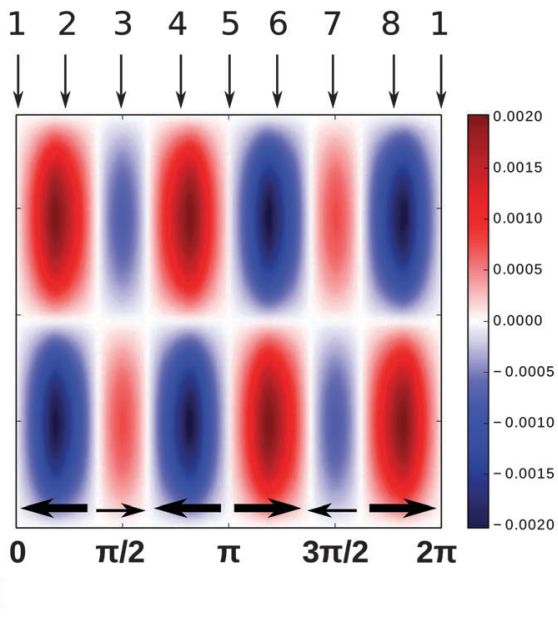

Fig. 20 Contour plots of time evolution of angular electronic probability densities (left) and flux densities (right) during Cope rearrangement of SBV in tunnelling domain. Vertical and horizontal arrows correspond to those shown in Fig. 19.

number of pericyclic electrons transferred during Cope rearrangement of SBV in the tunnelling domain is thus equal to 2.98. For comparison, we note that the number of pericyclic electrons transferred from one bond of $\mathrm{R}$ to the neighbouring bond of $\mathrm{P}$ through an observation plane that moves from $\mathrm{R}$ to $\mathrm{P}$, is of course smaller than 2.98; specifically it is equal to $1.1{ }^{27}$ In any case, the result of the present quantum computation of the number of pericyclic electrons transferred between neighbouring bonds during the model Cope rearrangement of SBV suggests that the textbook rules ${ }^{154-165}$ (i.e., cyclic series of curved arrows symbolizing the transfer of two electrons per curved arrow) need significant refinements. As we demonstrate, the number of transferred electrons that result from application of this rule in the present case of SBV, namely 6 , is far too large.

\section{Summary and conclusions}

In Section 2 we develop the quantum theory of concerted electronic and nuclear fluxes (CENFs) that accompany electronically adiabatic processes. Its applications in Sections 3-5 leads to important new insights into the nature of the CENFs associated with fundamental processes such as vibration and dissociation of single-electron diatomics (Section 3), as well as more complex processes such as chemical rearrangements of polyatomic inorganic (Section 4) and organic (Section 5) molecules.

A key development in Section 2 is the derivation of practical formulas for 1D flux densities (or fluxes) in specific directions. The derivation starts with the Schrödinger equation, whose formal solution yields a multi-dimensional continuity equation (CE). The multi-dimensional CE can then be reduced to a 1D CE that relates the rate of change of the probability density to the (1D) divergence of the corresponding flux density. The reduction is achieved by integration of the multi-dimensional CE over all coordinates, except the special one that describes the specific process of interest. The formula for the relevant 1D flux density (flux) is then obtained by integrating the rate of change of the (reduced) probability density over the special coordinate and invoking an appropriate boundary condition on the resulting 1D flux. This route to 1D fluxes was pioneered by Miller ${ }^{10,11}$ for applications to nuclear fluxes on the reaction coordinate of adiabatic reactions; here it is generalized for CENFs. The derivation employs mobile type coordinates that diagonalize the nuclear kinetic energy operator in the nuclear center of mass (NCM) frame. ${ }^{57-59}$ It invokes standard approximations (i.e., it neglects the effects of relativity and mass polarization and neglects the difference between the NCM and the total center of mass and between the electronic reduced mass and the electron mass). But it does not invoke the Born-Oppenheimer approximation (BOA), since all electronic fluxes vanish identically within the BOA. This approach, which reveals electronic fluxes "beyond BOA," is validated in Section 3, where it is applied to the prototype (aligned, vibrating $\mathrm{H}_{2}^{+}\left({ }^{2} \Sigma_{\mathrm{g}}^{+}\right)$), yielding CENFs in excellent agreement with benchmark results obtained by accurate, fully quantum B-spline spectral technique. ${ }^{50}$ We are aware of no other successful comparisons of independent numerical computations of CENFs analogous to the one described here. Section 3 also presents an encouraging comparison of 3D CENFs obtained by the scaled coupled-channels theory ${ }^{51}$ with accurate benchmark results ${ }^{50}$ again for the prototype.

A significant finding of Section 2 is the synchronicity of all CENFs associated with coherent tunneling between equivalent reactant (R) and product $(\mathrm{P})$ configurations of isolated molecules. A practical definition of the main directions of CENFs during coherent tunneling is also introduced in Section 2. It is found that the CENFs point from negative to positive domains of the difference of the densities of P and R. For NFDs, these directions are well approximated by the (vector) difference between classical equilibrium positions of the nuclei in $\mathrm{R}$ to $\mathrm{P}$. Yet another illuminating concept is the partitioning of EFDs 
into different categories corresponding to the character of the electrons (e.g., EFDs for core and valence electrons, corresponding to (reduced) densities of core and valence electrons). ${ }^{44}$ This partitioning relies on the possibility of expressing the total electron density approximately as a sum of (canonical) molecular orbital densities.

Specific new phenomena have been demonstrated for all model systems considered in Sections 3-5. Thus for the prototype we show the EFDs to be rather smooth compared with highly structured NFDs. This disparity is a consequence of the much lower mass of the electron compared with the nuclei, which leads to greater delocalization of the electrons compared with the nuclei. Nevertheless, even the EFDs may exhibit rich structure (e.g., multiple changes of the direction at a given instant). We might call this alternation of direction of the EFDs in space as the "electronic accordion effect" by analogy to the "nuclear accordion effect". 8 Furthermore, we can distinguish competing CENFs corresponding to vibration and dissociation, as well as interference between the two, in the case of isotropic $\mathrm{H}_{2}^{+}\left({ }^{2} \Sigma_{\mathrm{g}}{ }^{+}(J=0, M=0)\right)$ (the "quantum bubble").

For the relatively simple, but highly didactic, process in a model inorganic system, namely the isomerization of $\mathrm{B}_{4}$ in the tunneling domain, we demonstrate in Section 4 that core electrons flow with the nuclei. This confirms chemical intuition. In contrast, however, valence electrons may flow in oblique directions. We discover beautiful angular flux patterns of the valence electrons, with alternating a-c-a-c directions ("a" = clockwise, "c" = counterclockwise). Of course, all CENFs are synchronous, in accord with the general theorem derived in Section 2 .

In a more demanding treatment of coherent tunneling in a model organic system in Section 5, we compute the CENFs associated with the Cope rearrangement of semibullvalene (SBV), a so-called "peri-cyclic" reaction. The several phenomena discovered for $\mathrm{B}_{4}$ are also observed for SBV, suggesting the following extrapolation to tunnelling isomerizations in arbitrary systems: the synchronicity of all CENFs and the flow of core electrons in concert with the nuclei, in contrast with "pericyclic" electrons, which exhibit alternating clockwise and counterclockwise angular flux patterns, corresponding to "pincer-type" movement represented by a sequence of curved arrows in alternating directions in the Lewis structure of $\mathrm{R}$. This implies that "pericyclic" reactions are not really pericyclic. We find that the maximum yield of "pericyclic" electrons for the Cope rearrangement of SBV is a little less than 3, whereas the textbook rule would predict a transfer of 6 pericyclic electrons. ${ }^{154-165}$ The discrepancy can be traced back to incorrect assignments of two and one pericyclic electrons localized in double and single bonds of the traditional Lewis structure of R. In fact, the "pericyclic" electrons turn out to be delocalized beyond the double or single bonds (i.e., they penetrate into the domain of the neighboring bonds). As a consequence, they already partially occupy the region of the new bonds before flowing. It is not necessary for them to flow. Hence, the total number of electrons that actually flow is only approximately half of the numbers of transferred electrons estimated by the textbook rule.
The wealth of new discoveries documented in this perspective reflects the youth of the field of study of CENFs. It is easy to predict that future investigations will lead to exciting discoveries of rich, new phenomena associated with electronically adiabatic reactions in highly idealized model systems as well as practically important organic and inorganic systems. There is also plenty of room for additional fundamental and methodological development. Extensions to asymmetric systems (e.g., coherent tunneling in molecules exposed to a symmetrybreaking laser dipole interaction ${ }^{180}$ ) or to multi-dimensional models come easily to mind. A specific challenge is to observe the predicted CENFs experimentally. The recent deduction of the first experimental NFDs associated with vibrations of $\mathrm{Na}_{2}$ and $\mathrm{D}_{2}{ }^{+}$, derived from pump-probe spectroscopy, ${ }^{8,181,182}$ suggests possible analogous observations of EFDs. This is supported by recent developments of experimental techniques for the study of electron dynamics, ${ }^{183-186}$ in particular for specific categories of electrons that contribute to electron transfer. ${ }^{187}$ Work along these lines is in progress.

\section{Appendix A}

\section{A.1 Multi-dimensional continuity equation}

The transformed Hamiltonian (see eqn (24)) can be written

$$
H=-\frac{\hbar^{2}}{2 m_{\mathrm{e}}} \sum_{i=1}^{N_{\mathrm{e}}} \nabla_{\mathbf{q}_{i}}{ }^{2}-\sum_{\alpha=1}^{3 N_{\mathrm{n}}} \frac{\hbar^{2}}{2 \mu_{\alpha}} \frac{\partial^{2}}{\partial \tilde{Q}_{\alpha}{ }^{2}}+V(\mathbf{q}, \mathbf{Q})
$$

where the "squiggle" over $\tilde{Q}_{\alpha}$ indicates that the summation on $\alpha$ includes the NCM coordinates as well as the "internal" nuclear coordinates. The multi-dimensional Schrödinger equation is

$$
H \Psi(\mathbf{q}, \tilde{\mathbf{Q}}, t)=i \hbar \frac{\partial \Psi(\mathbf{q}, \tilde{\mathbf{Q}}, t)}{\partial t}
$$

where $\mathbf{q}$ and $\tilde{\mathbf{Q}}$ respectively stand for collections of electronic and nuclear coordinates. The probability density for observing the system to be in the configuration $(\mathbf{q}, \tilde{\mathbf{Q}})$ at time $t$ is

$$
\rho(\mathbf{q}, \tilde{\mathbf{Q}}, t)=|\Psi(\mathbf{q}, \tilde{\mathbf{Q}}, t)|^{2}
$$

We take $\Psi$ to be normalized. Differentiating eqn (A.3) with respect to time, we obtain

$$
\begin{aligned}
\frac{\partial \rho(\mathbf{q}, \tilde{\mathbf{Q}}, t)}{\partial t} & =\Psi^{*}(\mathbf{q}, \tilde{\mathbf{Q}}, t) \frac{\partial \Psi(\mathbf{q}, \tilde{\mathbf{Q}}, t)}{\partial t}+\Psi(\mathbf{q}, \tilde{\mathbf{Q}}, t) \frac{\partial \Psi^{*}(\mathbf{q}, \tilde{\mathbf{Q}}, t)}{\partial t} \\
& =-\frac{i}{\hbar}\left[\Psi^{*} H \Psi-\Psi H \Psi^{*}\right]
\end{aligned}
$$

where the second line follows from eqn (A.2). Substitution of the explicit expression for $H$ (eqn (A.1)) into eqn (A.4) yields

$$
\begin{aligned}
\frac{\partial \rho}{\partial t}= & -\sum_{i=1}^{N_{\mathrm{e}}} \frac{\hbar}{2 i m_{\mathrm{e}}}\left[\Psi^{*} \nabla_{\mathbf{q}_{i}}{ }^{2} \Psi-\Psi \nabla_{\mathbf{q}_{i}}{ }^{2} \Psi^{*}\right] \\
& -\sum_{\alpha=1}^{3 N_{\mathrm{n}}} \frac{\hbar}{2 i \mu_{\alpha}}\left[\Psi^{*} \partial^{2} \Psi / \partial \tilde{Q}_{\alpha}{ }^{2}-\Psi \partial^{2} \Psi^{*} / \partial \tilde{Q}_{\alpha}{ }^{2}\right]
\end{aligned}
$$


where the contributions from the potential energy cancel each other. Eqn (A.5) can be rewritten

$$
\frac{\partial \rho}{\partial t}+\sum_{i=1}^{N_{\mathrm{e}}} \nabla_{\mathbf{q}_{i}} \cdot \mathbf{J}_{i}+\sum_{\alpha=1}^{3 N_{\mathrm{n}}} \frac{\partial}{\partial \tilde{Q}_{\alpha}} J_{\alpha}=0
$$

where

$$
\begin{gathered}
\mathbf{J}_{i}(\mathbf{q}, \tilde{\mathbf{Q}}, t) \equiv \frac{\hbar}{2 i m_{\mathrm{e}}}\left[\Psi^{*} \nabla_{\mathbf{q}_{i}} \Psi-\Psi \nabla_{\mathbf{q}_{i}} \Psi^{*}\right] \\
J_{\alpha}(\mathbf{q}, \tilde{\mathbf{Q}}, t) \equiv \frac{\hbar}{2 i \mu_{\alpha}}\left[\Psi^{*} \partial \Psi / \partial \tilde{Q}_{\alpha}-\Psi \partial \Psi^{*} / \partial \tilde{Q}_{\alpha}\right]
\end{gathered}
$$

Eqn (A.6) is the multi-dimensional continuity equation (CE). ${ }^{2}$ The quantities defined in eqn (A.7) can be regarded, respectively, as electron and nuclear flux densities in the $3 N \mathrm{D}$ space (where $N=N_{\mathrm{e}}+N_{\mathrm{n}}$ ) of particle coordinates. All flux densities have dimensions $1 / L^{3 N-1} T$.

\section{A.2 Reduction of the multi-dimensional continuity equation}

Integrating eqn (A.6) over all nuclear coordinates and all electronic coordinates except those of the "first" electron, we obtain

$$
\begin{array}{r}
\int \mathrm{d} \tilde{\mathbf{Q}} \prod_{j \neq 1} \int \mathrm{d} \mathbf{q}_{j} \partial \rho(\mathbf{q}, \tilde{\mathbf{Q}}, t) / \partial t+\sum_{k \neq 1} \int \mathrm{d} \mathbf{q}_{k} \nabla_{\mathbf{q}_{k}} \\
\cdot \int \mathrm{d} \tilde{\mathbf{Q}} \prod_{j \neq k \neq 1} \int \mathrm{d} \mathbf{q}_{j} \mathbf{J}_{k}+\nabla_{\mathbf{q}_{1}} \cdot \int \mathrm{d} \tilde{\mathbf{Q}} \prod_{j \neq 1} \int \mathrm{d} \mathbf{q}_{j} \mathbf{J}_{1} \\
+\sum_{\alpha} \int \mathrm{d} \tilde{\mathbf{Q}}_{\alpha} \frac{\partial}{\partial \tilde{Q}_{\alpha}} \prod_{\beta \neq \alpha} \int \mathrm{d} \tilde{\mathbf{Q}}_{\beta} \prod_{j \neq 1} \int \mathrm{d} \mathbf{q}_{j} J_{\alpha}=0
\end{array}
$$

The second and fourth terms of eqn (A.8) vanish. We can recast the second term as

$$
\begin{aligned}
\sum_{k \neq 1} \int \mathrm{d} \mathbf{q}_{k} \nabla_{\mathbf{q}_{k}} \cdot \int \mathrm{d} \tilde{\mathbf{Q}} \prod_{j \neq k \neq 1} \int \mathrm{d} \mathbf{q}_{j} \mathbf{J}_{k} & =\int \mathrm{d} \mathbf{q}_{k} \nabla_{\mathbf{q}_{k}} \cdot \mathbf{f}_{1}\left(\mathbf{q}_{1}, \mathbf{q}_{k}, t\right) \\
& =\int_{S} \mathrm{~d} S \mathbf{n} \cdot \mathbf{f}_{1}\left(\mathbf{q}_{1}, \mathbf{q}_{k}, t\right)
\end{aligned}
$$

where the vector function $\mathbf{f}_{1}$ is defined implicitly and we exploit the divergence theorem to get the second line, regarding the vector $\mathbf{q}_{1}$ as a parameter. The surface $S$ must be at an infinite remove, as the integration on $\mathbf{q}_{k}$ runs over an infinite volume. Since $\Psi$ is normalized, the surface integral must disappear. In a similar way the fourth term disappears according to the following:

$$
\begin{aligned}
& \sum_{\alpha} \int \mathrm{d} \tilde{Q}_{\alpha} \frac{\partial}{\partial \tilde{Q}_{\alpha}} \prod_{\beta \neq \alpha} \int \mathrm{d} \tilde{Q}_{\beta} \prod_{j \neq 1} \int \mathrm{d} \mathbf{q}_{j} J_{\alpha} \\
& \quad=\sum_{\alpha} \int \mathrm{d} \tilde{Q}_{\alpha} \frac{\partial}{\partial \tilde{Q}_{\alpha}} F_{\alpha}\left(\mathbf{q}_{1}, Q_{\alpha}\right) \\
& \quad=\sum_{\alpha} F_{\alpha}\left(\mathbf{q}_{1}, Q_{\alpha}=\infty\right)-F_{\alpha}\left(\mathbf{q}_{1}, Q_{\alpha}=-\infty\right)=0
\end{aligned}
$$

where the ancillary function $F_{\alpha}$ is defined implicitly. The continuity equation (eqn (A.6)) then reduces to

$$
\frac{\partial\left\langle\rho_{1}\left(\mathbf{q}_{1}, t\right)\right\rangle}{\partial t}+\nabla_{\mathbf{q}_{1}} \cdot\left\langle\mathbf{j}_{1}\left(\mathbf{q}_{1}, t\right)\right\rangle=0
$$

where

$$
\begin{gathered}
\left\langle\rho_{1}\left(\mathbf{q}_{1}, t\right)\right\rangle \equiv \int \mathrm{d} \tilde{\mathbf{Q}} \prod_{j \neq 1} \int \mathrm{d} \mathbf{q}_{j} \rho(\mathbf{q}, \tilde{\mathbf{Q}}, t) \\
\left\langle\mathbf{j}_{1}\left(\mathbf{q}_{1}, t\right)\right\rangle \equiv \frac{\hbar}{2 i m_{\mathrm{e}}} \int \mathrm{d} \tilde{\mathbf{Q}} \prod_{j \neq 1} \int \mathrm{d} \mathbf{q}_{j}\left[\Psi^{*} \nabla_{\mathbf{q}_{1}} \Psi-\Psi \nabla_{\mathbf{q}_{1}} \Psi^{*}\right]
\end{gathered}
$$

are, respectively, the probability density of observing electron 1 in volume element $d \mathbf{q}_{1}$ about $\mathbf{q}_{1}$ and the flux density of electron 1 at point of observation $\mathbf{q}_{1}$. We can derive the analogue of eqn (A.11) for the other electrons, so that for any particular electron $i$ we can write

$$
\frac{\partial\left\langle\rho_{i}(\mathbf{x}, t)\right\rangle}{\partial t}+\nabla_{\mathbf{x}} \cdot\left\langle\mathbf{j}_{i}(\mathbf{x}, t)\right\rangle=0
$$

where

$$
\begin{gathered}
\left.\left\langle\rho_{i}(\mathbf{x}, t)\right\rangle \equiv \int \mathrm{d} \tilde{\mathbf{Q}} \prod_{j \neq i} \int \mathrm{d} \mathbf{q}_{j} \rho(\mathbf{q}, \tilde{\mathbf{Q}}, t)\right|_{\mathbf{q}_{i}=\mathbf{x}} \\
\left.\left\langle\mathbf{j}_{i}(\mathbf{x}, t)\right\rangle \equiv \frac{\hbar}{2 i m_{\mathrm{e}}} \int \mathrm{d} \tilde{\mathbf{Q}} \prod_{j \neq i} \int \mathrm{d} \mathbf{q}_{j}\left[\Psi^{*} \nabla_{\mathbf{q}_{i}} \Psi-\Psi \nabla_{\mathbf{q}_{i}} \Psi^{*}\right]\right|_{\mathbf{q}_{i}=\mathbf{x}}
\end{gathered}
$$

where $\mathbf{x}$ denotes a point in 3D space. Summing eqn (A.13) over all electrons and invoking the equivalence of electrons, we get eqn (31a).

Integration of the multi-dimensional CE (eqn (A.6)) over all electronic coordinates produces

$$
\int \mathrm{d} \mathbf{q} \partial \rho(\mathbf{q}, \tilde{\mathbf{Q}}, t) / \partial t+\sum_{k} \int \mathrm{d} \mathbf{q}_{k} \nabla_{\mathbf{q}_{k}} \cdot \prod_{j \neq k} \int \mathrm{d} \mathbf{q}_{j} \mathbf{J}_{k}+\sum_{\alpha} \frac{\partial}{\partial \tilde{Q}_{\alpha}} \int \mathrm{d} \mathbf{q} J_{\alpha}=0
$$

Again appealing to the divergence theorem and the normalization of the wave function, we deduce that the second term of eqn (A.15) is zero. Thus, we obtain a reduced CE for the nuclear coordinates:

$$
\partial\left\langle\rho_{\mathrm{n}}(\tilde{\mathbf{Q}}, t)\right\rangle / \partial t+\sum_{\alpha} \frac{\partial}{\partial \tilde{Q}_{\alpha}}\left\langle J_{\mathrm{n} \alpha}(\tilde{\mathbf{Q}}, t)\right\rangle=0
$$

where we define

$$
\begin{aligned}
\left\langle\rho_{\mathrm{n}}(\tilde{\mathbf{Q}}, t)\right\rangle & \equiv \int \mathrm{d} \mathbf{q} \rho(\mathbf{q}, \tilde{\mathbf{Q}}, t) \\
\left\langle J_{\mathrm{n} \alpha}(\tilde{\mathbf{Q}}, t)\right\rangle & \equiv \int \mathrm{d} \mathbf{q} J_{\alpha}(\mathbf{q}, \tilde{\mathbf{Q}}, t)
\end{aligned}
$$


By integrating eqn (A.16) over all except one of the nuclear coordinates, say $Q_{\alpha}$, we get

$$
\begin{aligned}
& \partial\left[\prod_{\beta \neq \alpha} \int \mathrm{d} Q_{\beta}\left\langle\rho_{\mathrm{n}}(\tilde{\mathbf{Q}}, t)\right\rangle\right] / \partial t \\
& \quad+\sum_{\beta^{\prime} \neq \alpha} \int \mathrm{d} \tilde{Q}_{\beta^{\prime}} \frac{\partial}{\partial \tilde{Q}_{\beta^{\prime}}}\left\{\prod_{\beta \neq \alpha \neq \beta^{\prime}} \int \mathrm{d} \tilde{Q}_{\beta}\left\langle J_{\mathrm{n} \beta^{\prime}}(\tilde{\mathbf{Q}}, t)\right\rangle\right\} \\
& \quad+\frac{\partial}{\partial \tilde{Q}_{\alpha}}\left[\prod_{\beta \neq \alpha} \int d \tilde{Q}_{\beta}\left\langle J_{\mathrm{n} \alpha}(\tilde{\mathbf{Q}}, t)\right\rangle\right]=0
\end{aligned}
$$

The second term of eqn (A.18) disappears as a consequence of the same reasoning applied to the fourth term of eqn (A.8). Hence, eqn (A.18) reduces to the $1 \mathrm{D} \mathrm{CE}$

$$
\partial\left\langle\rho_{\alpha}\left(\tilde{Q}_{\alpha}, t\right)\right\rangle / \partial t+\frac{\partial\left\langle j_{\alpha}\left(\tilde{Q}_{\alpha}, t\right)\right\rangle}{\partial \tilde{Q}_{\alpha}}=0
$$

Note that the dimensions of the terms are $1 / L T$. For the $1 \mathrm{D}$ model (see Section 2.6) eqn (A.19) is equivalent to eqn (44b).

By manipulations paralleling those employed above we can derive the version of the $3 \mathrm{ND} \mathrm{CE}$ in the laboratory coordinates:

$$
\frac{\partial \rho}{\partial t}+\sum_{i=1}^{N_{\mathrm{e}}} \nabla_{q_{i}} \cdot \mathbf{J}_{i}+\sum_{a=1}^{N_{\mathrm{n}}} \sum_{\gamma=x, y, z} \frac{\partial}{\partial R_{a \gamma}} J_{a \gamma}=0
$$

here $\gamma$ labels Cartesian components of the nuclear coordinates and the $3 N D$ nuclear flux density is given by

$$
J_{a \gamma}(\mathbf{r}, \mathbf{R}, t) \equiv \frac{\hbar}{2 i M_{\mathrm{a}}}\left[\Psi^{*} \partial \Psi / \partial R_{a \gamma}-\Psi \partial \Psi^{*} / \partial R_{a \gamma}\right]
$$

\section{A.3 Relation between nuclear flux densities in the laboratory and internal frames}

To compute the flux density associated with the Cartesian component $R_{a \gamma}$ of nucleus $a$ at point of observation $R$ (i.e., the flux of nucleus $a$ in the $\gamma$-direction at point of observation having the Cartesian component $R_{a \gamma}=R$ ), we multiply eqn (A.21) by the Dirac distribution $\delta\left(R-R_{a \gamma}\right)$ and integrate over all laboratory coordinates. This procedure gives

$$
\begin{aligned}
\left\langle j_{a \gamma}(R, t)\right\rangle= & \int \mathrm{d} \mathbf{r} \int \mathrm{d} \mathbf{R} \delta\left(R-R_{a \gamma}\right) J_{a \gamma}(\mathbf{r}, \mathbf{R}, t) \\
= & \frac{\hbar}{2 i M_{a}} \int \mathrm{d} \mathbf{r} \prod_{b \neq a} \prod_{\lambda} \int \mathrm{d} R_{b \lambda} \prod_{\lambda^{\prime}} \int \mathrm{d} R_{a \lambda^{\prime}} \delta\left(R-R_{a \gamma}\right) \\
& \times\left[\Psi^{*} \partial \Psi / \partial R_{a \gamma}-\Psi \partial \Psi^{*} / \partial R_{a \gamma}\right] \\
= & \frac{\hbar}{2 i M_{a}} \int \mathrm{d} \mathbf{r} \prod_{b \neq a} \prod_{\lambda} \int \mathrm{d} R_{b \lambda} \prod_{\lambda^{\prime} \neq \gamma} \int \mathrm{d} R_{a \lambda^{\prime}} \\
& \times\left.\left[\Psi^{*} \partial \Psi / \partial R_{a \gamma}-\Psi \partial \Psi^{*} / \partial R_{a \gamma}\right]\right|_{R_{a \gamma}=R}
\end{aligned}
$$

We now transform from laboratory to internal nuclear (plus NCM) coordinates. From eqn (18) and (19), we have

$$
\begin{aligned}
R_{a \gamma} & =\sum_{\alpha}\left[\tilde{\mathbf{W}}^{-1}\right]_{a \gamma ; \alpha} \tilde{Q}_{\alpha} \\
\frac{\partial}{\partial R_{a \gamma}} & =\sum_{\alpha}\left[\tilde{\mathbf{W}}^{\mathrm{T}}\right]_{a \gamma ; \alpha} \frac{\partial}{\partial \tilde{Q}_{\alpha}}
\end{aligned}
$$

Substituting these relations into the second line of eqn (A.22), we get

$$
\begin{aligned}
\left\langle j_{a \gamma}(R, t)\right\rangle= & \frac{\hbar}{2 i M_{a}} \int \mathrm{d} \mathbf{q} \int \mathrm{d} \tilde{\mathbf{Q}} \delta\left[R-\sum_{\beta}\left[\tilde{\mathbf{W}}^{-1}\right]_{a \gamma ; \beta} \tilde{Q}_{\beta}\right] \\
& \times \sum_{\alpha}\left[\tilde{\mathbf{W}}^{\mathrm{T}}\right]_{a \gamma ; \alpha}\left[\Psi^{*} \frac{\partial \Psi}{\partial \tilde{Q}_{\alpha}}-\Psi \frac{\partial \Psi^{*}}{\partial \tilde{Q}_{\alpha}}\right] \\
= & \frac{1}{M_{a}} \int \mathrm{d} \mathbf{q} \int \mathrm{d} \tilde{\mathbf{Q}} \delta\left[R-\sum_{\beta}\left[\tilde{\mathbf{W}}^{-1}\right]_{a \gamma ; \beta} \tilde{Q}_{\beta}\right] \\
& \times \sum_{\alpha}\left[\tilde{\mathbf{W}}^{\mathrm{T}}\right]_{a \gamma ; \alpha} \mu_{\alpha} \frac{\hbar}{2 i \mu_{\alpha}}\left[\Psi^{*} \frac{\partial \Psi}{\partial \tilde{Q}_{\alpha}}-\Psi \frac{\partial \Psi^{*}}{\partial \tilde{Q}_{\alpha}}\right]
\end{aligned}
$$

where we note that the Jacobian is $|\operatorname{det}(\tilde{\mathbf{W}})|^{-1}=1$. We next integrate over $\tilde{Q}_{\alpha}$. For this purpose, we recast the Dirac distribution as

$$
\begin{aligned}
& \delta\left[R-\sum_{\beta}\left[\tilde{\mathbf{W}}^{-1}\right]_{a \gamma ; \beta} \tilde{Q}_{\beta}\right] \\
& \quad=\delta\left\{-\left(\tilde{\mathbf{W}}^{-1}\right)_{a \gamma ; \alpha}\left[\tilde{Q}_{\alpha}-\frac{R}{\left(\tilde{\mathbf{W}}^{-1}\right)_{a \gamma ; \alpha}}+\sum_{\beta \neq \alpha} \frac{\left(\tilde{\mathbf{W}}^{-1}\right)_{a \gamma ; \beta}}{\left(\tilde{\mathbf{W}}^{-1}\right)_{a \gamma ; \alpha}} \tilde{Q}_{\beta}\right]\right\} \\
& =\frac{1}{\left|\left(\tilde{\mathbf{W}}^{-1}\right)_{a \gamma ; \alpha}\right|} \delta\left\{\tilde{Q}_{\alpha}-\left[\frac{R}{\left(\tilde{\mathbf{W}}^{-1}\right)_{a \gamma ; \alpha}}-\sum_{\beta \neq \alpha} \frac{\left(\tilde{\mathbf{W}}^{-1}\right)_{a \gamma ; \beta}}{\left(\tilde{\mathbf{W}}^{-1}\right)_{a \gamma ; \alpha}} \tilde{Q}_{\beta}\right]\right\}
\end{aligned}
$$

where the second line relies on the identity $\delta(a x)=|a|^{-1} \delta(x)$. Plugging eqn (A.25) into eqn (A.24) and integrating on $Q_{\alpha}$ yields

$$
\begin{aligned}
& \left\langle j_{a \gamma}(R, t)\right\rangle=\frac{1}{M_{a}} \sum_{\alpha} \frac{\left[\tilde{\mathbf{W}}^{\mathrm{T}}\right]_{a \gamma ; \alpha}}{\left|\left[\tilde{\mathbf{W}}^{-1}\right]_{a \gamma ; \alpha}\right|} \mu_{\alpha} \frac{\hbar}{2 i \mu_{\alpha}} \int \mathrm{d} \mathbf{q} \prod_{\beta \neq \alpha} \int \mathrm{d} \tilde{Q}_{\beta} \\
& \times\left[\Psi^{*} \frac{\partial \Psi}{\partial \tilde{Q}_{\alpha}}-\Psi \frac{\partial \Psi^{*}}{\partial \tilde{Q}_{\alpha}}\right] \mid \tilde{Q}_{\alpha=R} /\left(\tilde{\mathbf{w}}^{-1}\right)_{a \gamma ; \alpha}-\sum_{\beta \neq \alpha}\left(\tilde{\mathbf{w}}^{-1}\right)_{a \gamma ; \beta} \tilde{Q}_{\beta} /\left(\tilde{\mathbf{w}}^{-1}\right)_{a \gamma ; \alpha}
\end{aligned}
$$

For the 1D model, which involves only a single special coordinate, eqn (A.26) reduces to

$$
\left\langle j_{a \gamma}(R, t)\right\rangle=\frac{\mu_{\alpha}\left[\tilde{\mathbf{W}}^{\mathrm{T}}\right]_{a \gamma ; \alpha}}{M_{a}\left|\left[\tilde{\mathbf{W}}^{\mathrm{T}}\right]_{a \gamma ; \alpha}\right|}\left\langle j_{\alpha}\left(R /\left[\tilde{\mathbf{W}}^{-1}\right]_{a \gamma ; \alpha}, t\right)\right\rangle
$$

in accord with eqn (51).

A possible alternate route to the relation between laboratory and internal nuclear fluxes is the following. From eqn (A.23b) 
we have

$$
\frac{\hbar}{i M_{a}} \Psi^{*} \frac{\partial \Psi}{\partial R_{a \gamma}}=\frac{1}{M_{a}} \sum_{\alpha}\left[\tilde{\mathbf{W}}^{\mathrm{T}}\right]_{a \gamma ; \alpha} \mu_{\alpha} \frac{\hbar}{i \mu_{\alpha}} \Psi^{*} \frac{\partial \Psi}{\partial Q_{\alpha}}
$$

where we pre- and post-multiply by $\Psi^{*}$ and $\Psi$, respectively. Adding eqn (A.28) to its complex conjugate yields

$$
\begin{aligned}
& \frac{\hbar}{2 i M_{a}}\left[\Psi^{*} \frac{\partial \Psi}{\partial R_{a \gamma}}-\Psi \frac{\partial \Psi^{*}}{\partial R_{a \gamma}}\right] \\
& =\frac{1}{M_{a}} \sum_{\alpha}\left[\tilde{\mathbf{W}}^{\mathrm{T}}\right]_{a \gamma ; \alpha} \mu_{\alpha} \frac{\hbar}{2 i \mu_{\alpha}}\left[\Psi^{*} \frac{\partial \Psi}{\partial \tilde{Q}_{\alpha}}-\Psi \frac{\partial \Psi^{*}}{\partial \tilde{Q}_{\alpha}}\right]
\end{aligned}
$$

Using the definitions in eqn (A.7b) and (A.21), we can cast eqn (A.29) as

$$
J_{a \gamma}(\mathbf{r}, \mathbf{R}, t)=\frac{1}{M_{a}} \sum_{\alpha}\left[\tilde{\mathbf{W}}^{\mathrm{T}}\right]_{a \gamma ; \alpha} \mu_{\alpha} J_{\alpha}(\mathbf{q}, \tilde{\mathbf{Q}}, t)
$$

or, in matrix form as

$$
\mathbf{J}_{\mathrm{L}}=\mathbf{M}^{-1} \tilde{\mathbf{W}}^{\mathrm{T}} \boldsymbol{\mu} \mathbf{J}_{\mathrm{I}}
$$

where the subscripts I and $\mathrm{L}$ are intended to distinguish between "internal" and "laboratory."

Incidentally, one could take eqn (A.30) as the starting point for the derivation of eqn (A.26). That is, one could multiply both sides of eqn (A.30) by $\delta\left(R-R_{a \gamma}\right)$ and integrate the resulting equation over all electronic and nuclear coordinates.

\section{Appendix B}

Our purpose here is to provide a brief derivation of eqn (101). We begin by observing in eqn (100) that the mobile-type coordinates $\left\{Q_{2}, Q_{7}, Q_{8}, S_{z}\right\}$ depend only on the $z$-components of the laboratory Cartesian coordinates of the atoms; the remaining mobile-type coordinates $\left\{Q_{1}, Q_{3}, Q_{4}, Q_{5}, Q_{6}, Q_{9}, S_{x}\right.$, $\left.S_{y}\right\}$ depend only the $x$ - and $y$-components of the atomic coordinates. As a consequence the rows of eqn (100) may be reordered so that $\tilde{\mathbf{W}}$ assumes block-diagonal form: a $4 \times 4$ block corresponding to the first subset of mobile-types and an $8 \times 8$ corresponding to the second subset.

To demonstrate the procedure, we consider the set of four equations, which can be written explicitly as

$$
\left(\begin{array}{c}
Q_{2} \\
Q_{7} \\
Q_{8} \\
S_{z}
\end{array}\right)=\left(\begin{array}{cccc}
1 / 2 & -1 / 2 & 1 / 2 & -1 / 2 \\
0 & 1 & 0 & -1 \\
-1 & 0 & 1 & 0 \\
1 / 4 & 1 / 4 & 1 / 4 & 1 / 4
\end{array}\right)\left(\begin{array}{c}
Z_{1} \\
Z_{2} \\
Z_{3} \\
Z_{4}
\end{array}\right)
$$

The determinant of the reformed $4 \times 4$ sub-matrix is unity. From eqn (20) and (B.1) we have the following relation between the components of momentum conjugate to laboratory and mobile coordinates:

$$
\left(\begin{array}{c}
P_{1 z} \\
P_{2 z} \\
P_{3 z} \\
P_{4 z}
\end{array}\right)=\left(\begin{array}{cccc}
1 / 2 & 0 & -1 & 1 / 4 \\
-1 / 2 & 1 & 0 & 1 / 4 \\
1 / 2 & 0 & 1 & 1 / 4 \\
-1 / 2 & -1 & 0 & 1 / 4
\end{array}\right)\left(\begin{array}{c}
P_{2} \\
P_{7} \\
P_{8} \\
P_{S_{z}}
\end{array}\right)
$$

where we note that the matrix in eqn (B.2) is just the transpose of that in eqn (B.1). The contribution to the nuclear kinetic energy of the four $z$-components of the laboratory momenta can then be written in terms of the mobile momenta as

$$
\begin{aligned}
T_{4} \equiv & (2 M)^{-1}\left[P_{1 z}{ }^{2}+P_{2 z}{ }^{2}+P_{3 z}{ }^{2}+P_{4 z}{ }^{2}\right]=(2 M)^{-1}\left[\left(P_{2} / 2-P_{8}+P_{S_{z}} / 4\right)^{2}\right. \\
& +\left(-P_{2} / 2+P_{7}+P_{S_{z}} / 4\right)^{2}+\left(P_{2} / 2+P_{8}+P_{S_{z}} / 4\right)^{2} \\
& \left.+\left(-P_{2} / 2-P_{7}+P_{S_{z}} / 4\right)^{2}\right]
\end{aligned}
$$

where the coefficients of the mobile components for a given laboratory $z$-component are just the elements of the corresponding row of the matrix (see eqn (B.2)). It is straightforward, but arduous, to show that the cross terms in $T_{4}$ vanish, leaving the diagonal contribution

$$
T_{4} \equiv P_{2}^{2} / 2\left(M_{\mathrm{B}}\right)+P_{7}^{2} / 2\left(M_{\mathrm{B}} / 2\right)+P_{8}^{2} / 2\left(M_{\mathrm{B}} / 2\right)+P_{S_{z}}{ }^{2} / 2\left(4 M_{\mathrm{B}}\right)
$$

Applying the same procedure to the second set of eight equations, we obtain

$$
\begin{aligned}
T_{8} \equiv & P_{1}^{2} / 2\left(M_{\mathrm{B}} / 4\right)+P_{3}{ }^{2} / 2\left(M_{\mathrm{B}}\right)+P_{4}{ }^{2} / 2\left(M_{\mathrm{B}}\right)+P_{5}{ }^{2} / 2\left(M_{\mathrm{B}}\right) \\
& +P_{6}{ }^{2} / 2\left(M_{\mathrm{B}} / 4\right)+P_{9}{ }^{2} / 2\left(M_{\mathrm{B}}\right)+{P_{S_{x}}}^{2} / 2\left(4 M_{\mathrm{B}}\right)+P_{S_{y}}{ }^{2} / 2\left(4 M_{\mathrm{B}}\right)
\end{aligned}
$$

Eqn (B.4) and (B.5) are so written as to indicate the reduced masses associated with the mobile coordinates, which are: $\mu_{1}=$ $\mu_{6}=M_{\mathrm{B}} / 4 ; \mu_{2}=\mu_{3}=\mu_{4}=\mu_{5}=\mu_{9}=M_{\mathrm{B}} ; \mu_{7}=\mu_{8}=M_{\mathrm{B}} / 2 ; \mu_{S_{x}}=\mu_{S_{y}}=$ $\mu_{S_{z}}=4 M_{\mathrm{B}}$. The sum $T_{4}+T_{8}$ is equal to $T_{\mathrm{n}}$ given by eqn (101).

It is instructive to derive expressions for the reduced masses of the mobile coordinates via the intuitive approach described in the text just below eqn (22) or (98). For example, we consider $Q_{2}$, the out-of-plane bending vibration. According to the schematic diagram in Fig. 11, atoms 1 and 3 move in the positive $z$-direction as atoms 2 and 4 move in the negative $z$-direction. We regard the 1-3 and 2-4 diatomic pairs as pseudo-atoms. The motion of their centers of mass with respect to each other along the $z$-axis constitutes a vibration, whose associated reduced mass is $\mu_{2}=2 M_{\mathrm{B}} \times 2 M_{\mathrm{B}} /\left(2 M_{\mathrm{B}}+2 M_{\mathrm{B}}\right)=M_{\mathrm{B}}$, which agrees with the analytic result obtained above. The diagram for $Q_{7}$ (see Fig. 11) depicts rotation about a line (i.e., the $x$-axis) passing through atoms 1 and 3 . The atoms 2 and 4 constitute a pseudo-diatomic rotating in the $x-y$ plane. The associated moment of inertia is $I=\mu_{7} R^{2}=M_{\mathrm{B}}\left[(R / 2)^{2}+(R / 2)^{2}\right]$, where each of the atoms of the diatomic is a distance $R / 2$ removed from the axis of rotation. We conclude that $\mu_{7}=M_{\mathrm{B}} / 2$, which is in accord with the analytic result. A similar argument can be applied to $Q_{8}$, which is rotation about the $y$-axis. The translation of the whole molecule in the $z$-direction is described by $S_{z}$. The associated reduced mass must therefore be the mass of the whole molecule, $\mu_{S_{z}}=4 M_{\mathrm{B}}$. 
Similar rationales can be used to derive the reduced masses of the modes in the second set of mobile-types.

\section{Appendix C}

According to eqn (51b),

$$
\left\langle j_{a \gamma}\left(R_{a \gamma}, t\right)\right\rangle=\frac{\mu_{\alpha}}{M_{a}} \frac{\left[\tilde{\mathbf{W}}^{\mathrm{T}}\right]_{a \gamma ; \alpha}}{\left|\left[\tilde{\mathbf{W}}^{-1}\right]_{a \gamma ; \alpha}\right|}\left\langle j_{\alpha}\left(Q_{\alpha}, t\right)\right\rangle
$$

the fluxes of the individual nuclei $a$ along Cartesian coordinates $R_{a \gamma}$ are proportional to the fluxes associated with the mobiletype coordinate $\alpha$. Our purpose here is to show that, for the particular case of $\mathrm{B}_{4}$ treated in Section 4 , the constant of proportionality is given by

$$
c=\frac{\mu_{\alpha}}{M_{\mathrm{B}}} \frac{\left[\tilde{\mathbf{W}}^{\mathrm{T}}\right]_{a \gamma ; \alpha}}{\left|\left[\tilde{\mathbf{W}}^{-1}\right]_{a \gamma ; \alpha}\right|}=\operatorname{sign}\left(\left[\tilde{\mathbf{W}}^{\mathrm{T}}\right]_{a \gamma ; \alpha}\right)
$$

where we have set $M_{a}=M_{\mathrm{B}}$ for all atoms.

We start with the observation that the rows of $\tilde{\mathbf{W}}$ are orthogonal, although they are not normalized (see eqn (100)). This condition can be expressed by

$$
\begin{gathered}
\sum_{k}[\tilde{\mathbf{W}}]_{i k}[\tilde{\mathbf{W}}]_{j k}=0, \quad i \neq j \\
\sum_{k}[\tilde{\mathbf{W}}]_{i k}[\tilde{\mathbf{W}}]_{i k}=a_{i}^{2}, \quad i=j
\end{gathered}
$$

where we condense the notation temporarily, letting $i$ and $j$ stand for $a \gamma$ and $k$ for $\alpha$. Eqn (C.3) can be rewritten succinctly as

$$
\sum_{k}[\tilde{\mathbf{W}}]_{i k}[\tilde{\mathbf{W}}]_{j k} / a_{j}^{2}=\sum_{k}[\tilde{\mathbf{W}}]_{i k}\left[\tilde{\mathbf{W}}^{\mathrm{T}}\right]_{k j} / a_{j}^{2}=\delta_{i j}
$$

from which it follows that

$$
\left[\tilde{\mathbf{W}}^{-1}\right]_{k j}=\left[\tilde{\mathbf{W}}^{\mathrm{T}}\right]_{k j} / a_{j}^{2}=\frac{\left[\tilde{\mathbf{W}}^{\mathrm{T}}\right]_{k j}}{\sum_{l}[\tilde{\mathbf{W}}]_{j l}^{2}}
$$

We now insert this latter expression for the inverse into that for $c$ in eqn (C.2), obtaining

$$
c=\frac{\mu_{\alpha}}{M_{\mathrm{B}}} \frac{\left[\tilde{\mathbf{W}}^{\mathrm{T}}\right]_{a \gamma ; \alpha}}{\left|\left[\tilde{\mathbf{W}}^{\mathrm{T}}\right]_{a \gamma ; \alpha}\right|} \sum_{a^{\prime} \gamma^{\prime}} \tilde{\mathbf{W}}_{\alpha ; a^{\prime} \gamma^{\prime}}{ }^{2}=\frac{\mu_{\alpha}}{M_{\mathrm{B}}} \operatorname{sign}\left(\left[\tilde{\mathbf{W}}^{\mathrm{T}}\right]_{a \gamma ; \alpha}\right) \sum_{a^{\prime} \gamma^{\prime}}[\tilde{\mathbf{W}}]_{\alpha ; a^{\prime} \gamma^{\prime}}{ }^{2}
$$

From eqn (20) we have, in the present notation, the relation between the momenta conjugate to the Cartesian laboratory components and the momenta conjugate to the mobile-type coordinates:

$$
P_{a \gamma}=\sum_{\alpha}\left[\mathbf{W}^{\mathrm{T}}\right]_{a \gamma ; \alpha} P_{\alpha}
$$

Hence, we can write the total nuclear kinetic energy as

$$
\begin{aligned}
T_{\mathrm{n}} & =\sum_{a \gamma} P_{a \gamma}{ }^{2} / 2 M_{\mathrm{B}}=\sum_{a \gamma}\left[\sum_{\alpha}\left[\mathbf{W}^{\mathrm{T}}\right]_{a \gamma ; \alpha} P_{\alpha}\right]^{2} / 2 M_{\mathrm{B}} \\
& =\sum_{\alpha} P_{\alpha}{ }^{2} / 2 \mu_{\alpha}
\end{aligned}
$$

where the second line follows from the results of Appendix B. By the same procedure we employed in Appendix B, we find that the cross terms vanish, leaving the direct contribution

$$
T_{\mathrm{n}}=\sum_{\alpha}\left\{\sum_{a \gamma}\left[\mathbf{W}^{\mathrm{T}}\right]_{a \gamma ; \alpha}{ }^{2} / 2 M_{\mathrm{B}}\right\} P_{\alpha}^{2}
$$

Comparing the coefficients of $P_{\alpha}{ }^{2}$ in eqn (C.8) and (C.9), we conclude that

$$
\sum_{a \gamma}\left[\mathbf{W}^{\mathrm{T}}\right]_{a \gamma, \alpha}{ }^{2}=\frac{M_{\mathrm{B}}}{\mu_{\alpha}}
$$

Eqn (C.2) follows immediately from eqn (C.6) and (C.10).

In the specific case where $a \gamma=3 x$ and $\alpha=1,\left[\tilde{\mathbf{W}}^{\mathrm{T}}\right]_{a \gamma ; \alpha}=$ $\left[\tilde{\mathbf{W}}^{\mathrm{T}}\right]_{3 x ; 1}=1$ (see eqn (100)). Therefore the proportionality constant in eqn (106) is $c=1$.

\section{Appendix D}

In eqn (106), $\left\langle j_{3 x}\left(X_{3}=X, t\right)\right\rangle$ is the flux density of (exemplarily) nucleus 3 in the $x$-direction at the point of observation $X_{3}=X$ in the NCM frame; $\left\langle j_{1}\left(Q_{1}=Q=X /\left[\tilde{\mathbf{W}}^{-1}\right]_{3 x ; 1}, t\right)\right\rangle$ is the flux density in the direction of the internal coordinate $Q_{1}$ at the corresponding point of observation $Q_{1}=Q=X /\left[\tilde{\mathbf{W}}^{-1}\right]_{3 x ; 1}$ in the internal frame. Now instead of evaluating the constant of proportionality

$$
c \equiv \frac{\mu_{1}}{M_{3}} \frac{\left[\tilde{\mathbf{W}}^{\mathrm{T}}\right]_{3 x ; 1}}{\left|\left[\tilde{\mathbf{W}}^{-1}\right]_{3 x ; 1}\right|}
$$

in eqn (106) directly, which requires the considerable effort of inverting the matrix $\tilde{\mathbf{W}}$, we infer that it must equal unity through the following rationale. Note that the sign of $c$ is determined by the sign of $\left[\tilde{\mathbf{W}}^{\mathrm{T}}\right]_{3 x ; 1}$ (see Appendix $\mathrm{C}$ ), which is positive.

We assume that the nuclei remain sufficiently well localized that they can be treated classically. Hence, the flux densities are given by

$$
\begin{aligned}
& j_{3 x}(X, t)=\delta\left[X-X_{3}(t)\right] \dot{X}_{3}(t) \\
& j_{1}(Q, t)=\delta\left[Q-Q_{1}(t)\right] \dot{Q}_{1}(t)
\end{aligned}
$$

where $X_{3}(t)$ and $\dot{X}_{3}(t)$ are the position and velocity of nucleus 3 in the NCM frame and $Q_{1}(t)$ and $\dot{Q}_{1}(t)$ are the respective counterparts in the internal frame. During the time interval $\left[t_{1}, t_{2}\right]$ the coordinates $X_{3}$ and $Q_{1}$ traverse the space intervals $\left[X_{3}\left(t_{1}\right), X_{3}\left(t_{2}\right)\right]$ and $\left[Q_{1}\left(t_{1}\right), Q_{1}\left(t_{2}\right)\right]$. Every $X_{3}(t)$ in $\left[X_{3}\left(t_{1}\right), X_{3}\left(t_{2}\right)\right]$ maps into a $Q_{1}(t)$ in $\left[Q_{1}\left(t_{1}\right), Q_{1}\left(t_{2}\right)\right]$ during this time interval, according to the transformation

$$
Q_{1}=X_{3} /\left[\tilde{\mathbf{W}}^{-1}\right]_{3 x ; 1}
$$


Likewise, if the point of observation $X$ lies in $\left[X_{3}\left(t_{1}\right), X_{3}\left(t_{2}\right)\right]$, then the corresponding point of observation $Q$ lies in $\left[Q_{1}\left(t_{1}\right)\right.$, $\left.Q_{1}\left(t_{2}\right)\right]$.

The classical ensemble average of the mean flux density at point of observation $X$ in the NCM frame during time interval $\Delta t=t_{2}-t_{1}$ can be expressed as

$$
\bar{j}_{3 x}(X, \Delta t)=\frac{1}{\mathbb{N} \Delta t} \sum_{i=1}^{\mathbb{N}} \int_{t_{1}}^{t_{2}} \mathrm{~d} t \delta\left[X-X_{3}^{(i)}(t)\right] \dot{X}_{3}^{(i)}(t)
$$

and its internal counterpart as

$$
\bar{j}_{1}(Q, \Delta t)=\frac{1}{\mathbb{N} \Delta t} \sum_{i=1}^{\mathbb{N}} \int_{t_{1}}^{t_{2}} \mathrm{~d} t \delta\left[Q-Q_{1}^{(i)}(t)\right] \dot{Q}_{1}^{(i)}(t)
$$

where $\mathbb{N}$ is the number of members (trajectories) of the ensemble, which are labelled by index $i$. Thus, $X_{3}^{(i)}(t)\left[Q_{1}^{(i)}(t)\right]$ defines the trajectory of the $i$ th member, which is specified by the initial phase $\left(X_{3}^{(i)}(0), \dot{X}_{3}^{(i)}(0)\right)\left[\left(Q_{1}^{(i)}(0), \dot{Q}_{1}^{(i)}(0)\right)\right]$. Changing variables from $t$ to $X_{3}^{(i)}$ in eqn (D.4a) and from $t$ to $Q_{1}^{(i)}$ in eqn (D.4b), we get

$$
\begin{aligned}
& \bar{j}_{3 x}(X, \Delta t)=\frac{1}{\mathbb{N} \Delta t} \sum_{i=1}^{\mathbb{N}} \int_{X_{3}^{(i)}\left(t_{1}\right)}^{X_{3}^{(i)}\left(t_{2}\right)} \mathrm{d} X_{3}{ }^{(i)} \delta\left[X-X_{3}^{(i)}\right] \\
& \bar{j}_{1}(Q, \Delta t)=\frac{1}{\mathbb{N} \Delta t} \sum_{i=1}^{\mathbb{N}} \int_{Q_{1}^{(i)}\left(t_{1}\right)}^{Q_{1}^{(i)}\left(t_{2}\right)} \mathrm{d} Q_{1}{ }^{(i)} \delta\left[Q-Q_{1}^{(i)}\right]
\end{aligned}
$$

For a given trajectory $i$ the integration in eqn (D.5a) yields

$$
\begin{aligned}
& \int_{X_{3}^{(i)}\left(t_{1}\right)}^{X_{3}^{(i)}\left(t_{2}\right)} \mathrm{d} X_{3}^{(i)} \delta\left[X-X_{3}^{(i)}\right] \\
& \quad= \begin{cases}0, & \text { if } X \text { is outside the interval }\left[X_{3}^{(i)}\left(t_{1}\right), X_{3}^{(i)}\left(t_{2}\right)\right] \\
1, & \text { if } X_{3}^{(i)}\left(t_{1}\right)<X<X_{3}^{(i)}\left(t_{2}\right) \\
-1, & \text { if } X_{3}^{(i)}\left(t_{1}\right)>X>X_{3}^{(i)}\left(t_{2}\right)\end{cases}
\end{aligned}
$$

Because $\left[\tilde{\mathbf{W}}^{-1}\right]_{3 x ; 1}>0$, the following conditions hold: if $X_{3}\left(t_{2}\right)>X_{3}\left(t_{1}\right)$, then $Q_{1}\left(t_{2}\right)>Q_{1}\left(t_{1}\right)$; if $X_{3}\left(t_{2}\right)<X_{3}\left(t_{1}\right)$, then $Q_{1}\left(t_{2}\right)<Q_{1}\left(t_{1}\right)$. Thus, the integration in eqn (D.5b) gives

$$
\begin{aligned}
& \int_{Q_{1}^{(i)}\left(t_{1}\right)}^{Q_{1}^{(i)}\left(t_{2}\right)} \mathrm{d} Q_{1}^{(i)} \delta\left[Q-Q_{1}^{(i)}\right] \\
& \quad= \begin{cases}0, & \text { if } Q \text { is outside the interval }\left[Q_{1}^{(i)}\left(t_{1}\right), Q_{1}^{(i)}\left(t_{2}\right)\right] \\
1, & \text { if } Q_{1}^{(i)}\left(t_{1}\right)<Q<Q_{1}^{(i)}\left(t_{2}\right) \\
-1, & \text { if } Q_{1}^{(i)}\left(t_{1}\right)>Q>Q_{1}^{(i)}\left(t_{2}\right)\end{cases}
\end{aligned}
$$

From eqn (D.4), (D.5) and (D.6) it follows that

$$
\bar{j}_{1}(Q, \Delta t)=\bar{j}_{3 x}(X, \Delta t)
$$

In the limit $\Delta t \rightarrow 0$ we identify the mean values $\bar{j}_{3 x}(X, \Delta t)$ and $\bar{j}_{1}(Q, \Delta t)$ with the flux densities $\left\langle j_{3 x}\left(X_{3}=X, t\right)\right\rangle$ and

$$
\begin{aligned}
& \left\langle j_{1}\left(Q_{1}=Q=X /\left[\tilde{\mathbf{W}}^{-1}\right]_{3 x ; 1}, t\right)\right\rangle . \text { That is } \\
& \begin{aligned}
\left\langle j_{3 x}(X, t)\right\rangle & =\lim _{\Delta t \rightarrow 0} \bar{j}_{3 x}(X, \Delta t) \\
& =\lim _{\Delta t \rightarrow 0} \bar{j}_{1}(Q, \Delta t)=\left\langle j_{1}\left(X /\left[\tilde{\mathbf{W}}^{-1}\right]_{3 x ; 1}, t\right)\right\rangle
\end{aligned}
\end{aligned}
$$

We conclude from eqn (106) and (D.8) that $c=1$.

If, on the other hand, it were the case that $\left[\tilde{\mathbf{W}}^{-1}\right]_{3 x ; 1}<0$, the following conditions would hold: if $X_{3}\left(t_{2}\right)>X_{3}\left(t_{1}\right)$, then $Q_{1}\left(t_{2}\right)<Q_{1}\left(t_{1}\right)$; if $X_{3}\left(t_{2}\right)<X_{3}\left(t_{1}\right)$, then $Q_{1}\left(t_{2}\right)>Q_{1}\left(t_{1}\right)$. In this case the integration in eqn (D.5b) would yield

$$
\begin{aligned}
& \int_{Q_{1}^{(i)}\left(t_{1}\right)}^{Q_{1}^{(i)}\left(t_{2}\right)} \mathrm{d} Q_{1}^{(i)} \delta\left[Q-Q_{1}^{(i)}\right] \\
& \quad= \begin{cases}0, & \text { if } Q \text { is outside the interval }\left[Q_{1}^{(i)}\left(t_{1}\right), Q_{1}^{(i)}\left(t_{2}\right)\right] \\
-1, & \text { if } Q_{1}^{(i)}\left(t_{1}\right)<Q<Q_{1}^{(i)}\left(t_{2}\right) \\
1, & \text { if } Q_{1}^{(i)}\left(t_{1}\right)>Q>Q_{1}^{(i)}\left(t_{2}\right)\end{cases}
\end{aligned}
$$

Then we would deduce from eqn (D.4), (D.5), (D.6a) and (D.9) that

$$
\bar{j}_{1}(Q, \Delta t)=-\bar{j}_{3 x}(X, \Delta t)
$$

and therefore, following the argument above that leads to eqn (D.8), that $c=-1$.

\section{Acknowledgements}

We are grateful to Dr T. Grohmann (Evanston) for advice on the molecular symmetry of $\mathrm{B}_{4}$, and to Prof. $\mathrm{H}$. Köppel (Heidelberg), Prof. O. Kühn (Rostock), Priv.-Doz. M. Leibscher (Hannover) and Prof. D. Neumark (Berkeley) for advice on the literature. This work was supported by the Deutsche Forschungsgemeinschaft (projects Ma 515/27-1 and Pe 2297/1-1), the 973 Program of China (2012CB921603), the National Natural Science Foundation of China (grants no. 21373130, 21273140, 21573138, 61378015, 11434007, and 11004125), the Talent Program of Shanxi, the National Science Foundation of Shanxi, China (grant 2014021004), the Program for Chang Jiang Scholars and Innovative Research Teams (grant no. IRT13076), the Shanxi International Cooperation (project 2013081018), the Program for the Innovative Talents of Higher Learning Institution of Shanxi, and the State Key Laboratory of Quantum Optics and Quantum Optics Devices (project KF201402). H.-J. Z. gratefully acknowledges the start-up fund from Shanxi University. J. F. Pérez-Torres thanks the NorthGerman Supercomputing Alliance (HLRN) for a generous allocation of computer time.

\section{References}

1 I. Barth, H.-C. Hege, H. Ikeda, A. Kenfack, M. Koppitz, J. Manz, F. Marquardt and G. K. Paramonov, Chem. Phys. Lett., 2009, 481, 118.

2 E. Schrödinger, Ann. Phys., 1926, 81, 109. 
3 D. R. Herschbach, Angew. Chem., Int. Ed., 1987, 26, 1221.

4 Y. T. Lee, Angew. Chem., Int. Ed., 1987, 26, 939.

5 P. Lazzeretti, Prog. Nucl. Magn. Reson. Spectrosc., 2000, 36, 1.

6 E. Steiner and P. Fowler, J. Phys. Chem. A, 2001, 105, 9553.

7 T. Heine, C. Corminboeuf and G. Seifert, Chem. Rev., 2005, $105,3889$.

8 J. Manz, J. F. Pérez-Torres and Y. Yang, Phys. Rev. Lett., 2013, 111, 153004.

9 E. A. McCullough and R. E. Wyatt, J. Chem. Phys., 1971, 54, 3578.

10 W. H. Miller, J. Chem. Phys., 1974, 61, 1823.

11 W. H. Miller, Acts. Chem. Res., 1993, 26, 174.

12 W. H. Miller, J. Phys. Chem. A, 1998, 102, 793.

13 U. Manthe, T. Seideman and W. H. Miller, J. Chem. Phys., 1994, 101, 4759.

14 T. Wu, H.-J. Werner and U. Manthe, Science, 2004, 306, 2227.

15 A. Accardi, I. Barth, O. Kühn and J. Manz, J. Phys. Chem. A, 2010, 114, 11252.

16 I. Barth, C. Bressler, S. Koseki and J. Manz, Chem. - Asian J., 2012, 7, 1261.

17 T. Bredtmann, H. Katsuki, J. Manz, K. Ohmori and C. Stemmle, Mol. Phys., 2013, 111, 1691.

18 T. Bredtmann, H. Kono, J. Manz, K. Nakamura and C. Stemmle, ChemPhysChem, 2013, 14, 1397.

19 T. Grohmann, J. Manz and A. Schild, Mol. Phys., 2013, 111, 2251.

20 J. Manz, A. Schild, B. Schmidt and Y. Yang, Chem. Phys., 2014, 442, 9.

21 J. F. Pérez-Torres, Phys. Rev. A: At., Mol., Opt. Phys., 2015, 91, 022510.

22 I. Barth, C. Daniel, E. Gindensperger, J. Manz, J. F. PérezTorres, A. Schild, C. Stemmle, D. Sulzer and Y. Yang, in Advances in Multi-Photon Processes and Spectroscopy, ed. S. H. Lin, A. A. Villaeys and Y. Fujimura, World Scientific, New Jersey, 2015, vol. 22, p. 59.

23 I. Barth and J. Manz, Angew. Chem., Int. Ed., 2006, 45, 2962.

24 I. Barth, J. Manz, Y. Shigeta and K. Yagi, J. Am. Chem. Soc., 2006, 128, 7043.

25 I. Barth and J. Manz, in Progress in Ultrafast Intense Laser Science VI, ed. K. Yamanouchi, G. Gerber and A. D. Bandrauk, Springer Verlag, Berlin, 2010, ch. 2, p. 21.

26 H.-C. Hege, J. Manz, F. Marquardt, B. Paulus and A. Schild, Chem. Phys., 2010, 376, 46.

27 T. Bredtmann and J. Manz, Angew. Chem., Int. Ed., 2011, 50, 12652.

28 J. Manz and K. Yamamoto, Mol. Phys., 2012, 110, 517.

29 T. Bredtmann, E. Hupf and B. Paulus, Phys. Chem. Chem. Phys., 2012, 14, 15494.

30 P. von den Hoff, I. Znakovskaya, S. Zherebtsov, M. F. Kling and R. de Vivie-Riedle, Appl. Phys. B, 2010, 98, 659.

31 M. Okuyama and K. Takatsuka K, Chem. Phys. Lett., 2009, 476, 109.

32 K. Nagashima and K. Takatsuka, J. Phys. Chem. A, 2009, 112, 15240.
33 K. Takatsuka and T. Yonehara, Adv. Chem. Phys., 2009, 144, 93.

34 K. Takatsuka and T. Yonehara, Phys. Chem. Chem. Phys, 2011, 13, 4987.

35 M. Okuyama and K. Takatsuka, Bull. Chem. Soc. Jpn., 2012, 86, 217.

36 K. Nagashima and K. Takatsuka, J. Phys. Chem. A, 2012, 116, 11167.

37 S. Patchkovskii, J. Chem. Phys., 2012, 137, 084109.

38 K. Takatsuka, T. Yonehara, K. Hanasaki and Y. Arasaki, Chemical Theory Beyond the Born-Oppenheimer Paradigm: Nonadiabatic Electronic and Nuclear Dynamics in Chemical Reactions, World Scientific, New Jersey, 2015.

39 A. Nitzan and M. A. Ratner, Science, 2003, 300, 1384.

40 Current-Driven Phenomena in Nanoelectronics, ed. T. Seideman, Pan Stanford Publishing, Singapore, 2010.

41 G. C. Solomon, C. Herrmann and M. A. Ratner, Top. Curr. Chem., 2012, 313, 1.

42 A. Kenfack, F. Marquardt, G. K. Paramonov, I. Barth, C. Lasser and B. Paulus, Phys. Rev. A: At., Mol., Opt. Phys., 2010, 81, 052502.

43 A. Kenfack, I. Barth, F. Marquardt and B. Paulus, Phys. Rev. A: At., Mol., Opt. Phys., 2010, 82, 062502.

44 D. Andrae, I. Barth, T. Bredtmann, H.-C. Hege, J. Manz, F. Marquardt and B. Paulus, J. Phys. Chem. B, 2011, 115, 5476.

45 A. Kenfack, S. Banerjee and B. Paulus, Phys. Rev. A: At., Mol., Opt. Phys., 2012, 85, 032501.

46 D. J. Diestler, J. Phys. Chem. A, 2012, 116, 2728.

47 D. J. Diestler, A. Kenfack, J. Manz and B. Paulus, J. Phys. Chem. A, 2012, 116, 2736.

48 D. J. Diestler, J. Phys. Chem. A, 2012, 116, 11161.

49 D. J. Diestler, J. Phys. Chem. A, 2012, 117, 4698.

50 J. F. Pérez-Torres, Phys. Rev. A: At., Mol., Opt. Phys., 2013, 87, 062512.

51 D. J. Diestler, A. Kenfack, J. Manz, B. Paulus, J. F. PérezTorres and V. Pohl, J. Phys. Chem. A, 2013, 117, 8519.

52 J. Manz, J. F. Pérez-Torres and Y. Yang, J. Phys. Chem. A, 2014, 118, 8411.

53 J. F. Pérez-Torres, J. Phys. Chem. A, 2015, 119, 2895.

54 G. Hermann, B. Paulus, J. F. Pérez-Torres and V. Pohl, Phys. Rev. A: At., Mol., Opt. Phys., 2014, 89, 052504.

55 L. A. Nafie, J. Phys. Chem. A, 1997, 101, 7826.

56 T. B. Friedman, X. Gao, M.-L. Shih and L. A. Nafie, J. Phys. Chem. A, 1998, 102, 3352.

57 J. O. Hirschfelder and J. S. Dahler, Proc. Natl. Acad. Sci. U. S. A., 1956, 42, 363.

58 D. W. Jepsen and J. O. Hirschfelder, Proc. Natl. Acad. Sci. U. S. A., 1959, 45, 249.

59 J. O. Hirschfelder, Int. J. Quantum Chem., 1969, 3, 17.

60 B. Friedrich and D. Herschbach, Phys. Rev. Lett., 1995, 74, 4623.

61 H. Stapelfeldt and T. Seideman, Rev. Mod. Phys., 2003, 75, 534.

62 M. Leibscher, I. S. Averbukh and H. Rabitz, Phys. Rev. Lett., 2003, 90, 213001. 
63 R. De Nada, E. Heesel, M. Lein, N. Hay, R. Velotta, E. Spingate, M. Castillejo and J. P. Marangos, Phys. Rev. A: At., Mol., Opt. Phys., 2004, 69, 031804.

64 S. Fleischer, I. S. Averbukh and Y. Prior, Phys. Rev. Lett., 2007, 99, 093002.

65 T. Grohmann and M. Leibscher, J. Chem. Phys., 2011, 134, 204316.

66 F. Filsinger, J. Küpper, G. Meijer, L. Holmegaard, J. H. Nielsen, I. N. Iftach, J. L. Hansen and H. Stapelfeldt, J. Chem. Phys., 2009, 131, 064309.

67 S. Fleischer, Y. Zhou, R. W. Field and K. A. Nelson, Phys. Rev. Lett., 2011, 107, 163603.

68 M. Spanner, S. Patchkovskii, E. Frumker and P. Corkum, Phys. Rev. Lett., 2012, 109, 11300.

69 J. R. Taylor, Scattering Theory: The Quantum Theory on Nonrelativistic Collisions, Wiley, New York, 1972.

70 I. Barth, J. Phys. Chem. A, 2012, 116, 11283.

71 N. E. Henriksen and F. Y. Hansen, Theories of Molecular Reaction Dynamics: The Microscopic Foundation of Chemical Kinetics, Oxford University Press, New York, 2008.

72 P. C. Morse and H. Feshbach, Methods of Theoretical Physics, McGraw-Hill, New York, 1953.

73 F. Hund, Z. Phys., 1927, 43, 805.

74 N. Makri and W. H. Miller, J. Chem. Phys., 1989, 91, 4026.

75 K. Giese, H. Ushiyama and O. Kühn, Chem. Phys. Lett., 2003, 371, 681.

76 G. V. Mil'nikov and H. Nakamura, J. Chem. Phys., 2005, 122, 124311.

77 G. V. Mil'nikov, O. Kühn and H. Nakamura, J. Chem. Phys., 2005, 123, 074308.

78 K. Giese, M. Petković, H. Naundorf and O. Kühn, Phys. Rep., 2006, 430, 211.

79 J. Kästner, Wiley Interdiscip. Rev.: Comput. Mol. Sci., 2014, 4, 158.

80 M. Born and R. Oppenheimer, Ann. Phys., 1927, 84, 457.

81 M. Born and K. Huang, Dynamical Theory of Crystal Lattices, Oxford University Press, London, 1954, Appendix VIII, p. 406.

82 P. Gölitz, E. Wessel, private communication, Weinheim, 2015.

83 T. Niederhausen and U. Thumm, Phys. Rev. A: At., Mol., Opt. Phys., 2008, 77, 013407.

84 A. Rudenko, T. Ergler, B. Feuerstein, K. Zrost, C. D. Schröter, R. Moshammer and J. Ullrich, Chem. Phys., 2006, 329, 193.

85 D. J. Tannor, Introduction to Quantum Mechanics: A Time-Dependent Perspective, University Science Books, Sausalito, 2005.

86 B. Feuerstein, T. Ergler, A. Rudenko, K. Zrost, C. D. Schröter, R. Moshammer and J. Ullrich, Phys. Rev. Lett., 2007, 99, 153002.

87 J. J. Vrakking, D. M. Villeneuve and A. Stolow, Phys. Rev. A: At., Mol., Opt. Phys., 1996, 54, R37.

88 B. Feuerstein and U. Thumm, J. Phys. B: At., Mol. Opt. Phys., 2003, 36, 707.

89 D. Ley, D. Gerbig and P. R. Schreiner, Org. Biomol. Chem., 2012, 10, 3781.
90 S. Albert, P. Lerch, R. Prentner and M. Quack, Angew. Chem., Int. Ed., 2013, 52, 346 (Angew. Chem., 2013, 125, 364).

91 J. M. L. Martin, J. P. Francois and R. Gijbels, Chem. Phys. Lett., 1992, 189, 529.

92 H. J. Zhai, L. S. Wang, A. N. Alexandrova, A. I. Boldyrev and V. G. Zakrzewski, J. Phys. Chem. A, 2003, 107, 9319.

93 R. Linguerri, I. Navizet, P. Rosmus, S. Carter and J. P. Maier, J. Chem. Phys., 2005, 122, 034301.

94 H. J. Zhai, B. Kiran, J. Li and L. S. Wang, Nat. Mater., 2003, 2, 827.

95 B. Kiran, S. Bulusu, H.-J. Zhai, S. Yoo, X. C. Zeng and L.-S. Wang, Proc. Natl. Acad. Sci. U. S. A., 2005, 102, 961.

96 N. G. Szwacki, A. Sadrzadeh and B. I. Yakobson, Phys. Rev. Lett., 2007, 98, 166804.

97 W. Huang, A. P. Sergeeva, H. J. Zhai, B. B. Averkiev, L. S. Wang and A. I. Boldyrev, Nat. Chem., 2010, 2, 202.

98 A. P. Sergeeva, Z. A. Piazza, C. Romanescu, W. L. Li, A. I. Boldyrev and L. S. Wang, J. Am. Chem. Soc., 2012, 134, 18065.

99 I. A. Popov, Z. A. Piazza, W. L. Li, L.-S. Wang and A. I. Boldyrev, J. Chem. Phys., 2013, 139, 144307.

100 W.-L. Li, Y. F. Zhao, H. S. Lu, J. Li and L. S. Wang, Angew. Chem., Int. Ed., 2014, 53, 5540.

101 Z. A. Piazza, H.-S. Hu, W.-L. Li, Y.-F. Zhao, J. Li and L.-S. Wang, Nat. Commun., 2014, 5, 3113.

102 A. P. Sergeeva, I. A. Popov, Z. A. Piazza, W.-L. Li, C. Romanescu, L.-S. Wang and A. I. Boldyrev, Acc. Chem. Res., 2014, 47, 1349.

103 H. J. Zhai, Y. F. Zhao, W. L. Li, Q. Chen, H. Bai, H. S. Hu, Z. A. Piazza, W. J. Tian, H. G. Lu, Y. B. Wu, Y. W. Mu, G. F. Wei, Z. P. Liu, J. Li, S. D. Li and L. S. Wang, Nat. Chem., 2014, 6, 727.

104 Q. Chen, S.-Y. Zhang, H. Bai, W.-J. Tian, T. Gao, H.-R. Li, C.-Q. Miao, Y.-G. Lu, H.-J. Zhai and S.-D. Li, Angew. Chem., Int. Ed., 2015, 54, 8160 (Angew. Chem., 2015, 127, 8278).

105 D.-Z. Li, Q. Chen, Y. B. Wu, H. G. Lu and S. D. Li, Phys. Chem. Chem. Phys., 2012, 14, 14769.

106 W. L. Li, C. Romanescu, T. Jian and L. S. Wang, J. Am. Chem. Soc., 2012, 134, 13228.

107 H. J. Zhai, Q. Chen, H. Bai, H. G. Lu, W. L. Li and S. D. Li, J. Chem. Phys., 2013, 139, 174301.

108 H. Tang and S. Ismail-Beigi, Phys. Rev. Lett., 2007, 99, 115501.

109 X. B. Yang, Y. Ding and J. Ni, Phys. Rev. B: Condens. Matter Mater. Phys., 2008, 77, 041402(R).

110 H. Tang and S. Ismail-Beigi, Phys. Rev. B: Condens. Matter Mater. Phys., 2010, 82, 115412.

111 X. Wu, J. Dai, Y. Zhao, Z. Zhuo, J. Yang and X. C. Zeng, ACS Nano, 2012, 6, 7443.

112 J. Zhang, A. P. Sergeeva, M. Sparta and A. N. Alexandrova, Angew. Chem., Int. Ed., 2012, 51, 8512 (Angew. Chem., 2012, 124, 8640).

113 J. A. Pople, M. Head-Gordon and K. Raghavachari, J. Chem. Phys., 1987, 87, 5968.

114 R. A. Kendall and T. H. Dunning, Jr. and R.J. Harrison, J. Chem. Phys., 1992, 96, 6796. 
115 J. R. Letelier and C. A. Utreras-Díaz, Spectrochim. Acta, Part A, 1997, 53, 247.

116 W. Gordy and R. L. Cook, Microwave Molecular Spectra, Interscience, New York, 1970.

117 T. Baba, T. Tanaka, I. Morino, K. M. T. Yamada and K. Tanaka, J. Chem. Phys., 1999, 110, 4131.

118 G. von Helden, D. van Heijnsbergen and G. Meijer, J. Phys. Chem. A, 2003, 107, 1671.

119 M. L. Weichman, J. B. Kim and D. M. Neumark, J. Chem. Phys., 2014, 140, 104305.

120 Handbook of High-Resolution Spectroscopy, ed. M. Quack and F. Merkt, Wiley, Chichester, 2011.

121 M. Kanno, H. Kono and Y. Fujimura, Angew. Chem., Int. Ed., 2006, 45, 7995.

122 M. Kanno, H. Kono, Y. Fujimura and S. H. Lin, Phys. Rev. Lett., 2010, 104, 108302.

123 X. Zhang, D. A. Hrovat and W. T. Borden, Org. Lett., 2010, 12, 2798.

124 S. Kozuch, X. Zhang, D. A. Hrovat and W. T. Borden, J. Am. Chem. Soc., 2013, 135, 17274.

125 P. R. Schreiner, H. P. Reisenauer, D. Ley, D. Gerbig, C.-H. Wu and W. D. Allden, Science, 2011, 332, 1300.

126 A. C. Cope and E. M. Hardy, J. Am. Chem. Soc., 1940, 62, 441.

127 H. E. Zimmerman and G. L. Grunewald, J. Am. Chem. Soc., 1966, 88, 183.

128 H. Quast, T. Herkert, A. Witzel, E. M. Peters and H. G. von Schnering, Chem. Ber., 1994, 127, 921.

129 R. V. Williams, in Strained Hydrocarbons, ed. H. Dodziuk, Wiley, Weinheim, 2009, p. 399.

130 R. V. Williams, Eur. J. Org. Chem., 2001, 227.

131 R. V. Williams, Chem. Rev., 2001, 101, 1185.

132 A. K. Cheng, F. A. L. Anet, J. Mioduski and J. Meinwald, J. Am. Chem. Soc., 1974, 96, 2887.

133 K. Bergmann, S. Görtler, J. Manz and H. Quast, J. Am. Chem. Soc., 1993, 115, 1490.

134 H. Quast and M. Seefelder, Angew. Chem., Int. Ed., 1999, 38, 1064.

135 A. C. Goren, D. A. Hrovat, M. Seefelder, H. Quast and W. T. Borden, J. Am. Chem. Soc., 2002, 124, 3469.

136 M. Seefelder, M. Heubes, H. Quast, W. D. Edwards, J. R. Armentrout, R. V. Williams, C. J. Cramer, A. C. Goren, D. A. Hrovat and W. T. Borden, J. Org. Chem., 2005, 70, 3437.

137 H.-D. Martin and T. Urbanek, J. Am. Chem. Soc., 1985, 107, 5532.

138 L. M. Jackman, E. Fernandes, M. Heubes and H. Quast, Eur. J. Org. Chem., 1998, 2209.

139 M. J. S. Dewar, J. Am. Chem. Soc., 1984, 106, 209.

140 R. V. Williams and H. A. Kurtz, J. Chem. Soc., Perkin Trans. 2, 1994, 147.

141 H. Jiao, R. Nagelkerke, H. A. Kurtz, R. V. Williams, W. T. Borden and P. von Ragué Schleyer, J. Am. Chem. Soc., 1997, 119, 5921.

142 V. N. Staroverov and E. R. Davidson, J. Mol. Struct., 2001, 573, 81 .
143 M. Garavelli, F. Bernardi, A. Cembran, O. Castaño, L. M. Frutos, M. Merchán and M. Olivucci, J. Am. Chem. Soc., 2002, 124, 13770.

144 E. C. Brown, D. K. Henze and W. T. Borden, J. Am. Chem. Soc., 2002, 124, 14977.

145 S. S. Shaik and P. C. Hiberty, A Chemist's Guide to Valence Bond Theory, Wiley, Hoboken, NJ, 2008.

146 E. C. Brown, R. F. W. Bader and N. H. Werstiuk, J. Phys. Chem. A, 2009, 113, 5254.

147 D. R. Greve, J. Phys. Org. Chem., 2011, 24, 222.

148 T. Bredtmann and B. Paulus, J. Chem. Theory Comput., 2013, 9, 3026.

149 M. Dohle, J. Manz, G. K. Paramonov and H. Quast, Chem. Phys., 1995, 197, 91.

150 T. Rozgony, A. Bartók-Pártay and A. Stirling, J. Phys. Chem. A, 2010, 114, 1207.

151 M. Dohle, J. Manz and G. K. Paramonov, Ber. Bunsenges. Phys. Chem., 1995, 99, 478.

152 M. V. Korolkov, J. Manz and G. K. Paramonov, Chem. Phys., 1996, 105, 10874.

153 T. Bredtmann and J. Manz, J. Chem. Sci., 2012, 124, 121.

154 G. Desimoni, G. Tacconi, A. Barco and G. P. Pollini, Natural Products Synthesis through Pericyclic Reactions, American Chemical Society, Washington, DC, 1987.

$155 \mathrm{~J}$. A. Berson, in Rearrangements in Ground and Excited States, ed. P. de Mayo, Academic Press, New York, 1980, vol. 1, p. 311.

156 T. H. Lowry and K. S. Richardson, Mechanism and Theory in Organic Chemistry, Harper and Row, New York, 1987.

157 E. V. Anslyn and D. A. Dougherty, Modern Physical Organic Chemistry, University Science Books, Sausalito, 2006.

158 M. B. Smith and J. March, Advanced Organic Chemistry: Reactions, Mechanisms, and Structure, Wiley, Hoboken, NJ, 2007.

159 K. P. C. Vollhardt and N. E. Schore, Organic Chemistry Structure and Function, Freeman, New York, 2007.

160 P. H. Scudder, Qualitative Molecular Orbital Theory and Pericyclic Reactions, Wiley, Hoboken, NJ, 2nd edn, 2013.

161 P. H. Scudder, Electron Flow in Organic Chemistry, Wiley, Hoboken, NJ, 2013.

162 R. G. Wilkins, Kinetics and Mechanism of Reactions of Transition Metal Complexes, Verlag Chemie, Weinheim, 1991.

163 R. B. Jordan, Reaction Mechanisms of Inorganic and Organometalic Systems, Oxford University Press, Oxford, 2007.

164 C. K. Mathews, K. E. van Holde and K. G. Ahern, Biochemistry, Prentice Hall, Upper Saddle River, NJ, 1999.

165 D. L. Nelson and M. M. Cox, Lehninger Principles of Biochemistry, Freeman, New York, 2009.

166 R. B. Woodward and R. Hoffmann, The Conservation of Orbital Symmetry, Verlag Chemie, Weinheim, 1970.

167 K. N. Houk, Y. Lee and J. D. Evanseck, Angew. Chem., Int. Ed., 1992, 31, 682.

168 O. Wiest, D. C. Montiel and K. N. Houk, J. Phys. Chem. A, 1997, 101, 8378.

169 B. K. Carpenter, Angew. Chem., Int. Ed., 1998, 37, 3340. 
170 H.-H. Limbach, in Hydrogen Transfer Reactions, ed. J. T. Hynes, J. P. Klinman, H.-H. Limbach and R. L. Schowen, Wiley-VCH Verlag, Weinheim, 2007, p. 135.

171 P. R. Schreiner, J. P. Wagner, H. P. Reisenauer, D. Gerbig, D. Ley, J. Sarka, A. G. Császár, A. Vaughn and W. D. Allen, J. Am. Chem. Soc., 2015, 137, 7828.

172 H.-J. Werner, Mol. Phys., 1996, 89, 645.

173 H.-J. Werner and P. J. Knowles, J. Chem. Phys., 1988, 89, 5803.

174 P. J. Knowles and H.J. Werner, Chem. Phys. Lett., 1988, 145, 514.

175 S. R. Langhoff and E. R. Davidson, Int. J. Quantum Chem., 1974, 8, 61.

176 A. D. Becke, J. Chem. Phys., 1003, 98, 5648.

177 C. Lee, W. Yang and R. G. Parr, Phys. Rev. B: Condens. Matter Mater. Phys., 1988, 37, 785.

178 J. Pipek and P. G. Mezey, J. Chem. Phys., 1989, 90, 4916.

179 T. Bredtmann, Electron-Nuclear Quantum Fluxes in Adiabatic Chemical Processes, PhD thesis, Freie Universität, Berlin, 2013.
180 C. Liu, J. Manz and Y. Yang, J. Phys. B: At., Mol. Opt. Phys., 2015, 48, 164001.

181 T. Frohnmeyer and T. Baumert, Appl. Phys. B: Lasers Opt., 2000, 71, 259.

182 T. Ergler, A. Rudenko, B. Feuerstein, K. Zrost, C. D. Schröter, R. Moshammer and J. Ullrich, Phys. Rev. Lett., 2006, 97, 193001.

183 E. Goulielmakis, Z.-H. Loh, A. Wirth, R. Santra, N. Rohringer, V. Yakovlev, S. Zherebtsov, T. Pfeifer, A. Azzeers, M. Kling, S. Leone and F. Krausz, Nature, 2010, 466, 739.

184 T. Bredtmann, S. Chelkowski and A. D. Bandrauk, Phys. Rev. A: At., Mol., Opt. Phys., 2011, 84, 021401(R).

185 S. Chelkowki, T. Bredtmann and A. D. Bandrauk, Phys. Rev. A: At., Mol., Opt. Phys., 2012, 85, 033404.

186 H. J. Suominen and A. Kirander, Phys. Rev. Lett., 2014, 112, 043002.

187 T. Bredtmann, G. Dixit and M. Ivanov, Nat. Commun., 2014, 5, 5589 . 